\title{
CHARACTERIZATION OF A VISCOELASTIC RESPONSE FROM THIN METAL FILMS DEPOSITED ON SILICON FOR MICROSYSTEM APPLICATIONS
}

\author{
A Thesis \\ presented to \\ the Faculty of California Polytechnic State University, \\ San Luis Obispo
}

In Partial Fulfillment

of the Requirements for the Degree

Master of Science in Engineering, with Specialization in Materials Engineering

by

Steven Meredith

January 2009 
(C) 2008

Steven Meredith

ALL RIGHTS RESERVED 


\section{COMMITTEE MEMBERSHIP}

TITLE: $\quad$ Characterization of a Viscoelastic Response from Thin Metal Films Deposited on Silicon for Microsystem Applications

AUTHOR: $\quad$ Steven Meredith

DATE SUBMITTED: January 2009

COMMITEE CHAIR: $\quad$ Dr. Richard Savage, Professor, Materials Engineering

COMMITTEE MEMBER: $\quad$ Dr. Blair London, Professor, Materials Engineering

COMMITTEE MEMBER: Dr. Lanny Griffin, Department Chair, Biomedical Engineering 


\begin{abstract}
Characterization of a Viscoelastic Response from Thin Metal Films Deposited on Silicon for Microsystem Applications
\end{abstract}

Steven Meredith

Understanding the mechanisms that control the mechanical behavior of microscale actuators is necessary to design an actuator that responds to an applied actuation force with the desired behavior. Micro actuators which employ a diaphragm supported by torsional hinges which deform during actuation are used in many applications where device stability and reliability are critical. The material response to the stress developed within the hinge during actuation controls how the actuator will respond to the actuating force. A fully recoverable non-linear viscoelastic response has been observed in electrostatically driven micro actuators employing torsional hinges of silicon covered with thin metal films. The viscoelastic response occurs over a time period of 50 minutes at an operating temperature of $35^{\circ} \mathrm{C}$. This viscoelastic phenomenon is similar to that reported in articles addressing anelastic behavior associated with viscous grain boundary slippage and dislocation bowing. In order to investigate this viscoelastic response as a function of metal film composition and thickness, bi-layer torsional hinge actuators consisting of Si with a deposited metal layer were designed to exhibit similar stress levels as the electrostatically driven micro actuators. The test devices were fabricated using common semiconductor fabrication techniques. The actuators were micromachined by deep etching $100 \mathrm{~mm}$ diameter, $425 \mu \mathrm{m}$ thick, double side polished, single crystal (100) wafers to create a $4.5 \mu \mathrm{m}$ thick device layer. Subsequent etching of the device layer released the fixed-fixed torsional hinge test actuators. Physical vapor depositions of $\mathrm{Au}, \mathrm{Al}$ and $\mathrm{Al}-\mathrm{Ti}$ in two different thicknesses $(100 \mathrm{~nm}$, and $150 \mathrm{~nm})$ were deposited in order to investigate the impact of metal film 
thickness and composition on the viscoelastic response. Grain sizes of the deposited films were estimated using backscattered electron images. Rotational actuation of the test actuators was achieved by using a modified Ambios XP-1 surface profiler that applies a constant force of $0.28 \mathrm{mN}$ while measuring the displacement of the actuator with respect to time. The viscoelastic response was observed in the test devices with $\mathrm{Au}$ and $\mathrm{Al}$ thin films indicating that this phenomenon is attributable to the stresses induced on the torsional hinge. Results indicate that the viscoelastic response was not observed in AlTi thin films consisting of $0.3 \mathrm{at} \%$ titanium. Two theoretical models are presented that discuss the mechanism associated with the viscoelastic response as well as a method for inhibiting these mechanisms by the addition of an alloying element to form a second phase precipitate.

Keywords: MEMS, viscoelasticity, thin film, grain boundary diffusion, dislocation bowing 


\section{ACKNOWLEDGMENTS}

My sincere gratitude to the following people and organizations for their support over the past two years:

Brent Huigens for starting this project and providing a constant balance against my skepticism.

My advisor, Dr. Richard Savage for his support and advice in the development of this project as well as my progress throughout my educational experience at Cal Poly.

The Microsystems Technology Group who provided their support and advice throughout this project.

Brian Stahl for his willingness to replace the SEM filaments each time they failed and for keeping the profilometer in working condition.

Olympus Corporate R\&D, Japan for their technical support, financial support over the past two years and providing an exciting engineering project.

Cal Poly Materials Engineering Department for providing the facilities that allowed this project to develop. 


\section{TABLE OF CONTENTS}

LIST OF TABLES …………………………………………………………………. ix

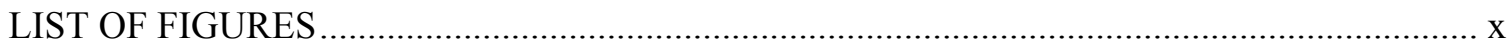

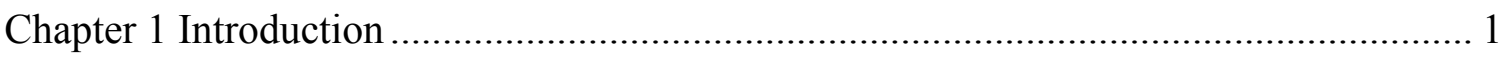

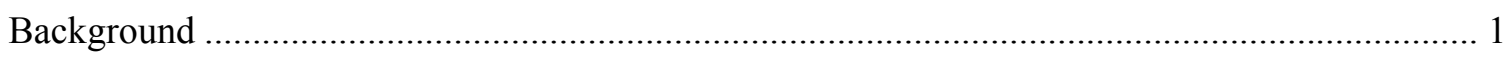

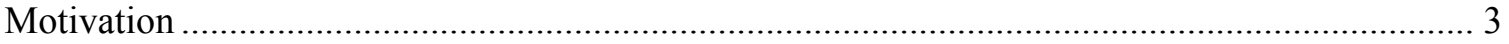

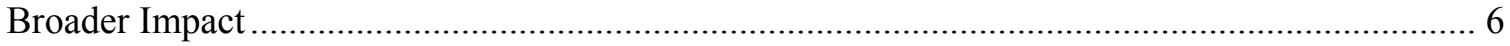

Necessity of Microscale Devices....................................................................................... 7

Chapter 2 Principles of Operation and Design ........................................................... 9

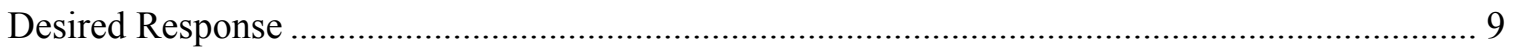

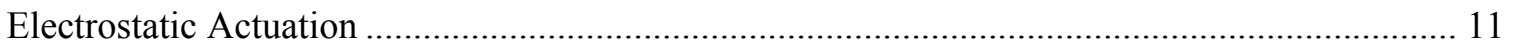

Chapter 3 Testing and Characterization Equipment ........................................................ 16

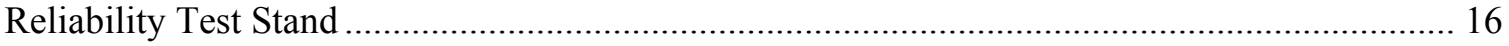

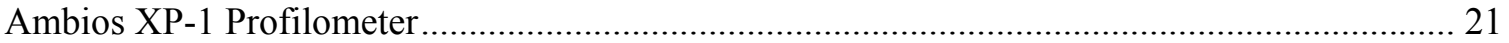

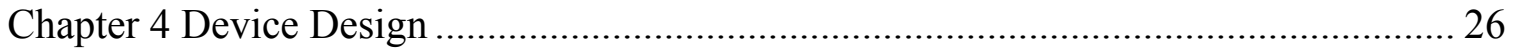

Overview of design and fabrication process ...................................................... 26

Chapter 5 Fabrication of Test Device ………………………................................... 32

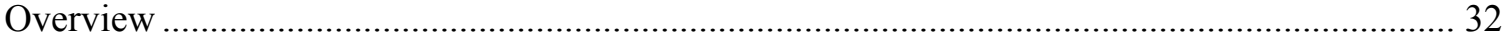

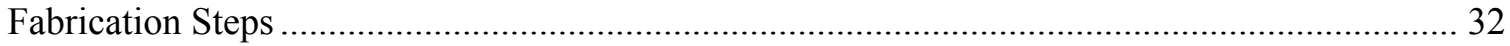

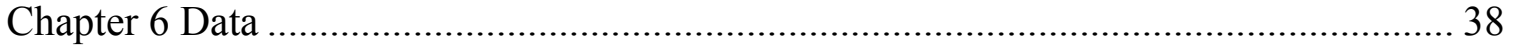

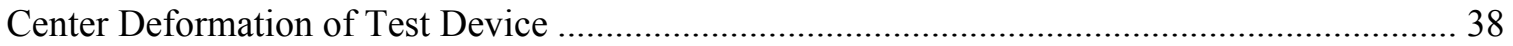

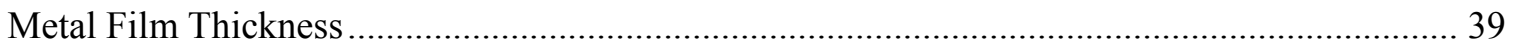

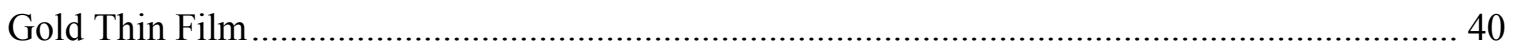

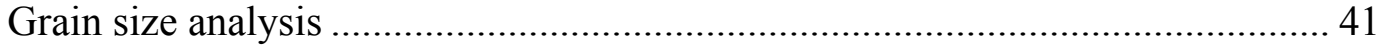

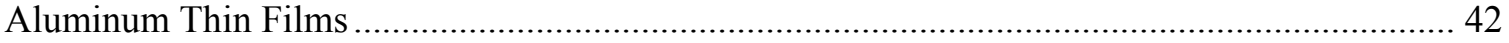

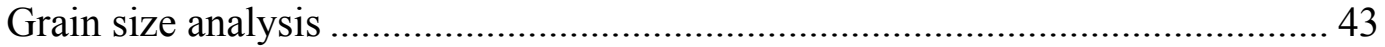

vii 
Chapter 7 Discussion

Viscoelastic Mechanism - Theory ………………………………………………………. 45

Point Defect Diffusion at Grain Boundaries......................................................................... 45

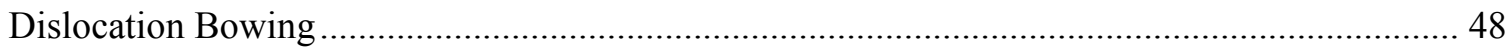

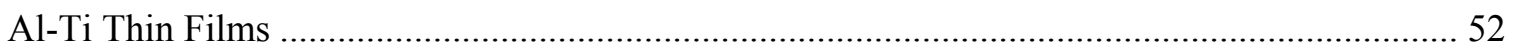

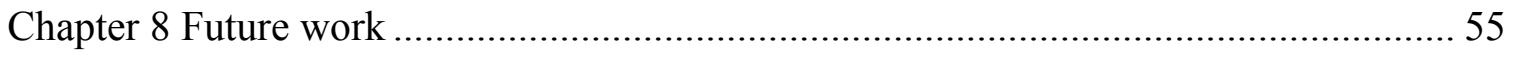

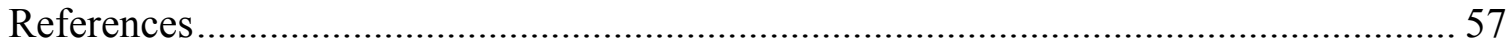

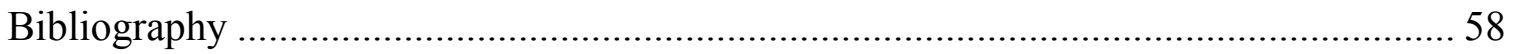

Appendix A. Lithography Masks ........................................................................ A-1

Appendix B. Finite Element Analysis Data............................................................ B-1

Fixed-Fixed torsional hinge test device model ................................................ B-1

Torsional Hinge Spring Constant................................................................. B-3

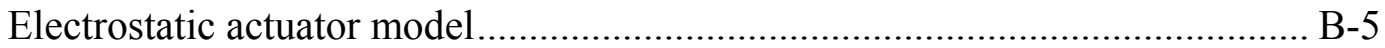

Appendix C. Mechanical Drawings and RTS BOM.................................................. C-1 


\section{LIST OF TABLES}

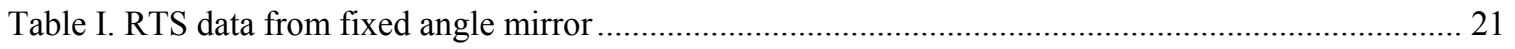

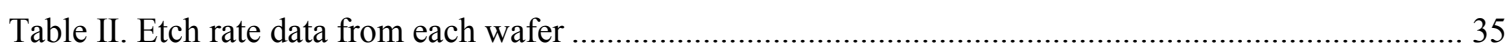

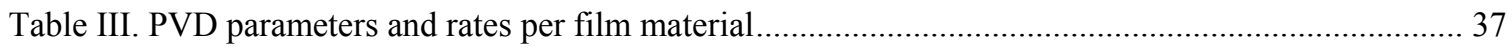

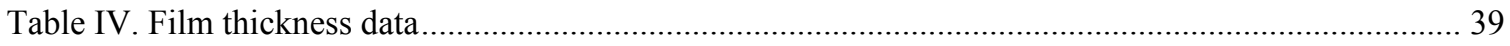

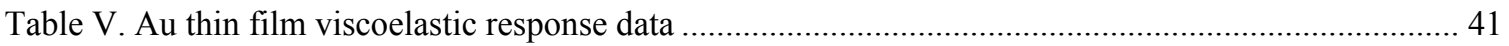

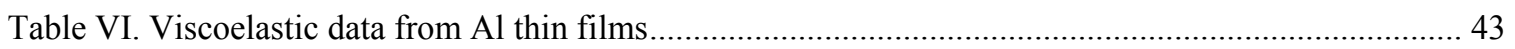

Table VII. Results from Al thin films compared to AlTi thin films .......................................................... 53

Table VIII. Comparison of FEA model to hand calculation and measured values.........................................

Table IX. Data comparison between rotational FEA model and hand calculation ........................................ 


\section{LIST OF FIGURES}

Figure 1. a) Initial fiber alignment and b) electrostatic actuation switch to alternate fiber at output $2 \ldots \ldots . . .5$

Figure 2. Signal loss due to distance traveled through free space ............................................................. 7

Figure 3. The desired linear elastic rotational response of a micro actuator over time ................................ 10

Figure 4. The time dependent viscoelastic response recorded from the electrostatic actuators. a) Linear elastic actuation from $0^{\circ}$ to $-3.2^{\circ}$ b) Viscoelastic response of actuator (linear elastic portion

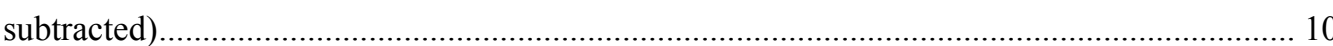

Figure 5. The observed behavior is time dependent where after the initial switch the actuator

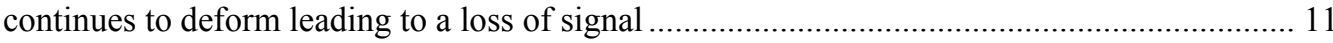

Figure 6. Parallel plate capacitor model for electrostatic actuation........................................................ 12

Figure 7. Electrostatic actuation to induce a rotation about a torsional hinge ........................................... 14

Figure 8. Reliability test stand and accompanying equipment .................................................................... 17

Figure 9. Plan view of RTS indicating the AOI and the perpendicular distance between the actuator

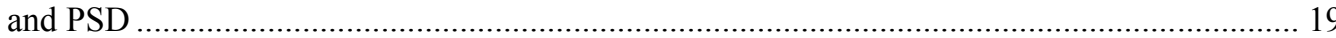

Figure 10. Measurements from fixed angle mirrors verify that the viscoelastic response observed is due to the electrostatic actuators and not a measurement error.

Figure 11. a) Data collected from the fixed angle mirrors verifies that the RTS is not producing the viscoelastic response due to a measurement error b) Detail of $-3.88^{\circ}$ fixed mirror data............. 21

Figure 12. Ambios profilometer viscoelastic measurement process.......................................................... 22

Figure 13. Measured force applied to a milligram scale by the profilometer stylus over an 18 minute time period.

Figure 14. a) Stylus alignment over device b) Engagement of stylus onto edge of device c) Force applied to device to create rotation immediately followed by data logging of deflection.......... 24

Figure 15. Average reading while monitoring a solid silicon wafer, overall average measurement is $4 \pm 20 \mathrm{~nm}$ of drift \pm the background noise of the system.

Figure 16. ABAQUS FEM von Mises stress map indicating the stress distribution through the hinge design, S, Mises scale x $10^{6}$

Figure 17. Test device design utilizing $150 \mu \mathrm{m} \times 40 \mu \mathrm{m}$ torsional hinges with an average device thickness of $4.5 \mu \mathrm{m}$

Figure 18. Top view von Mises stress plot of test device indicating $14 \mathrm{MPa}$ to $20 \mathrm{MPa}$ of stress distributed across both torsional hinges

Figure 19. Isometric view of the test device and hinge area indicating the area where the stress is concentrated 
Figure 20. Test device deformation plots a) Deformation symbol plot (10x) showing minimal bending of the actuator face under torsional loading b) Top view displacement map of test device under torsional load

Figure 21. Distance to neutral axis of rotation

Figure 22. Deformation along center line of device plate showing minimal bending of device plate under torsional load

Figure 23. Average etch rate of all wafers at $85^{\circ} \mathrm{C}$ in $9: 1$ by volume $25 \mathrm{wt} \% \mathrm{TMAH}$ :Isopropyl alcohol with a grand average of $25.90 \pm 0.44 \mu \mathrm{m} / \mathrm{hr}$

Figure 24 a) The red dashed line shows the scan path across the device hinge b) A typical scan profile showing where the maximum deflection is measured ............................................................... 38

Figure 25. Before and after metal layers were applied to the test devices............................................... 39

Figure 26. a) Viscoelastic response of $100 \mathrm{~nm}$ gold film b) Viscoelastic response of $150 \mathrm{~nm}$ gold film...... 40

Figure 27. Comparison of viscoelastic response of the gold film.............................................................. 41

Figure 28. BSE images of the thin gold films indicating grain size is similar between the two films thickness. The dark outlines are added to indicate grain size. a) 100nm Au film b) $150 \mathrm{~nm}$ $\mathrm{Au}$ film

Figure 29. a) Viscoelastic response of the $100 \mathrm{~nm} \mathrm{Al} \mathrm{film} \mathrm{b)} \mathrm{Viscoelastic} \mathrm{response} \mathrm{of} \mathrm{the} 150 \mathrm{~nm} \mathrm{Al}$ film.

Figure 30. Comparison of the $\mathrm{Al}$ film thickness on the amount of viscoelastic response

Figure 31. BSE images of the thin aluminum films indicating grain size is similar between the two films thickness. The dark outlines are added to indicate grain size. a) 100nm Al film b) $150 \mathrm{~nm}$ Al film

Figure 32. Point defects along grain boundaries diffuse to grain triple points under applied stress.......

Figure 33. Surface area to volume ratio for various grain diameters.

Figure 34. Dislocations within grains bow under an applied stress through pipe diffusion along the dislocation core where atomic jumps leave vacancies behind (10).

Figure 35. 100nm Al film compared to the $100 \mathrm{~nm}$ Al-Ti film.

Figure 36. Comparison of the $150 \mathrm{~nm}$ Al film and the $150 \mathrm{~nm}$ Al-Ti film showing a reduction in viscoelastic response.

Figure 37 Mask 1 - Used to create the exposed silicon areas for deep etch wells on the back side of the wafer

Figure 38 Mask 2 applied to top side of wafer to create the

Figure 39. Boundary conditions applied to center deformation test device model.

Figure 40. Boundary condition on torsional test device model

Figure 41. Deformation plot with applied load at center of device of the test device showing mesh density 
Figure 42. Mesh convergence of FEA model with applied force at the center of the device B-3

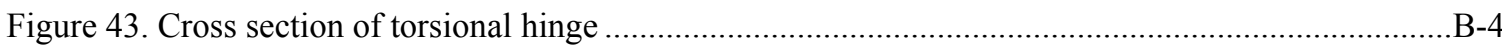

Figure 44. Mesh convergence of FEA model under a torsional load ......................................................

Figure 45. Boundary conditions applied to electrostatic model hinge.....................................................

Figure 46. Mesh convergence for the electrostatic actuator model .......................................................... B-6 


\section{Chapter 1 Introduction}

\section{Background}

Microelectromechanical systems (MEMS) were developed based on the material fabrication processes adapted from the integrated circuit manufacturing industry. As scientists and engineers developed techniques to create integrated circuits, they realized that the same processes could be used to create microscale structures like cantilevers, gears, switches and diaphragms which could be integrated into micro machines. Silicon is the base material for integrated circuits due to the relative ease with which its electronic properties can be modified. As such, fabrication processes such as micromachining (etching), physical vapor deposition (PVD) of metal layers, and many others were developed for the base material of silicon. Accordingly, silicon has become the foundation from which MEMS have been developed. Much like steel creates the structure of many buildings and bridges, silicon creates the structure of microscale devices. It is the scale of these structures that give them their main benefits including sensitivity, cost and reliability. Structures at this scale are more sensitive than their larger macro-scale counterparts due to their size (1). The amount of force required to deform microscale devices is much smaller, in the realm of millinewtons, than that required to deform a macro scale device. Combining this increased mechanical sensitivity with electrostatics that control the devices electrically creates a highly sensitive sensor that requires small amounts of power. Adding to these benefits, the planar fabrication processes developed for integrated circuits and used to create MEMS allow for hundreds to thousands of devices to be created at the same time with high precision and low cost. 
Batch fabrication reduces the cost of micro devices by eliminating the need for assembly of multiple parts to create an equivalent macro-scale device. This equates to consistency between individual devices with respect to mechanical behavior and electrical response. Furthermore, by embedding electrical circuits into the structures, the micro devices can be calibrated with respect to one another by characterizing mechanical behavior variation between the devices as a function of the electrical input. This allows each device to be calibrated through electrical input so that the mechanical response is uniform among all of the devices. Not only are the devices fabricated to yield high uniformity but variation of device performance can be adjusted for through the calibration of the electrical signals controlling the device performance.

There is a limit, however, to the amount of control that can exist for a device. As devices are designed and fabricated at the micro and nano scale, mechanical behavior of the materials used to create the device begin to differ from what has been observed at the macro scale. One such behavior is a time dependent deformation response to an applied force. This type of behavior is referred to as a viscoelastic response where the mechanical response of a device is characterized as having an elastic component combined with a viscous component. Devices with a viscoelastic response will deform elastically to an applied force and then continue to deform with time dependence (viscous) until an equilibrium displacement has been achieved. Upon removal of the applied force, the device will recover the elastic portion of the deformation minus a portion of the viscous deformation. If the total amount of deformation, the elastic and viscous portion, is fully recovered then the behavior can be considered anelastic (2). 
Anelasticity describes the mechanical response of a material when it exhibits a time dependent deformation response to an applied force and a time dependent response to the removal of the force where the deformation of the device is fully recovered (3).

\section{Motivation}

Time dependent behavior is of great concern to microsystem designers and engineers since it can adversely affect the desired response of the device. The time dependent deformation can result in device characteristics that decrease reliability. Understanding the mechanism that is creating this time dependent response is critical in developing methods to reduce and ultimately eliminate this undesired response.

The digital-micro-mirror device (DMD) created by Texas Instruments (TI) is an example of where MEMS has been successfully introduced into the mass market (4). TI uses an array of over 2 million electrostatically actuated mirrors that switch on and off to project an image. The DMD device utilizes a source light reflected from individual micro mirrors which allows for much brighter images to be displayed compared to LCD displays. Each mirror corresponds to one pixel within the image and the color of that pixel can be adjusted by sending the light through a color wheel of red, green and blue. To create a range of colors, the mirrors are switched on and off in a pulsing fashion. For example, to display the color yellow, the mirrors are timed to pulse light from the color wheel for only red and green light. The creation of a grey scale image is created by pulsing light where a longer pulse time results in a brighter pixel and a shorter pulse time will result in a darker pixel. The control of the pulse duration is critical in controlling the 
grey scale of the image. If the pulse duration is not consistent over time, a grey scale image that is being projected will slowly change in contrast. This was an issue that was encountered with the DMD projection system where images projected for extended periods of time slowly changed color and contrast. This was due to a time dependent stress relaxation in the thin aluminum films used to create the individual mirrors and the supporting silicon structure (4). This time dependent stress relaxation changed how the mirrors behaved mechanically over time which, in turn, changed the pulse duration of the individual pixels in the image being projected. A small change in the mechanical behavior due to the time dependent response of a thin film used to create a mirror surface resulted in an unstable image.

Microsystem devices are also being utilized in telecommunication devices such as cell phone antennas, television signals and radio broadcasts as high frequency RF switches. Electrostatically actuated RF switches are attractive because they require low operating power and can be fabricated at low cost using the semiconductor fabrication processes. The switch is created using a thin metal film that spans a distance over an electrode much like a bridge spans a river. To actuate the switch, a voltage is created between the metal film and an electrode inducing an electrostatic force which causes the metal film to deform and come into contact with the electrode. This turns the switch on by using low power electrostatics. To turn the switch off, the voltage is removed and the mechanical force that is stored in the thin film causes it to return to its original position; however, a sticking point has been encountered with this switching approach where the mechanical restoring force within the hinge of the device changes over time. This stress relaxation 
reduces the mechanical force stored in the metal film that is used to release the switch from the electrode, effectively rendering the switch to be stuck in the on position (5).

These two examples show the importance of understanding how materials behave at the micro scale. With both devices, an undesired time dependent stress relaxation mechanism resulted in performance loss or device failure. In both situations the thin films were expected to behave with an instantaneous linear elastic response, however, this was not the case and the device's stability and reliability were compromised.

Another example where the linear elastic response is critical is when switching an optical signal from one fiber optic path to another. Figure 1 depicts a device that rotates about a central hinge to route light using electrostatic actuation to tilt a mirror surface and align the signal from output 1 to output 2 .

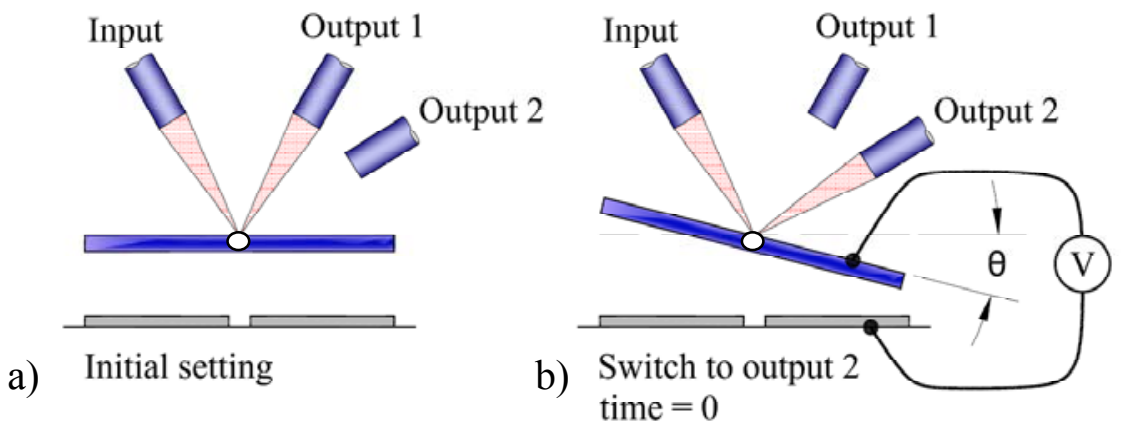

Figure 1. a) Initial fiber alignment and b) electrostatic actuation switch to alternate fiber at output 2.

This type of device could be used in a telecommunications application where an optical signal is transferred from one pathway to another. Current telecommunications pathways are switched through electrical means where the input optical signal is converted into an 
electrical signal. That electrical signal is then routed to the desired output path and then converted back into an optical signal. This conversion process, optical-electrical-optical (OEO), limits the amount of data that can be transferred to 30 gigabytes per second. If the signal was switched using an electrostatic actuator where the signal remains completely optical, the transfer rate, or the amount of information, can be increased by three orders of magnitude to 40 terabytes per second. The completely optical system, however, requires a high degree of stability, $\pm 0.16^{\circ}$ under all conditions, to maintain signal quality.

\section{Broader Impact}

The ability to increase the volume of information that can be sent through an optical pathway would have many social benefits. An all optical network would enable the transfer of software storage from the home computer to a centralized network computer. The typical user would own a much simpler computer that accessed software through the internet. This would shift the responsibility of maintenance, security and upgrades of software and computer components from the end user to a service supplier. The need to mass produce items like hard drives, disk drives, cables, software packaging and a myriad of computer components would no longer be required. Instead, equipment upgrades and component service requirements would be handled at a centralized location much like internet service providers maintain and upgrade the hardware and software required to provide internet access to communities. The end users could access their virtual desktops where all of their software and data would be available from any workstation by simply logging into their service account in a similar fashion as internet e-mail accounts are used 
today. Businesses would benefit by being relieved of the need to constantly update software and components at each workstation for their employees.

\section{Necessity of Microscale Devices}

An additional requirement can only be met by the use of microscale switches. When the optical signal exits the fiber optic path and enters free space, it will begin to degrade as a function of the free space path length. In order to limit the amount of signal degradation, the path the signal travels needs to be minimized. Figure 2 shows how the signal will lose its strength as a function of the distance the signal travels through free space (6).

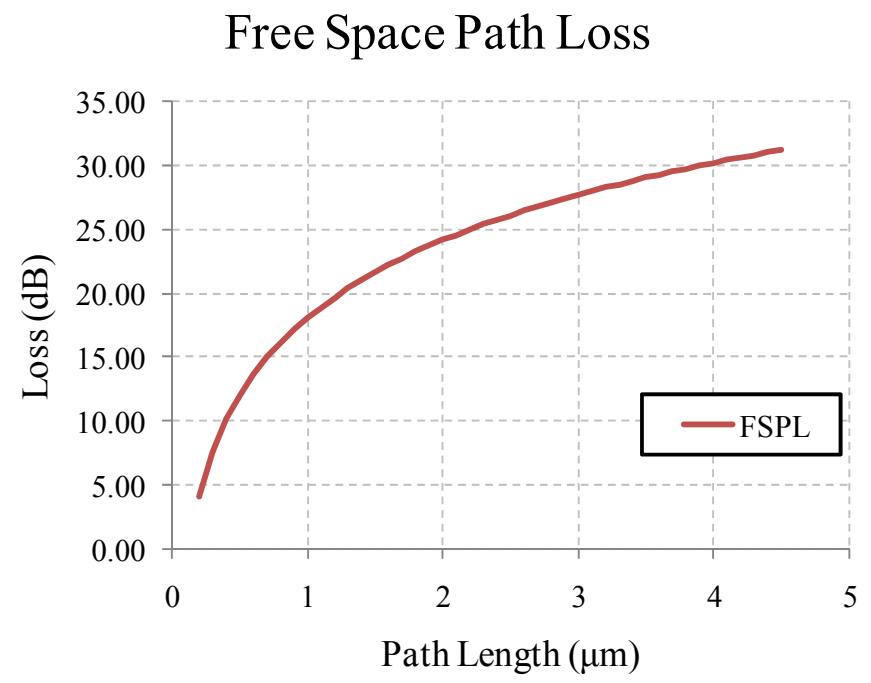

Figure 2. Signal loss due to distance traveled through free space.

In order to minimize the loss due to the path length the light travels, the switches need to be fabricated at the microscale to reduce the path length. Microscale fabrication has the benefit that it allows for multiple signal paths to be closely spaced together giving the system greater flexibility while minimizing the path length loss. As material dimensions are reduced to the micro and nano scale, however, the mechanical behavior of the 
material will change from what has been characterized and tested at the bulk scale leading to the necessity of characterization and testing to verify device behavior. The stability of an all optical network that utilizes electrostatic actuators to route information from one path to another will depend on the ability of the actuator to respond with a linear elastic behavior where the position of the actuator remains constant through time. 


\section{Chapter 2 Principles of Operation and Design}

\section{Desired Response}

An optical switch that is rotationally actuated to align a signal from one fiber to the next requires rotational stability and accuracy in order to maintain signal strength. The precision of the angle is not critical since the actuator can be aligned to each optical output in an initial set-up to maximize signal strength; however, this initial alignment requires that the actuator rotation not drift with respect to time.

In an ideal situation the actuators would behave elastically, deflecting to the desired rotation and holding that position until released. Figure 3 depicts how the ideal actuator would behave when rotated through an actuation of $0^{\circ}$ to $-3.2^{\circ}$ with respect to time. The loading curve in Figure 3 responds in a linear elastic form where an instantaneous deformation occurs when a voltage is applied across the actuator and the actuator rotates to the desired angle and remains at that angle until the voltage is removed. Upon removal of the voltage, the actuator returns to its original position instantly as shown in the unloading curve. 


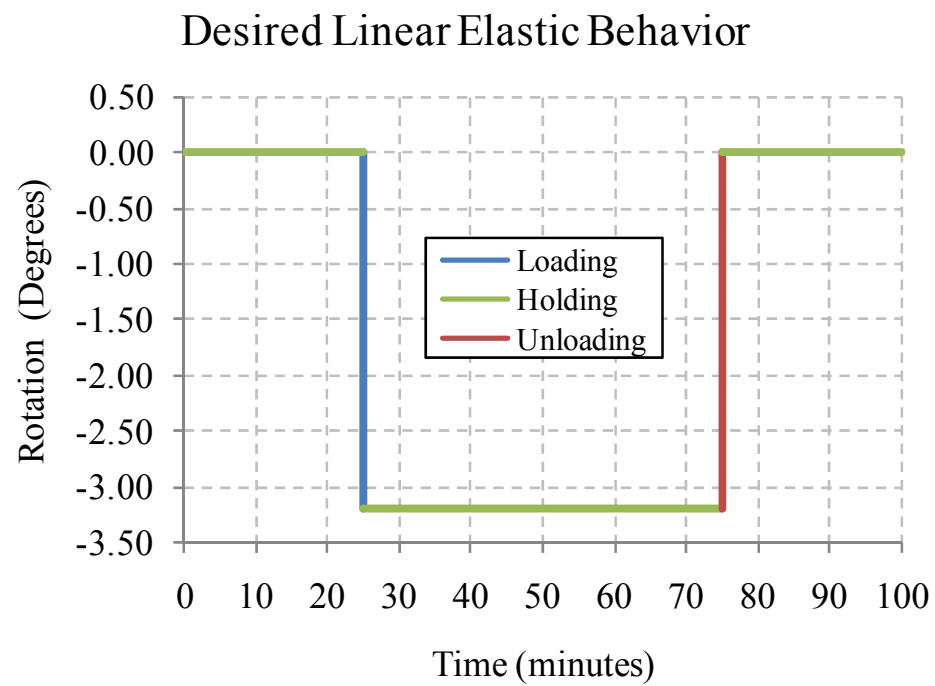

Figure 3. The desired linear elastic rotational response of a micro actuator over time.

Mechanical characterization completed in a previous study revealed a viscoelastic response in micro actuators designed to switch an optical signal (7). Figure 4a presents rotational position data recorded over a 100 minute time period for an actuator for both the unloading (blue) and loading (red) of the actuator. Figure $4 \mathrm{~b}$ shows the viscoelastic portion of the actuation only; the linear elastic rotation of the device was subtracted from this graph in order to highlight the viscoelastic response.

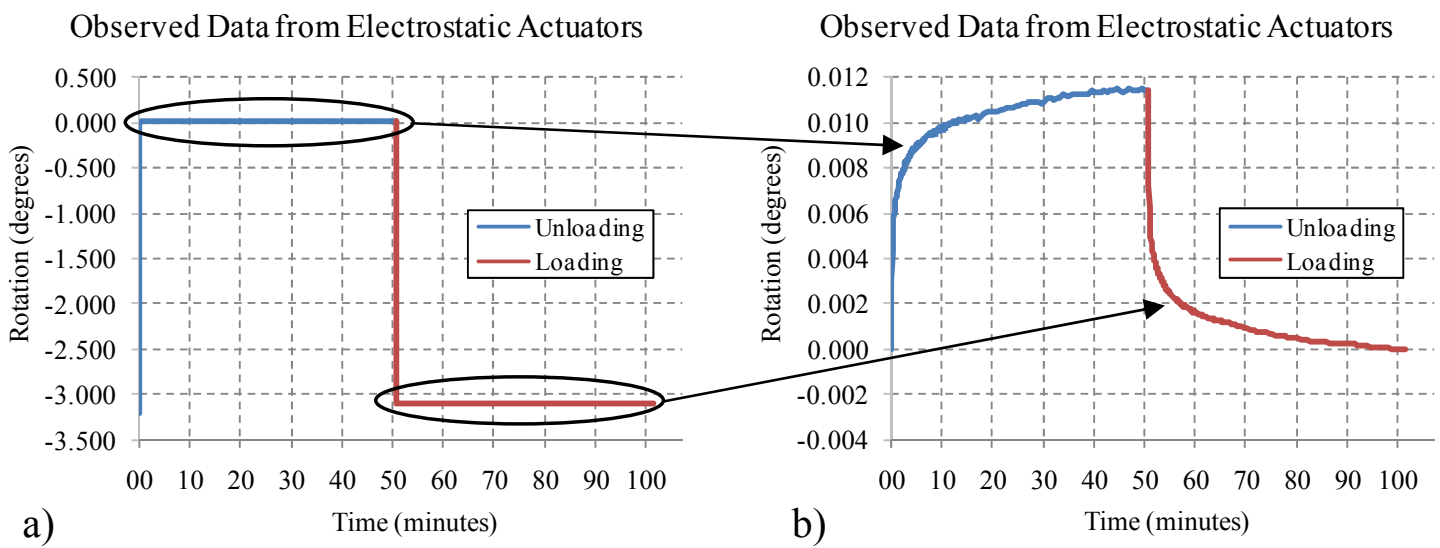

Figure 4. The time dependent viscoelastic response recorded from the electrostatic actuators. a) Linear elastic actuation from $0^{\circ}$ to $-3.2^{\circ}$, b) Viscoelastic response of actuator (linear elastic portion subtracted). 
The observed behavior of the devices differs from the ideal in that they exhibit a time dependent viscoelastic response where the device continues to rotate as a function of time (Figure 5) until after a finite time limit when the device comes to an equilibrium position.

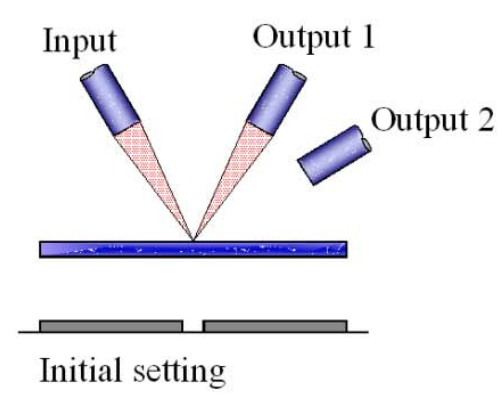

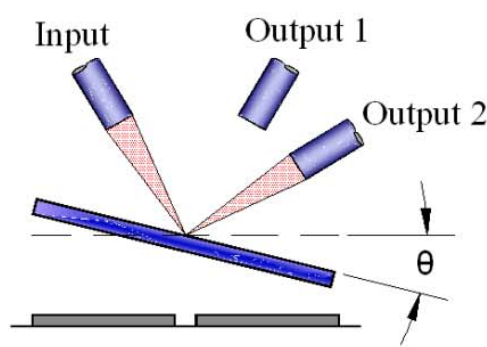

Switch to output 2 time $=0$

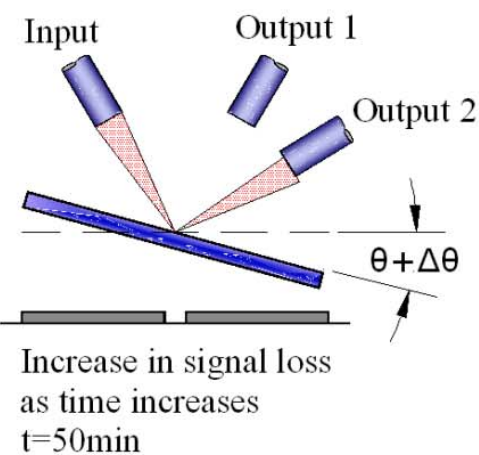

$\mathrm{t}=50 \mathrm{~min}$

Figure 5. The observed behavior is time dependent where after the initial switch the actuator continues to deform $(\Delta \theta)$ leading to a loss of signal.

This time dependent viscoelastic response leads to signal loss as the actuator continues to rotate past its initial alignment position. An ideal actuator would behave in a linear elastic fashion where the actuator rotates to and remains at its initial alignment position. Investigating the causes and magnitude of the viscoelastic response and how to mitigate it is one of the motivations behind this study.

\section{Electrostatic Actuation}

To identify the source for the viscoelastic response ( $\Delta \theta$ in Figure 5), it is convenient to divide the actuating system into two distinct elements: the electrostatic system employed to induce the rotation and the mechanical system that resists the rotation. Electrostatic actuation can be modeled as a parallel plate capacitor where one plate acts as the deformable actuator and the other as a fixed immovable electrode. When a voltage is 
applied across the gap between these two plates an electrostatic force is induced. The actuator is typically constructed of a silicon substrate and a thin metal film. The silicon substrate acts as the structural component that forms the shape of the actuator and resists the electrostatic force. A thin metal film deposited onto the silicon substrate provides the conductive path required to apply the driving voltage. The immovable plate is referred to as the electrode and provides the second conductive path for the driving voltage. Since the electrode is immovable, the electrostatic force developed between the two plates acts to deform the actuator. The amount the actuator deforms for a given amount of applied voltage will depend on the actuator's ability to resist the electrostatic force much like a spring resists a force pulling on it. Figure 6 depicts a parallel plate capacitor model for electrostatic actuation.

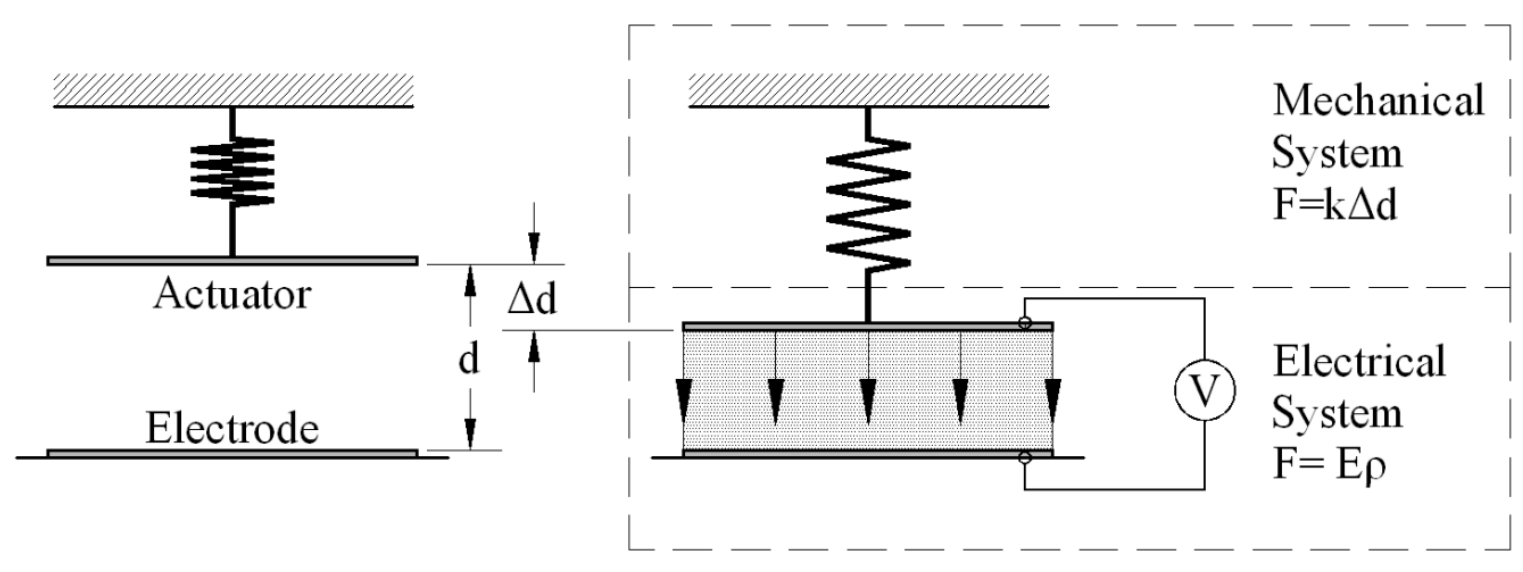

Figure 6. Parallel plate capacitor model for electrostatic actuation.

The actuator in this model is attached to a spring which acts to resist the electrostatic force created between the plates when a voltage is applied. The spring in this illustration represents the part of the actuator, namely the hinge, which deforms under the applied 
electrostatic force. The restoring force $\left(\mathrm{F}_{\text {mech }}\right)$ of the hinge can be calculated using Hooke's law, Equation 1.

$$
F_{\text {mech }}=k \Delta d
$$

Where $\mathrm{F}_{\text {mech }}$ is the restoring mechanical force in Newtons $(\mathrm{N}), \mathrm{k}$ is the spring constant $(\mathrm{N} / \mathrm{m})$ and $\Delta \mathrm{d}$ is the change in distance in $(\mathrm{m})$. The spring constant of the hinge depends on the stiffness of the materials used to create the device as well as the geometry of the hinge. Since the mechanical restoring force is induced as a result of the electrostatic force, the two must balance. The electrostatic force $\left(\mathrm{F}_{\text {elec }}\right)$ for a $\mathrm{DC}$ actuator can be calculated using Equation 2.

$$
F_{\text {elec }}=E_{\text {field }} \times \rho
$$

Where $\mathrm{F}_{\text {elec }}$ is the attractive electrostatic force $(\mathrm{N})$ developed between the actuator and the electrode, $E_{\text {field }}$ is the electric field created between the actuator and electrode and $\rho$ is the charge density between the actuator and electrode.

$$
E_{\text {field }}=\frac{V}{d}
$$

The electric field is dependent on the applied voltage, V, and the distance separating the electrode and actuator, $\mathrm{d}$. The charge density also depends on the applied voltage as well as the capacitance between the actuator and electrode, C, (Eq. 4).

$$
\rho=C V
$$




$$
C=\frac{\varepsilon_{r} \varepsilon_{0} A}{d}
$$

The capacitance depends on the relative permittivity of air, $\varepsilon_{\mathrm{r}}$ (unitless), $\varepsilon_{0}$, the permittivity of free space $\left(8.85 \times 10^{-12} \mathrm{~F} / \mathrm{m}\right)$ and $\mathrm{A}$ is the area of electrode in $\mathrm{m}^{2}$. Combining Equations 3, 4, and 5 yields the electric force generated by the parallel plate capacitor model. Equation 6 adds the additional dependence of the change in distance between the actuator and electrode, $(\Delta \mathrm{d})$, as the actuator deforms toward the electrode.

$$
F_{\text {elec }}=\frac{\varepsilon_{r} \varepsilon_{0} A V^{2}}{(d-\Delta d)^{2}}
$$

The parallel plate capacitor model can be extended to a device that undergoes rotational actuation by including two separate electrodes beneath the actuator. The hinge in this case would act as a torsional hinge resisting the rotation of the device. Figure 7 depicts this type of actuator where the induced electrostatic force acts to rotate the device about the central axis of the hinge.

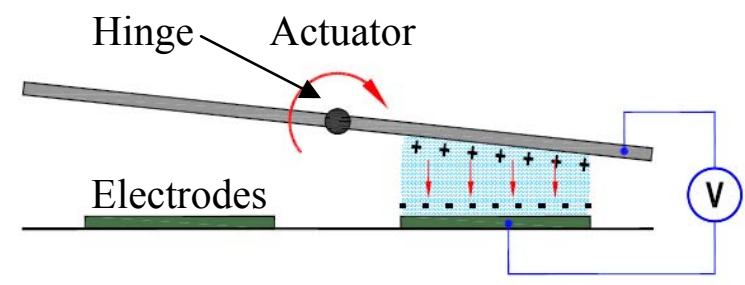

Figure 7. Electrostatic actuation to induce a rotation about a torsional hinge.

From this model it is evident that electrostatic actuation is comprised of a coupling between the electrostatic force and the mechanical restoring force. This electromechanical coupling restricts the ability to examine the electrostatic system 
independently. It is possible, however, to create a test device that can be actuated by applying a direct mechanical force to induce the rotation. The mechanical force can be applied directly to a test device by means of a profilometer stylus. This test method would effectively decouple the electrostatic system from the mechanical system and allow for the direct measurement of the mechanical restoring response during the application of force. The ability to measure the unloading time dependent response is not possible with this method since the actuation force is the weight of the profilometer stylus. If the viscoelastic response is observed in the purely mechanical system, the electrostatic system can be ruled out as a source of the viscoelastic response.

In conclusion, the objective of this research is to design a test device with a torsional hinge that can be mechanically actuated while monitoring the deflection of the test device. The focus of the research is to investigate the effects of applying thin films of gold and aluminum which are commonly used in the fabrication of micro actuators and correlate the properties of these thin films with the viscoelastic response. 


\section{Chapter 3 Testing and Characterization Equipment}

\section{Reliability Test Stand}

To measure the stability and long term reliability of the electrostatic actuator, a reliability test stand (RTS) was designed. The reliability test stand measures the relative angular position of the micro actuators by utilizing a laser beam reflected from the actuator and to a position sensitive detector (PSD). When the laser beam hits the PSD, electron-hole pairs are created and swept away by a voltage placed across the PSD. There are two voltages across the PSD, one running vertically and the other horizontally. These voltages will change depending on the position of the laser beam on the PSD. If the laser moves in the vertical direction, the vertical voltage across the PSD will change. Likewise, if the laser spot moves in the horizontal direction, the horizontal voltage across the PSD will change. In order to monitor the position of an actuator, an initial reading of both the horizontal and vertical voltages from the PSD are measured and recorded. Subsequent voltage measurements can then be recorded to monitor the position of the actuator as a function of time.

The test stand is comprised of three main systems: measurement, position and actuation. These three systems interact with each other and are controlled by custom software using Visual Basic ${ }^{1}$. The software acts on each component prescribing commands and collecting data and acts as a supervisor during testing. This allows for a range of

\footnotetext{
${ }^{1}$ The software is located on the RTS computer at C: Documents and Settings $\backslash$ RTS2-

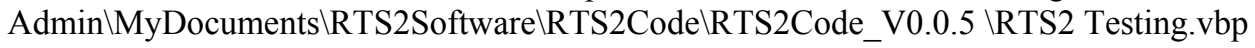


characterization tests that can be performed such as the interaction of electrostatic fields between neighboring actuators, long term (months) actuator stability tests, short term (seconds) actuator response characterization and positional repeatability of the actuators, Figure 8 . The reliability test stand also has the capacity to control temperature to within $\pm 0.35^{\circ} \mathrm{C}$ over a range of temperature between $-10^{\circ} \mathrm{C}$ to $90^{\circ} \mathrm{C}$. Two thermoelectric modules mounted behind the actuators actively control the temperature of the actuator housing allowing for environmental characterization of all the aforementioned testing schemes.

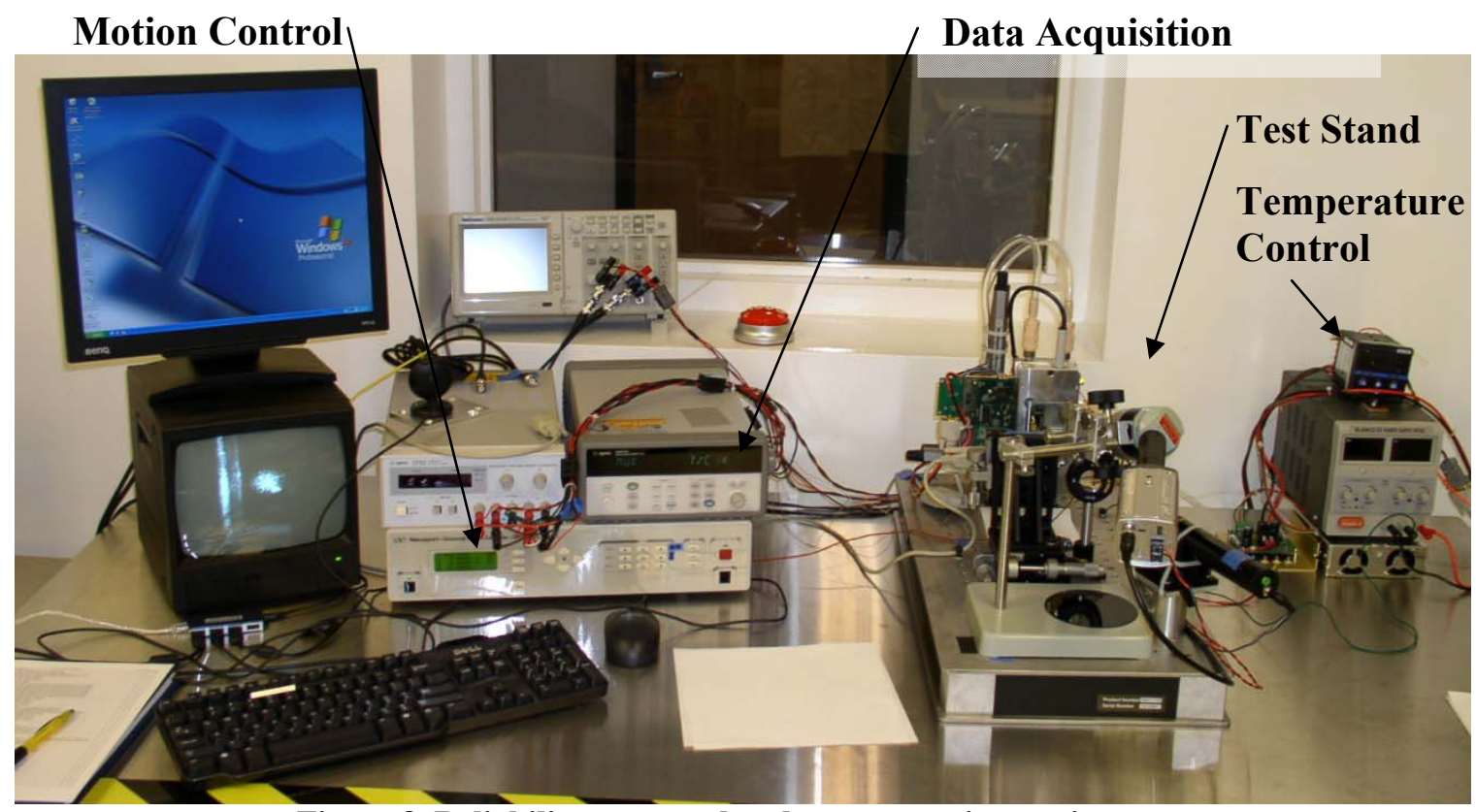

Figure 8. Reliability test stand and accompanying equipment.

The measurement system is comprised of two main components: the data acquisition unit (DAQ) and the position sensitive detector along with its signal amplifier. The DAQ reads the input signals (voltage, resistance and current) from either the sensors attached to the test stand or the voltage output from the PSD amplifier. Each sensor is assigned to 
one of 22 channels available on the DAQ and that particular channel is programmed to measure that signal's input. During testing, data is collected from 16 sensors and recorded to a file on the computer. The main variable of interest when monitoring the rotational position of the actuators is the horizontal voltage $\left(\mathrm{V}_{\mathrm{x}}\right)$ output by the PSD. This voltage is transformed using Equation 7 to find the rotation of the actuator based on the output voltage, $\mathrm{V}_{\mathrm{x}}$, and the perpendicular distance between the face of the actuator and the PSD, $\mathrm{d}_{\mathrm{P}}$.

$$
\operatorname{Rotation}(\text { degrees })=\frac{90}{\pi}\left(\operatorname{AOI}-\left(\operatorname{atan}\left(\frac{1}{\left(\frac{V_{x}}{d_{P}}+\cot (A O I)\right)}\right)\right)\right)
$$

Where $V_{x}$ is the measured voltage from the PSD ranging between -10 to +10 volts, AOI is the angle of incidence and is constant at 1.178 radians $\left(67.5^{\circ}\right), \mathrm{d}_{\mathrm{P}}$ is the perpendicular distance between the actuator and the PSD $\left(\mathrm{d}_{\mathrm{p}}=60 \mathrm{~mm}\right)$. Equation 7 is a geometric transform which requires the angle of incidence (AOI) to be fixed between the incident laser beam and the face of the actuator, Figure 9. 


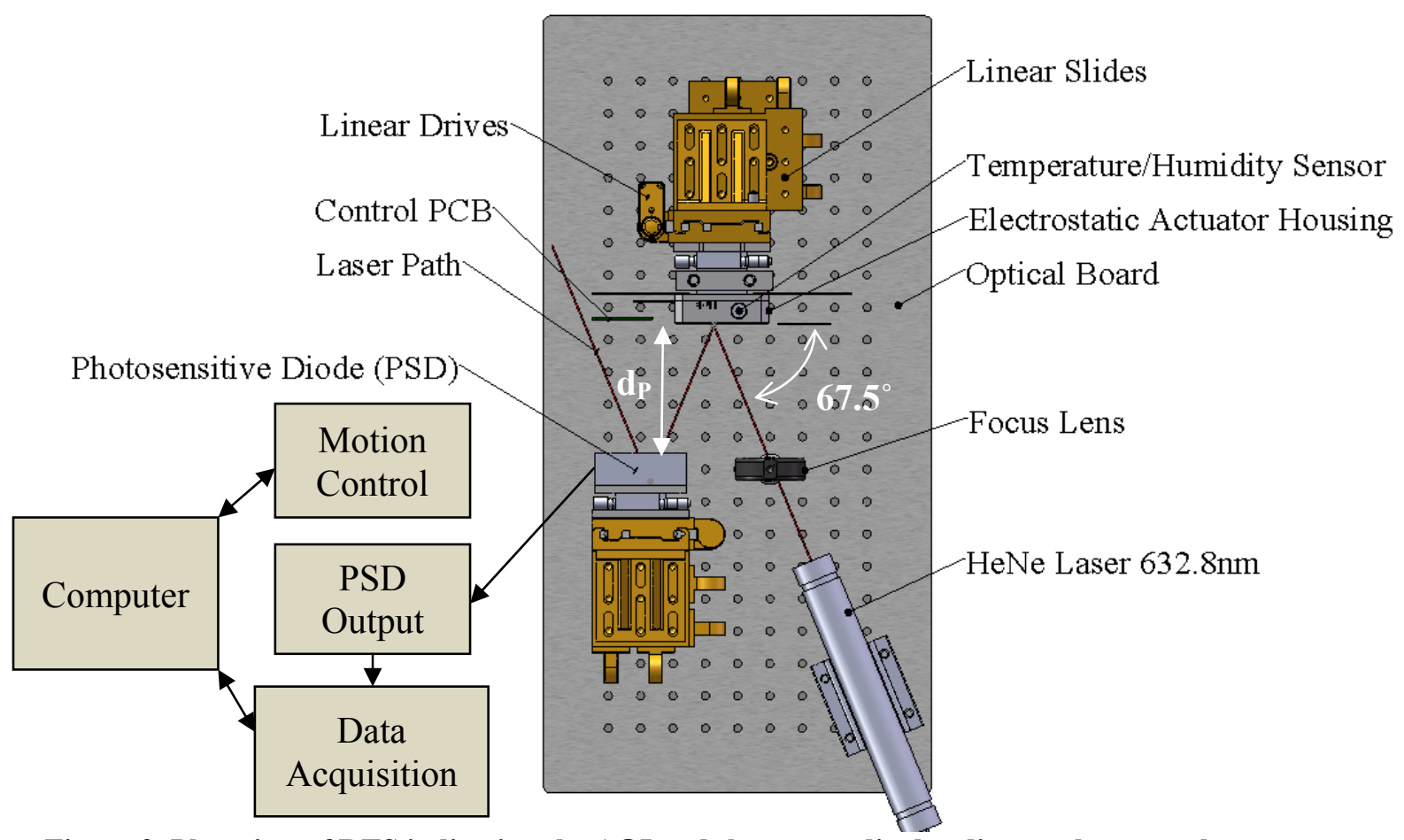

Figure 9. Plan view of RTS indicating the AOI and the perpendicular distance between the actuator and PSD.

In order to verify that the measured viscoelastic response was associated with the electrostatic actuators and not a measurement error due to the RTS, data was collected from two fixed angle mirrors (Figure 10) over a 42 minute time period. The first measurement was collected from a fixed mirror with $0^{\circ}$ degrees of rotation. Immediately following this initial data set, a second set of data was collected from a fixed angle mirror rotated at $-3.88^{\circ}$. 


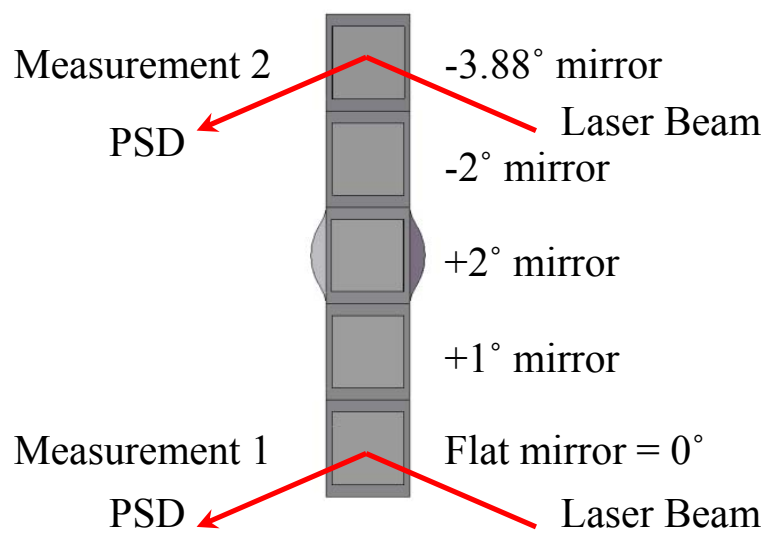

Figure 10. Measurements from fixed angle mirrors verify that the viscoelastic response observed is due to the electrostatic actuators and not a measurement error.

The fixed angle mirror was machined from stainless steel using wire EDM to form the individual faces. $3 \times 3 \times 1 \mathrm{~mm}$ aluminum coated glass mirrors were affixed to the surface of each face using a UV curable adhesive. The main purpose of the fixed angle mirror set is to calibrate the test stand in order to increase the precision of the RTS measurements. A detailed drawing of the fixed angle mirror set is included in Appendix C.

Figure 11 displays the recorded data from both fixed mirrors. The data shows that switching from a $0^{\circ}$ reference mirror to a $-3.88^{\circ}$ rotated mirror does not produce the viscoelastic response. 


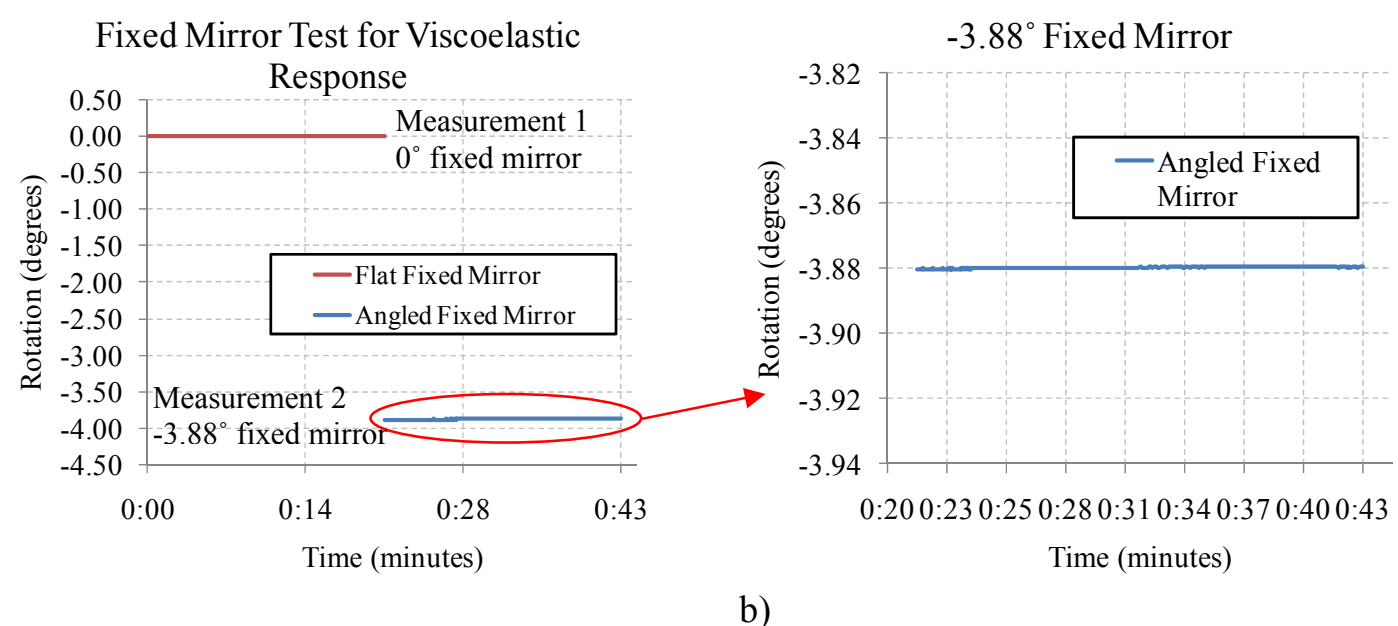

Figure 11. a) Data collected from the fixed angle mirrors verifies that the RTS is not producing the viscoelastic response due to a measurement error b) Detail of $\mathbf{- 3 . 8 8 ^ { \circ }}$ fixed mirror data

Figure $11 \mathrm{~b}$ would show the viscoelastic response if the test stand was producing a measurement error. The metric used to determine the amount of viscoelastic response is the amount of additional deflection that occurs after the initial rotation of the actuator. In effect, this is the range of deflection that is measured during the test duration. The range recorded on the $-3.88^{\circ}$ fixed mirror is $0.001^{\circ}$ indicating that there is no viscoelastic response being measured due to an RTS measurement error and is summarized in Table I.

Table I. RTS Data From Fixed Angle Mirror

\begin{tabular}{|l|r|r|r|}
\hline & $\begin{array}{c}\text { Average Rotation } \\
\text { (degrees) }\end{array}$ & StdDev & Range \\
\hline \hline Flat Mirror & 0.00051 & 0.00042 & 0.00128 \\
\hline Angled Mirror & -3.87972 & 0.00031 & 0.00108 \\
\hline
\end{tabular}

\section{Ambios XP-1 Profilometer}

Profilometry is generally used to measure the surface profile of a material by scanning a cantilevered stylus with a sharp point over the surface and recording the amount of vertical displacement the point undergoes as a function of horizontal distance scanned. Under normal scanning conditions, the object is scanned underneath the stylus head 
where any surface variation is translated into a vertical motion of the stainless steel stylus. A laser is reflected off the head of the stylus and onto a position sensitive detector. Any vertical motion of the stylus results in a beam deflection on a PSD and a subsequent voltage change across a PSD. This voltage is then recorded and transformed into a positional change additionally; vertical movement of the stylus due to surface variation of the material being scanned can be recorded. By disabling the scanning motion of the profilometer stage (static mode), the position of a single point can be monitored for a set time period with a constant force applied. The applied force is simply the weight of the cantilever and stylus head, (Figure 12).

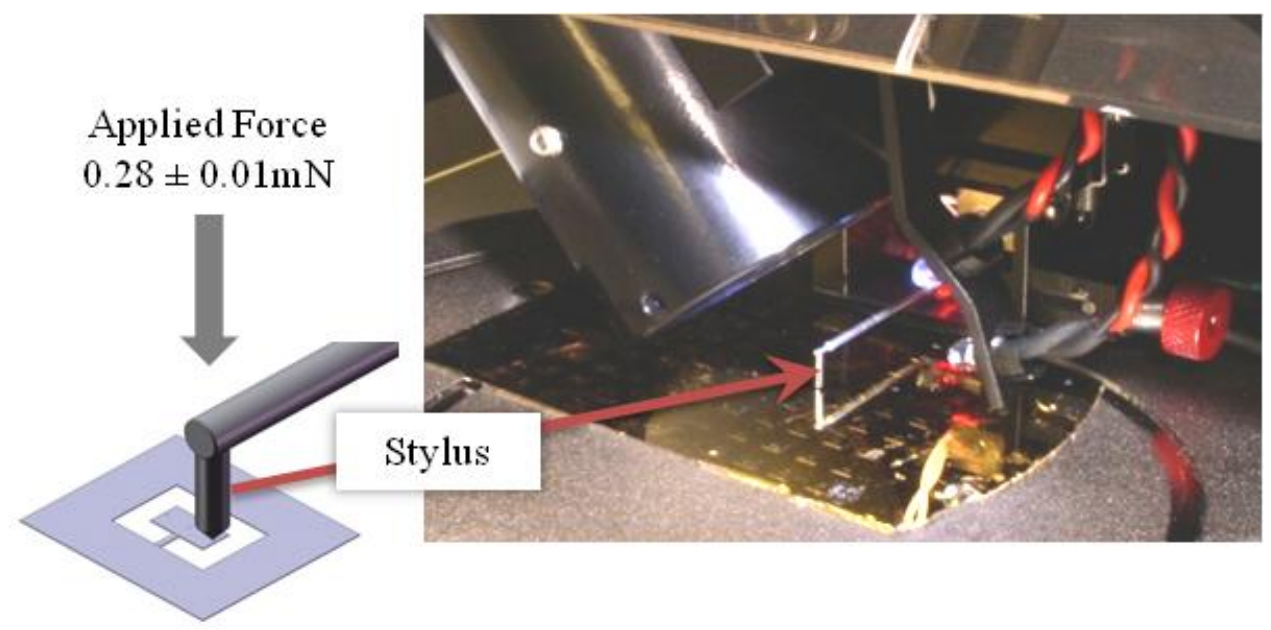

Figure 12. Ambios profilometer viscoelastic measurement process.

The applied force of the stylus was measured using a Gemini-20 milligram scale placed beneath the stylus. The Gemini-20 milligram scale has a load capacity of $20 \mathrm{~g}$ (196.13 $\mathrm{mN})$ and a resolution of $0.001 \mathrm{~g}(0.01 \mathrm{mN})$. Measurements were taken at 10 second intervals for a total time period of 18 minutes to determine the stability of the applied force over time. Figure 13 shows the results of the force measurements from the 
milligram scale where average applied force during the 18 minute period is $0.28 \pm 0.01$ $\mathrm{mN}$.

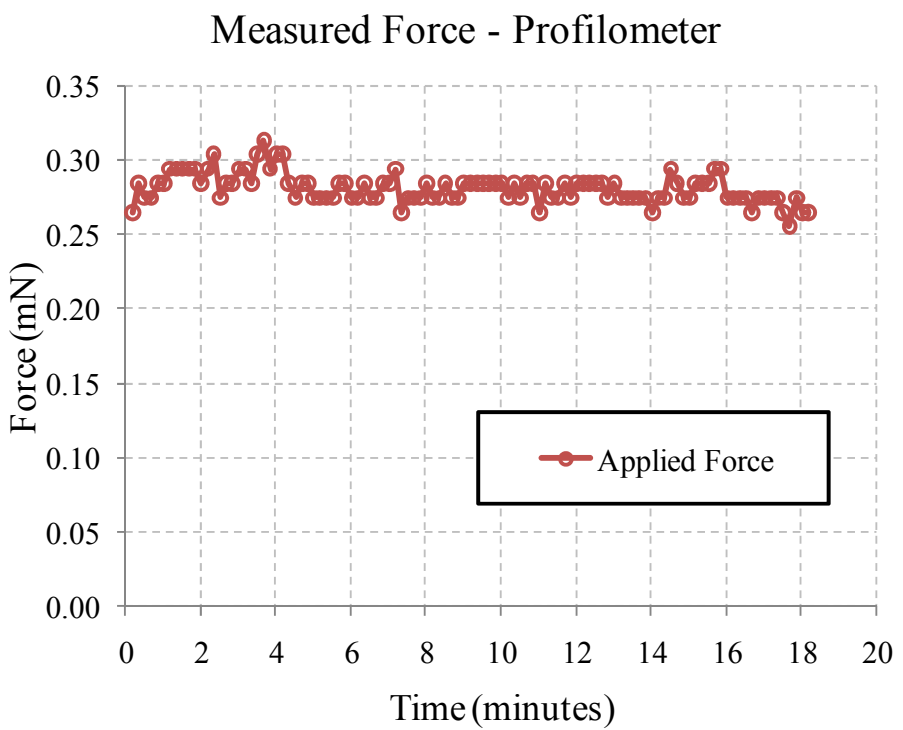

Figure 13. Measured force applied to a milligram scale by the profilometer stylus over an 18 minute time period.

To measure the viscoelastic effect associated with each film type and thickness, the profilometer was set in static mode and each device to be measured was aligned beneath the stylus. The stylus was then engaged onto the device. The force applied during this engagement rotated the device to its initial rotation where the force induced by the stylus was balanced with the mechanical restoring force in the device hinge (Figure 14). The viscoelastic response was then measured over a time interval of 33 seconds at a rate of 10 data points per second. 


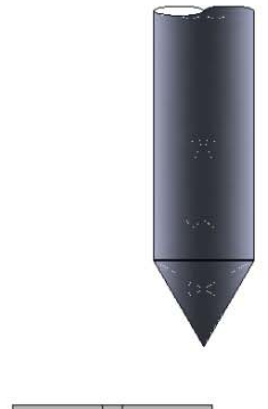

Initial Stylus Alignment Over Device Edge

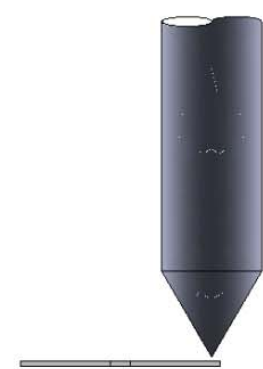

Final Alignment

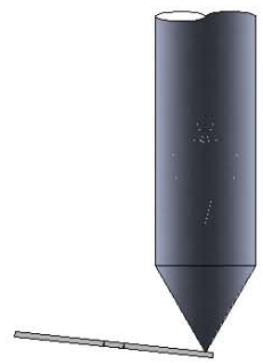

1. Apply Force 2. Start Deflection Measurement

Figure 14. a) Stylus alignment over device b) Engagement of stylus onto edge of device c) Force applied to device to create rotation immediately followed by data logging of deflection.

The background noise level of the profilometer is the limiting factor for the time scale of the tests. Bare silicon devices were torsionally actuated with the profilometer for a time period of 33 seconds to determine the amount of this background noise. Figure 15 shows the average from 15 static scans on 5 different devices indicating that an average drift of $4 \mathrm{~nm}$ occurs over the test period with $20 \mathrm{~nm}$ of background. This $20 \mathrm{~nm}$ of background noise limits the testing time period to 33 seconds by limiting the ability of the profilometer to measure any change in rotation past this 33 second time period. This time period is much less than the reliability test stand time scale $(50 \mathrm{~min})$ due to the amount of background noise and drift of the profilometer system which limits the resolution of this measurement process. 


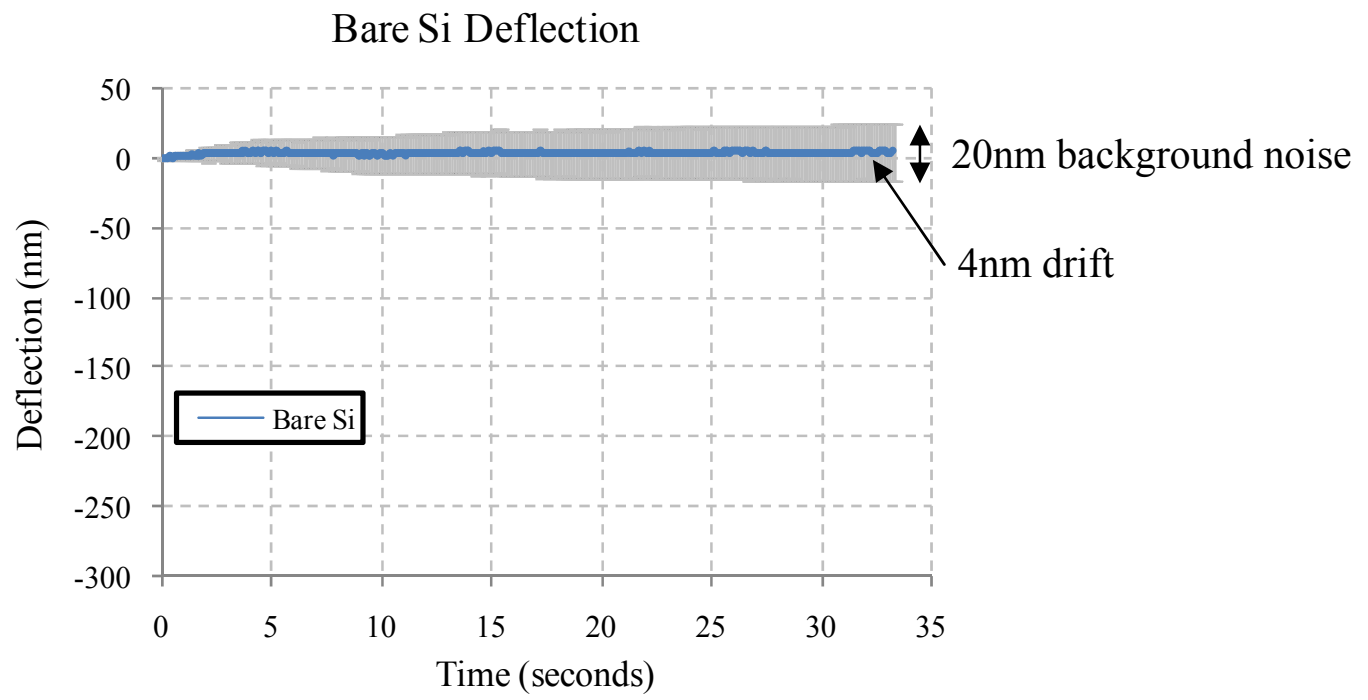

Figure 15. Average reading while monitoring a solid silicon wafer, overall average measurement is $4 \pm 20 \mathrm{~nm}$ of drift \pm the background noise of the system.

Under ideal circumstances there would be no deflection reading during the entire 33 second interval, however, the profilometer is sensitive to physical waves such as the natural vibration of the room and individuals walking near the profilometer as well as music and voices.

Figure 15 also indicates that the bare silicon devices exhibit no viscoelastic response. This is significant for two reasons: (1) it eliminates the silicon substrate as a possible source of the viscoelastic response and, (2) it shows that the profilometer stylus is not sliding on the face of the tilted actuator which could produce a false viscoelastic response. 


\section{Chapter 4 Device Design}

\section{Overview of design and fabrication process}

The objective of the fabrication process was to produce a freestanding fixed-fixed torsional device that, when actuated with $0.28 \mathrm{mN}$ of force applied to the edge, would have an induced stress within the torsional hinge areas comparable to stress levels modeled in the electrostatic actuator hinge design. The viscoelastic response was initially observed in previous research in the electrostatic actuators. By designing the test device to reproduce the same levels of stress modeled in the electrostatic actuator hinge, it is expected that the viscoelastic response will be observed in the test devices if the mechanical element is responsible for the viscoelastic response. Finite element modeling of the electrostatic actuator hinge resulted in von Mises stress levels ranging from 15 $\mathrm{MPa}$ to $26 \mathrm{MPa}$. Figure 16 shows the stress distribution along the electrostatic actuator hinge.
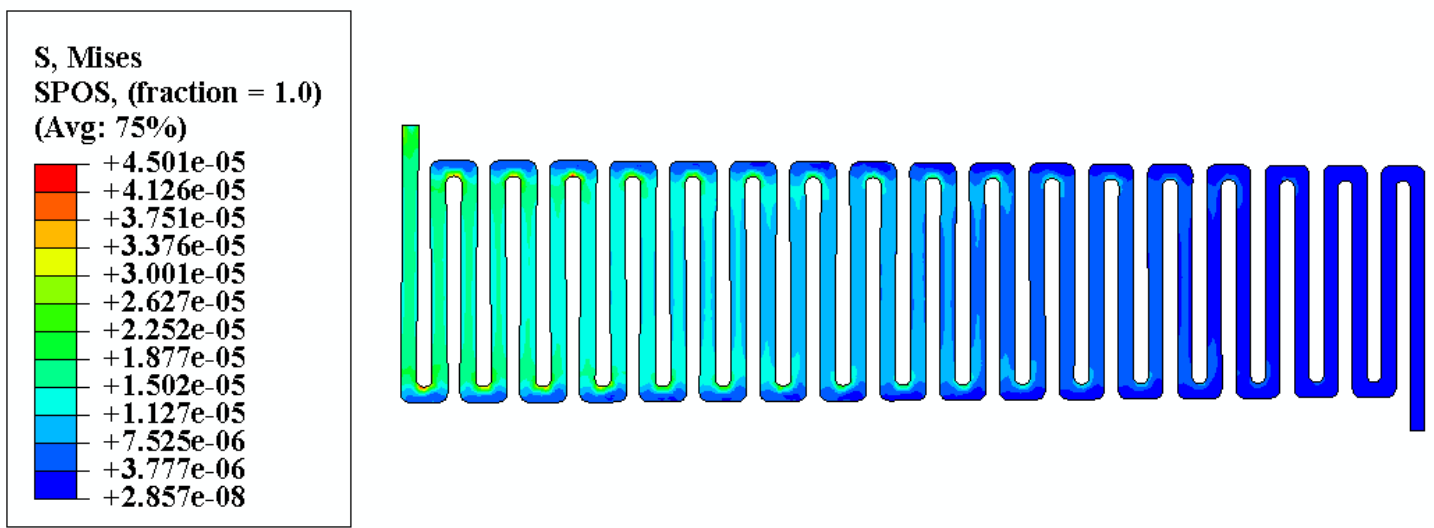

Figure 16. ABAQUS FEM von Mises stress map indicating the stress distribution through the hinge design, $\mathrm{S}$, Mises scale $\times 10^{6}$. 
Based on these modeled stress levels, a simplified torsional hinge design was created so that stress levels would be comparable when a torsional force of $0.28 \mathrm{mN}$ was applied while maintaining fabrication and measurement feasibility, (Figure 17).

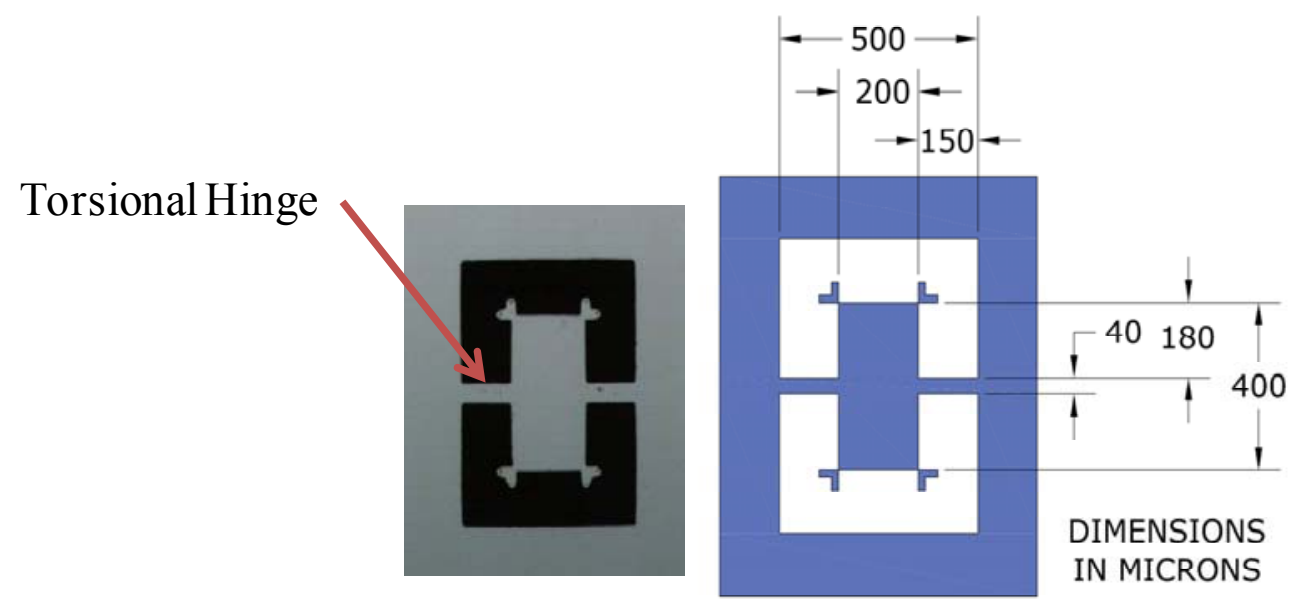

Figure 17. Test device design utilizing $150 \mu \mathrm{m} \times 40 \mu \mathrm{m}$ torsional hinges with an average device thickness of $4.5 \mu \mathrm{m}$.

The stress state across the top of the torsional hinge of the test device ranges from 14 MPa to $20 \mathrm{MPa}$ based on the finite element model (Figure 18 \& 19). The target thickness of the test device was $10 \mu \mathrm{m}$, however, the fabrication of the devices resulted in a final average thickness between the 15 devices of $4.5 \mu \mathrm{m}$. This was due to the inability to control the final through etching process adequately. 

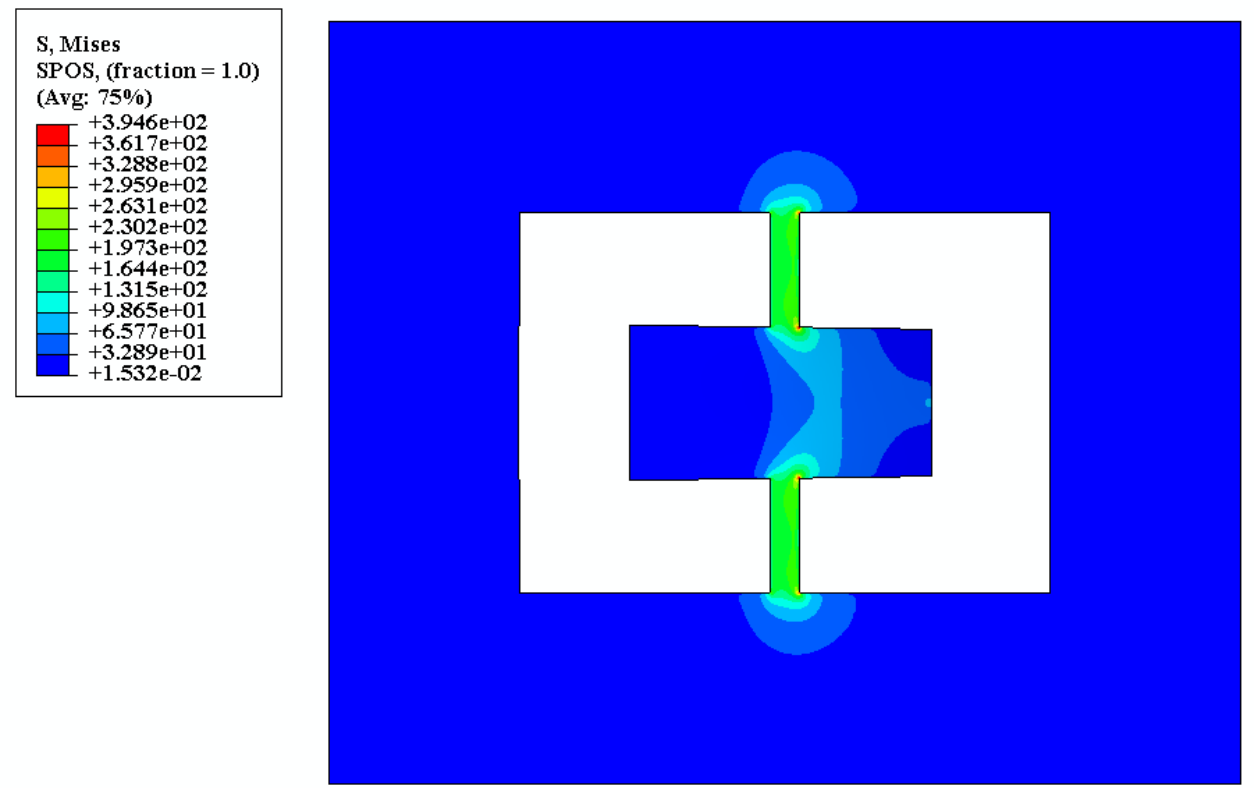

Figure 18. Top view von Mises stress plot of test device indicating 14 MPa to 20 MPa stress distributed across both torsional hinges.
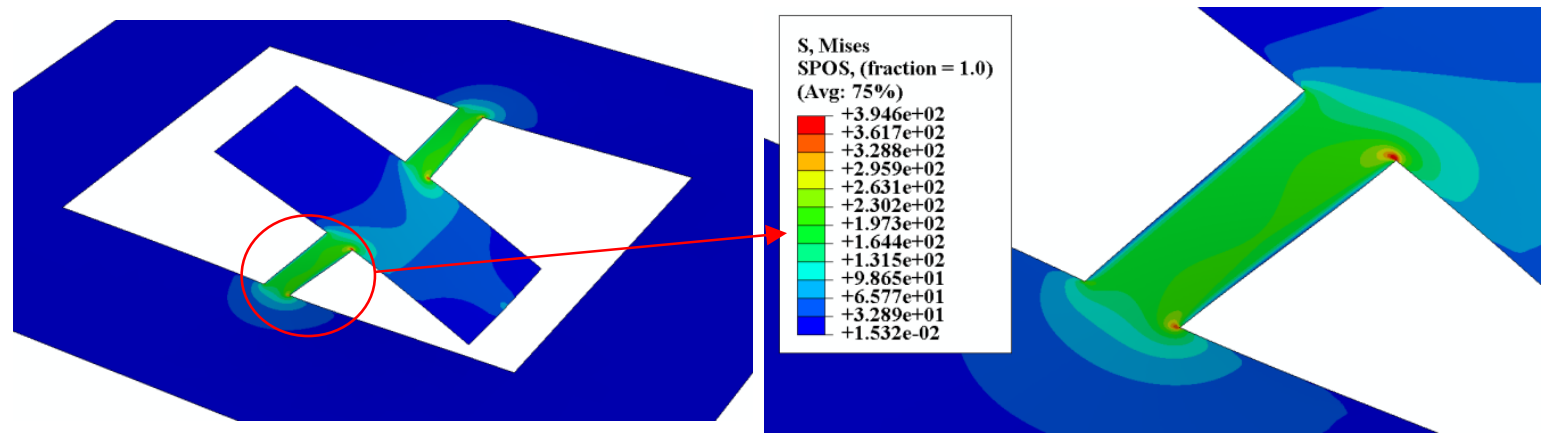

Figure 19. Isometric view of the test device and hinge area indicating the area where the stress is concentrated.

An additional concern in the design of the actuator was the amount of bending that would occur to the $200 \mu \mathrm{m} \times 400 \mu \mathrm{m}$ face of the test device during application of the torsional force. Excessive bending of the device face would distribute the stress induced during torsional actuation throughout the face of the actuator instead of concentrating the stress within the hinge area. 
Figure 20 shows a side and top view of the deformation of the test device with the applied torsional force. The blue area outlines the deformed shape of the test device in Figure 20a indicating minimal bending of the device face. The total amount of deformation is magnified by a factor of 10 .

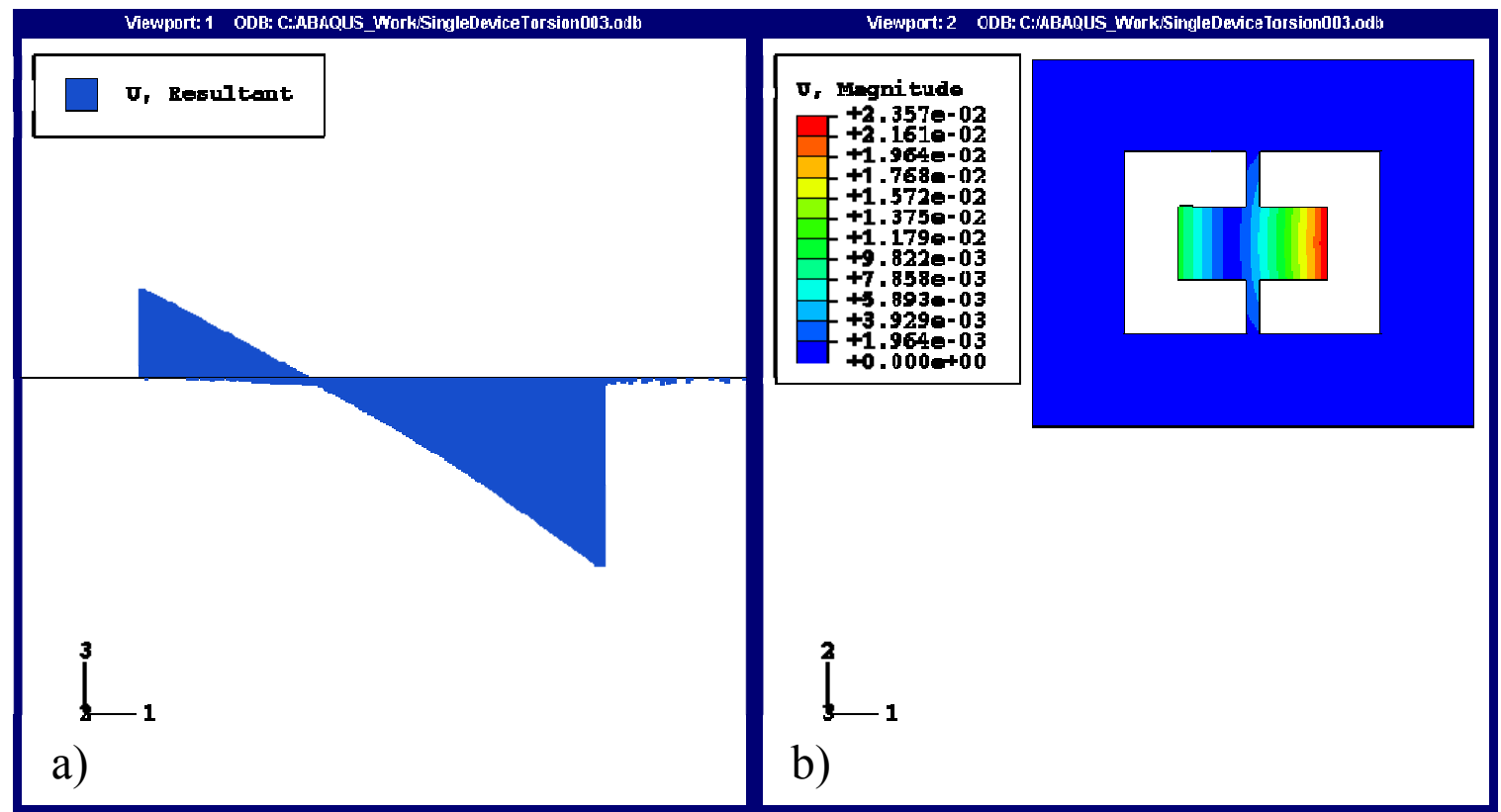

Figure 20. Test device deformation plots a) Deformation symbol plot (10x) showing minimal bending of the actuator face under torsional loading; b) Top view displacement map of test device under torsional load.

Figure 20a also shows that the neutral axis of rotation of the device is not along the axis of the fixed-fixed torsional hinge. This is important when calculating the rotation of the device. Figure 21 and Equation 8 explain how the rotation of the device is calculated from the FEA model. 


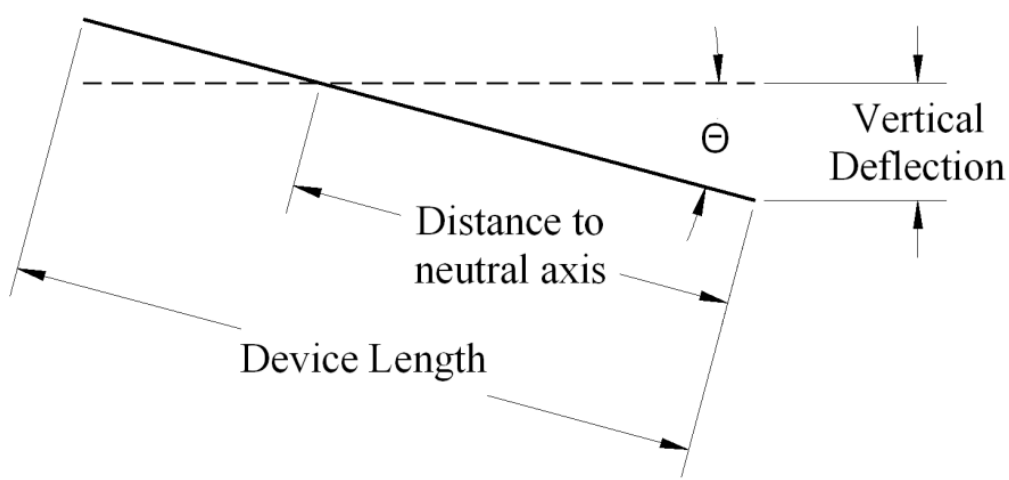

Figure 21. Distance to neutral axis of rotation

$$
\theta=\arcsin \left(\frac{\text { Vertical Deflection }}{\text { Distance to Neutral Axis }}\right)
$$

Figure 22 represents the vertical displacement of individual points along the centerline of the test device model. Using a linear fit to the data and solving for the distance from the edge of the device to the neutral axis of rotation yields a distance of $258.8 \mu \mathrm{m}$. The linear fit equation (Figure 22) also indicates minimal bending of the test device face. The coefficient of determination, $\mathrm{R}^{2}$, value is 0.997 . 
Device Bending Along Center Line of Device Face

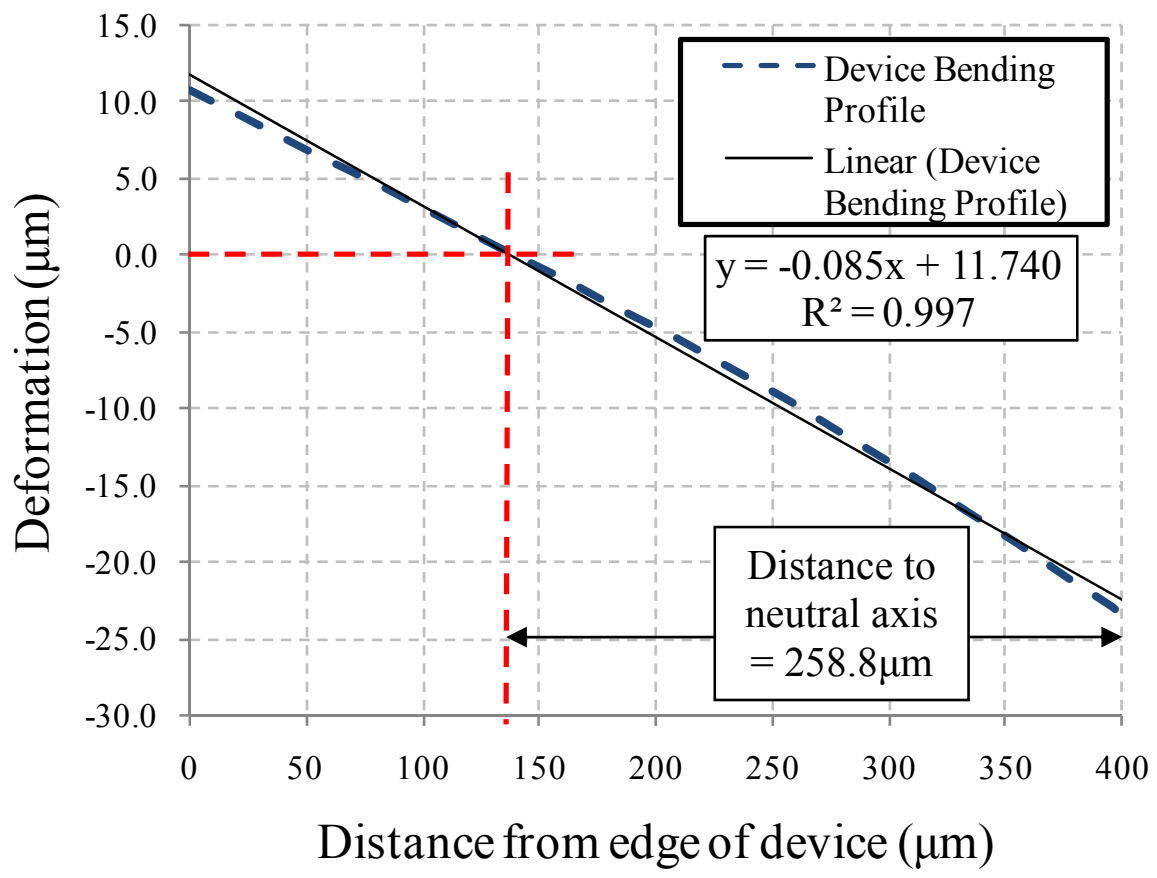

Figure 22. Deformation along centerline of device plate showing minimal bending of device plate under torsional load.

Both the electrostatic actuator model and test device model utilize a shell element (S4R) with reduced integration and 21 integration points through the shell thickness. Additional parameters and mesh convergence for the finite element models can be found in Appendix B. 


\section{Chapter 5 Fabrication of Test Device}

\section{Overview}

The objective of the device fabrication was to create a microscale device using common semiconductor fabrication techniques. A thermal silicon oxide mask was used to protect surrounding silicon areas during the etching process. Bulk micromachining of the silicon was carried out using tetramethylammonium hydroxide doped with isopropyl alcohol at an average rate of $25.90 \pm 0.44 \mu \mathrm{m} / \mathrm{hr}$, (Figure $23 \&$ Table II). The isopropyl alcohol acted to reduce the formation of hillocks on the silicon surface during etching. This process was utilized to create wells on the backside of the $100 \mathrm{~mm}$ (100) oriented silicon wafers. The well depth was controlled so that a thin membrane of silicon measuring 27.4 microns thick remained. Photoresist was then coated on the topside of the wafer and patterned to create the test device outline. Due to pin hole defects in the topside thermal oxide mask, several of the etch wells completely penetrated through the entire thickness of the wafer. These through etched wells acted as alignment markers for the top side device mask with the bottomside etch wells. The thin membranes were then through etched to create the fixed-fixed free standing test devices. The final processing step sputtered the metal layer onto the test structures to form the thin films at two different thicknesses using the parameters listed in Table III.

\section{Fabrication Steps}

1. Wafer cleaning and preparation

a. The wafers were soaked in Piranah $\left(4: 1 \quad \mathrm{H}_{2} \mathrm{SO}_{4}(40 \%): \mathrm{H}_{2} \mathrm{O}_{2}(30 \%)\right)$ for 15 


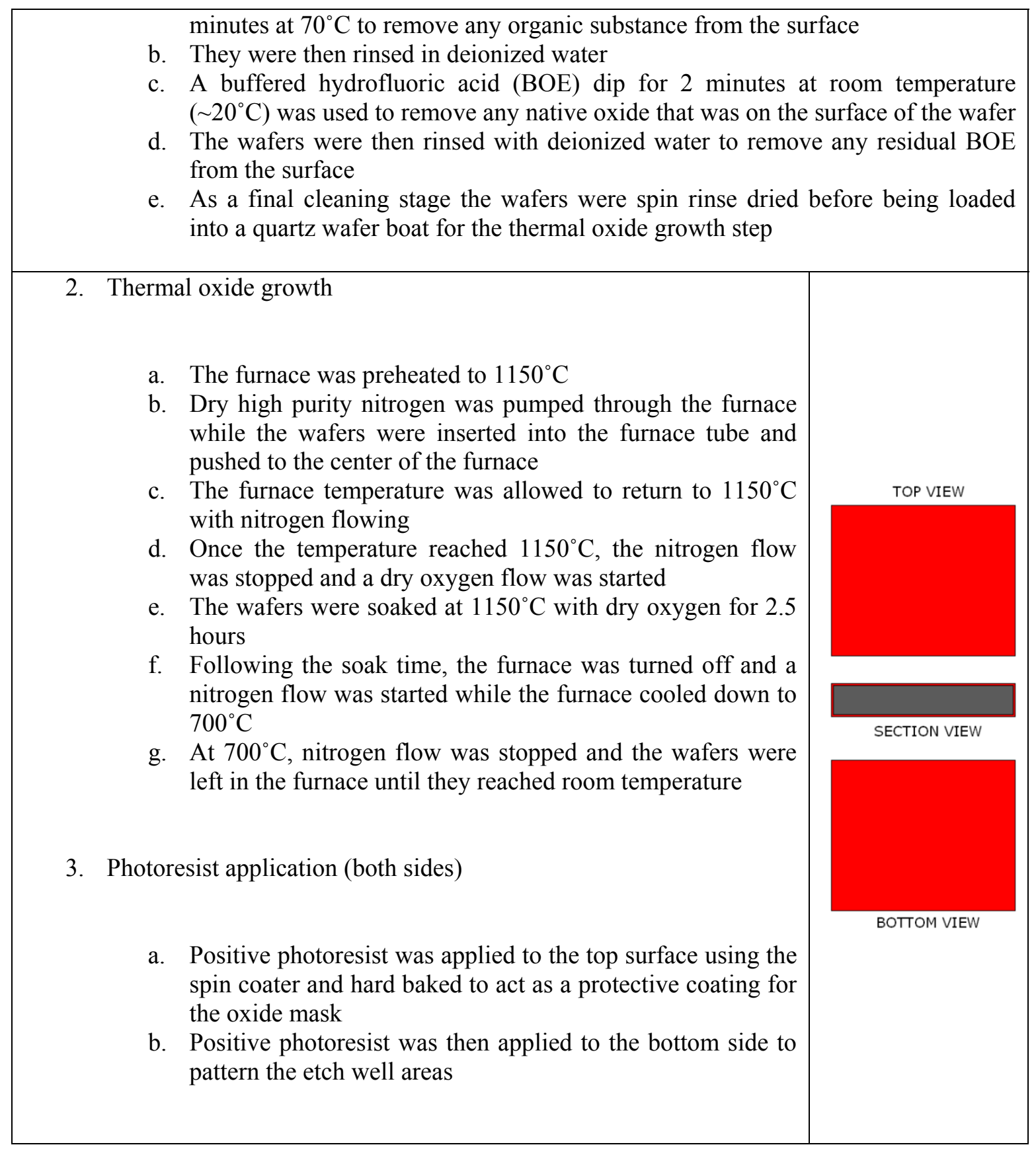




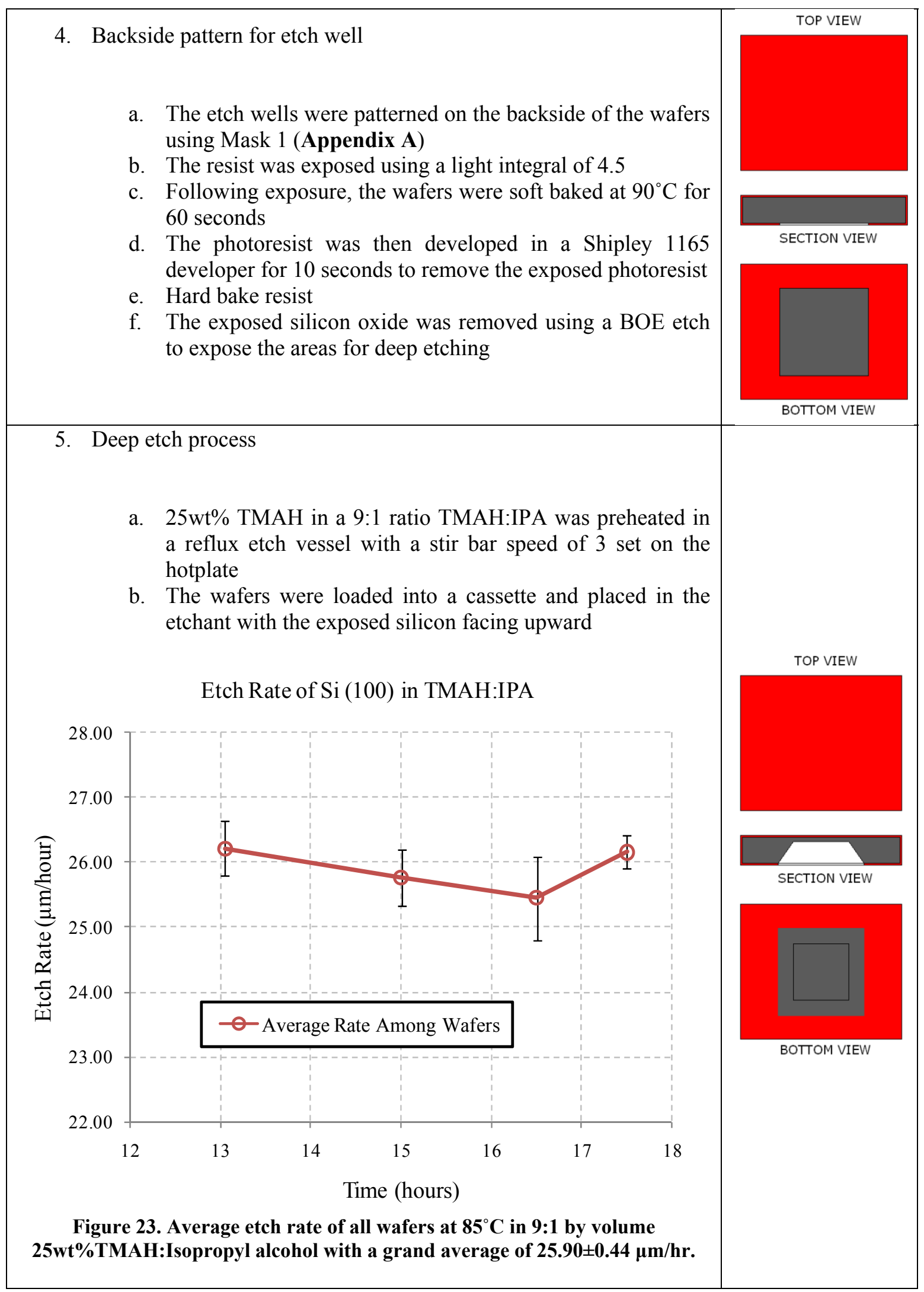




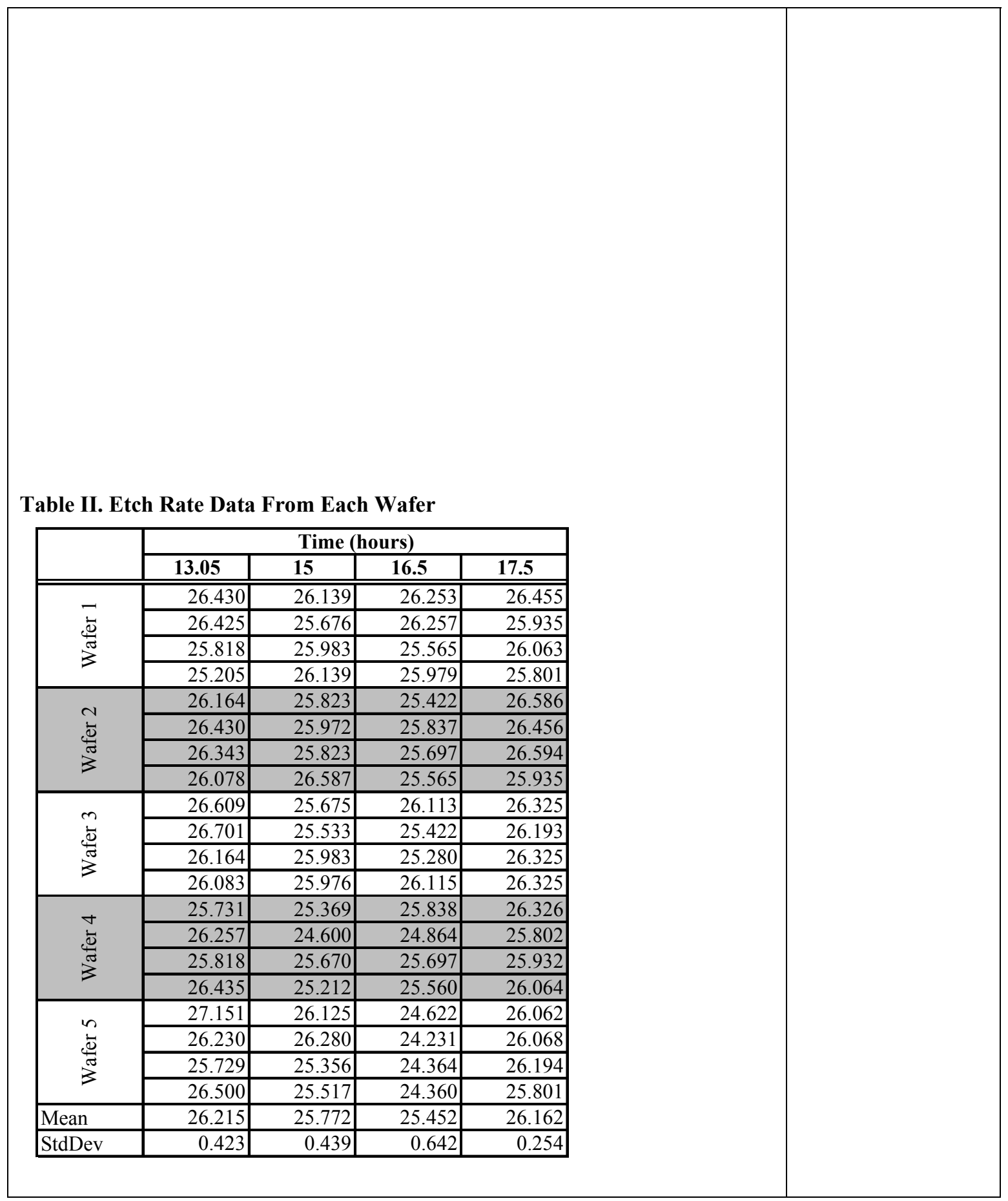




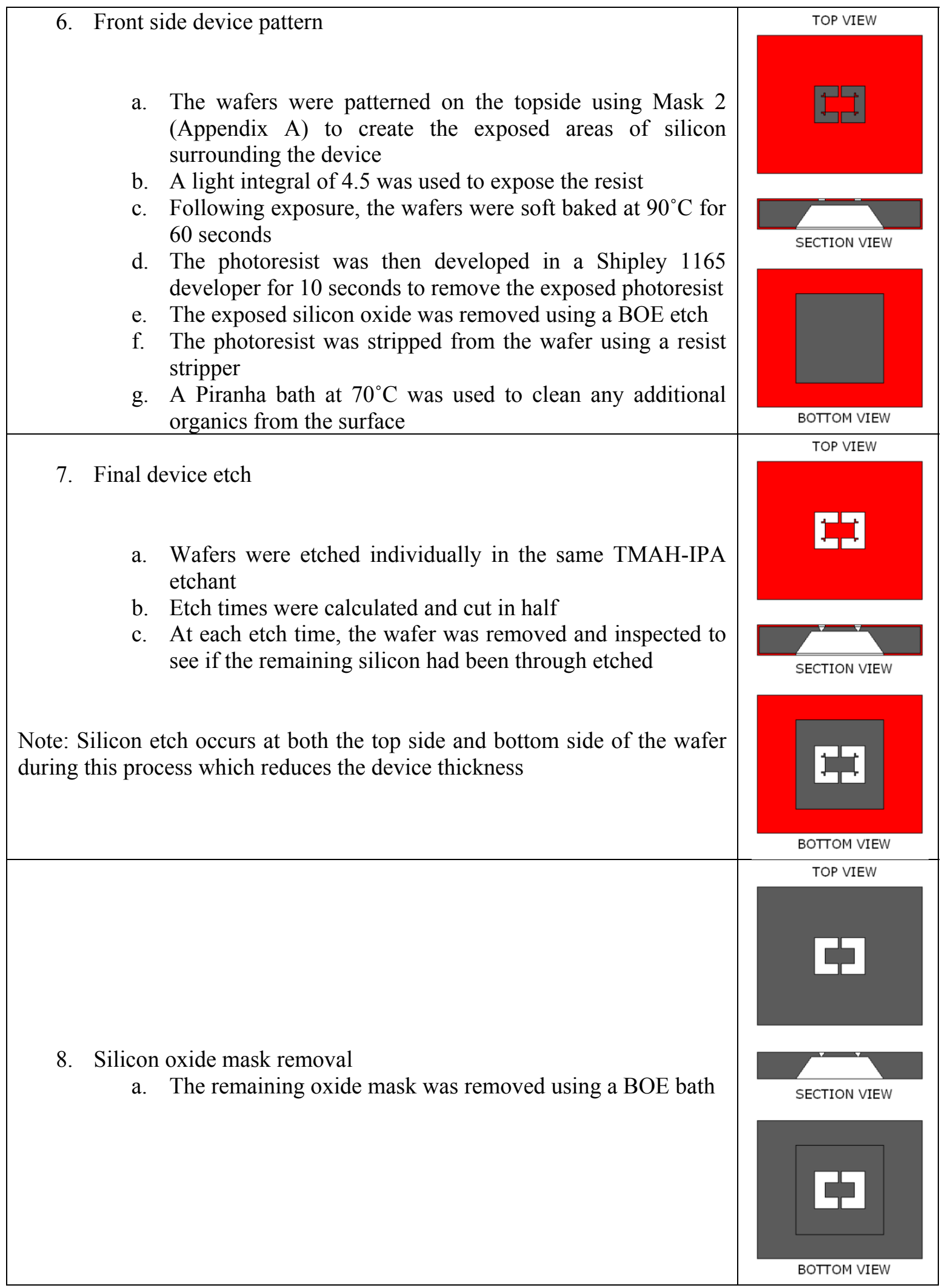




\begin{tabular}{l} 
9. Physical vapor deposition (sputtering) of metal layer \\
Table III. PVD Parameters And Rates Per Film Material \\
\begin{tabular}{|l|r|r|r|r|r|r|r|}
\hline Film Type & Au & Al & Al/Ti \\
\hline \hline Thickness (nm) & 100 & 154 & 100 & 165 & 87.8 & 150 \\
\hline Time (sec) & 214 & 330 & 827.5 & 1620 & 951 & 983 \\
\hline Rate (nm/sec) & 0.467 & 0.467 & 0.121 & 0.102 & 0.092 & 0.153 \\
\hline Power (watts) & 25 & 25 & 30 & 30 & 30 & 30 \\
\hline Pressure (mTorr) & 8 & 8 & 8 & 8 & 8 & 8 \\
\hline \\
\hline
\end{tabular} \\
\hline
\end{tabular}




\section{Chapter 6 Data}

\section{Center Deformation of Test Device}

In order to minimize variation between devices, the same 15 test devices were used for each thin film type and thickness. The bare silicon test devices were initially scanned using the profilometer across both hinges and device face to measure the vertical deformation at the center of the device. Figure 24a shows the path over which the stylus was scanned across the hinge and Figure $24 \mathrm{~b}$ shows a typical scan profile. These scans were used to compare the deflection of the bare silicon devices prior to deposition of the metal film and after each thin film was removed.

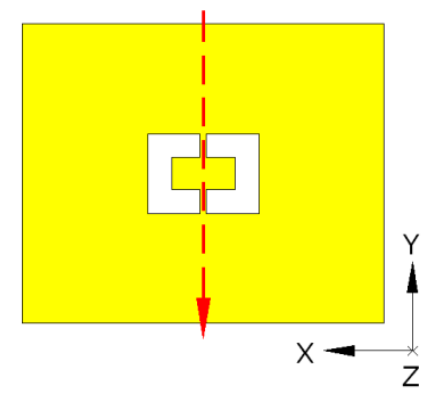

a

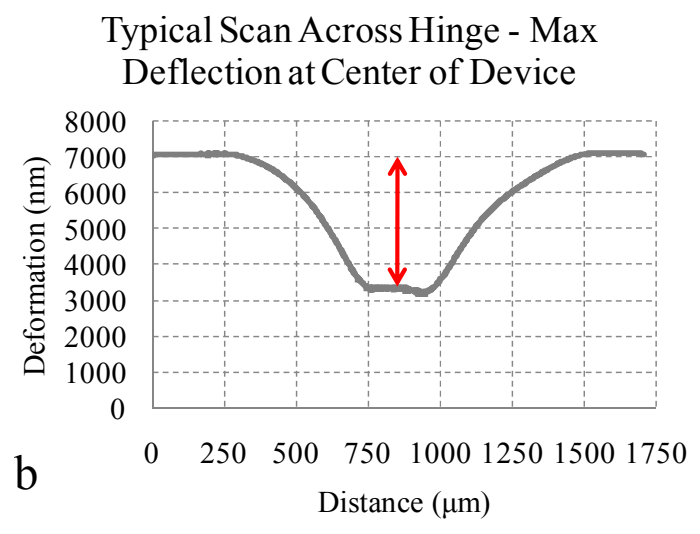

Figure 24 a) The red dashed line shows the scan path across the device hinge b) A typical scan profile showing where the maximum deflection was measured.

Figure 25 illustrates the amount of deflection at the center of each device before metal deposition and after all testing was completed and the metal layer was removed. The results show that the metal films had no impact on the mechanical performance of the silicon substrate; however, the data does show an increasing trend in the amount of vertical deflection of the test devices. 


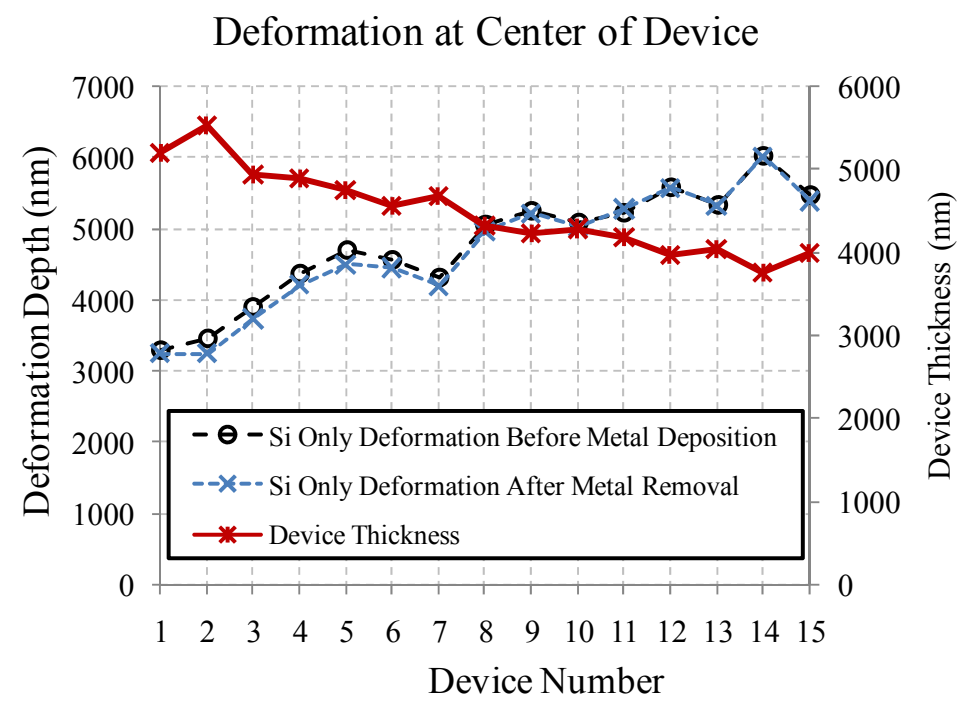

Figure 25. Before metal thin film deposition and after metal layers were tested and removed from the test devices.

The increasing trend seen in the amount of deflection at the center of the device is attributed to the variation in device thickness. The variation of device thickness is attributed to the wet etching process where devices located at the outer edge of the wafer were etched at a higher rate.

\section{Metal Film Thickness}

The thickness of each film was measured by scanning from a bare silicon area to an area coated with the thin film. Table IV lists the average thickness based on 10 scans of each film.

Table IV. Film Thickness Data

\begin{tabular}{|l|r|r|r|r|r|r|}
\hline Film Type & \multicolumn{2}{|c|}{ Au } & \multicolumn{2}{|c|}{ Al } & \multicolumn{2}{|c|}{ Al/Ti } \\
\hline \hline Thickness (nm) & 100 & 154 & 100 & 165 & 87.8 & 150 \\
\hline
\end{tabular}

Prior to measuring the film thickness the profilometer was calibrated using a known step height of $936.6 \mathrm{~nm}$. 


\section{Gold Thin Film}

Viscoelastic behavior was observed in the $100 \mathrm{~nm}$ and $150 \mathrm{~nm}$ thick gold films. Figure 26a shows the average viscoelastic response across the 15 test devices over a period of 33 seconds. A total of $-93.1 \mathrm{~nm}$ of additional vertical movement occurs after the stylus engages the edge of the devices and applies the rotational force. This added deflection corresponds to an additional rotation $(\Delta \theta)$ of 0.021 degrees.

The $150 \mathrm{~nm} \mathrm{Au}$ film also exhibited a viscoelastic response; however, the added thickness of the film increased the total time dependent deflection $(\Delta \theta)$ to $-191.8 \mathrm{~nm}$. Figure $26 \mathrm{~b}$ shows the average viscoelastic response of all 15 test devices over the 33 second scan time. This translates to an additional rotation of 0.042 degrees as a result of the viscoelastic response.
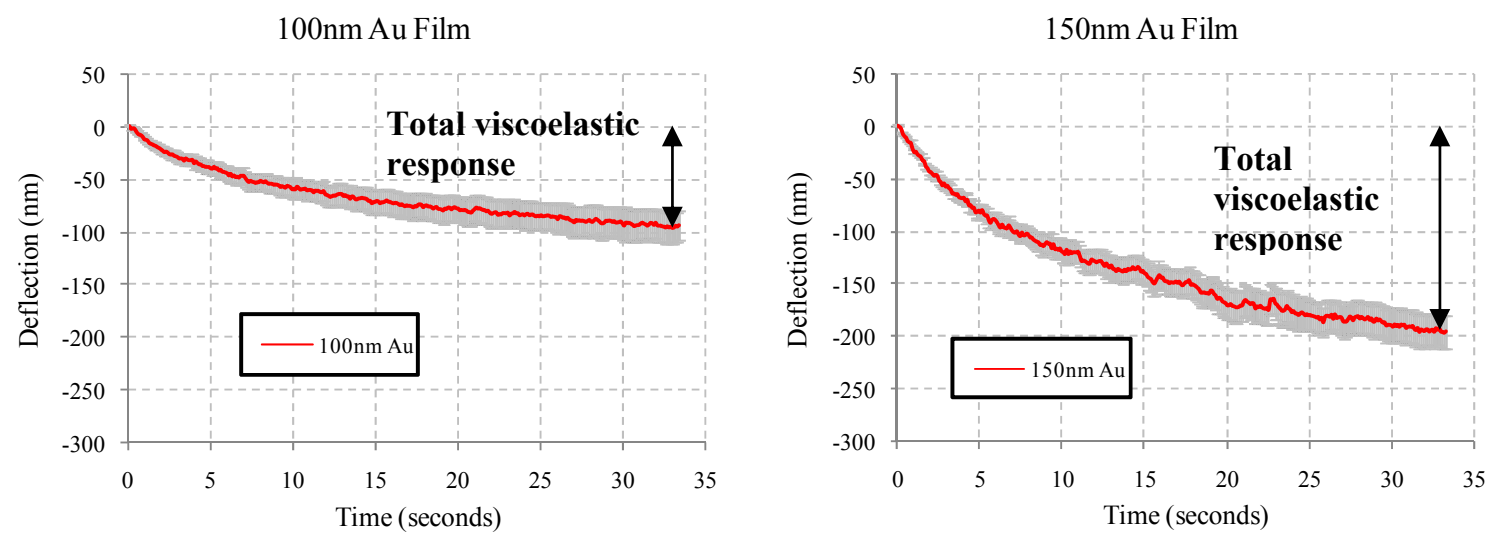

Figure 26. a) Viscoelastic response of $100 \mathrm{~nm}$ gold film b) Viscoelastic response of $150 \mathrm{~nm}$ gold film.

Figure 27 illustrates the increase in the viscoelastic response as a result of the increase in film thickness. Table V summarizes the viscoelastic response where the total deflection 
is measured at the end of the scan. A 106 percent increase between the two thicknesses occurs indicating a viscoelastic mechanism associated with increasing the film thickness.

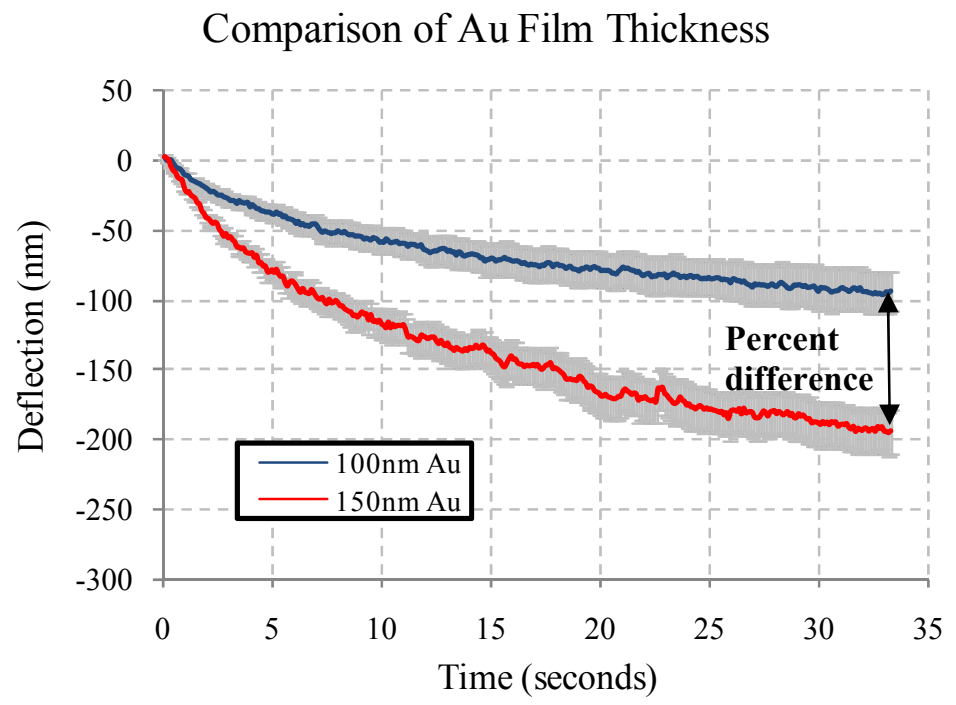

Figure 27. Comparison of viscoelastic response of the gold film.

Table V. Au Thin Film Viscoelastic Response Data

\begin{tabular}{|l|r|r|r|r|}
\hline Film Type & Average Deflection $(\mathrm{nm})$ & StdDev Deflection & Average $\Delta \theta$ (degrees) & StdDev $\Delta \theta$ \\
\hline $100 \mathrm{~nm} \mathrm{Au}$ & -93.1 & 9.47 & -0.021 & 0.002 \\
\hline $150 \mathrm{~nm} \mathrm{Au}$ & -191.8 & 15.52 & -0.042 & 0.003 \\
\hline Percent Increase & 106.0 & & & \\
\hline
\end{tabular}

\section{Grain size analysis}

Backscattered electron images for the $100 \mathrm{~nm}$ and $150 \mathrm{~nm}$ gold films using a solid state backscatter detector are shown in Figure 28. These images indicate that the grain size does not change as a result of increasing the film thickness. The grain size of the two films is estimated to be $100 \mathrm{~nm}$ in diameter. Since the parameters used to deposit the film using PVD were not changed other than the length of time, it is expected that the films would exhibit similar grain size. 

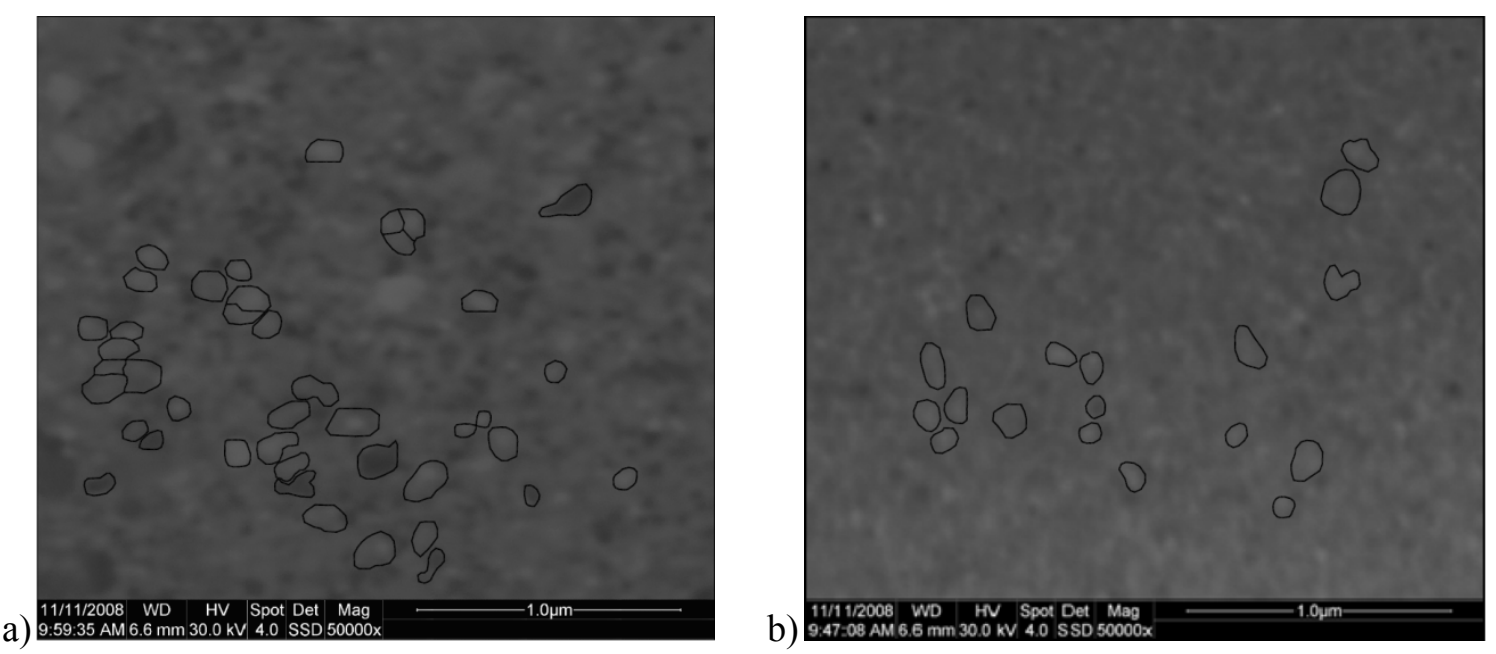

Figure 28. BSE images of the thin gold films indicating grain size is similar between the two films thickness. The dark outlines are added to indicate grain size. a) $100 \mathrm{~nm}$ Au film b) $150 \mathrm{~nm}$ Au film.

\section{Aluminum Thin Films}

Similar viscoelastic results were observed in both the $100 \mathrm{~nm}$ and $150 \mathrm{~nm}$ aluminum thin films, Figure 29. The $100 \mathrm{~nm} \mathrm{Al} \mathrm{film} \mathrm{shows} \mathrm{a} \mathrm{total} \mathrm{of} 76.43 \mathrm{~nm}$ of viscoelastic response. Again, the amount of viscoelastic response increased with the increase in film thickness. The $150 \mathrm{~nm}$ Al film shows a viscoelastic response of $144.14 \mathrm{~nm}$ which is an $88.6 \%$ increase from the 100nm Al film, (Table VI).

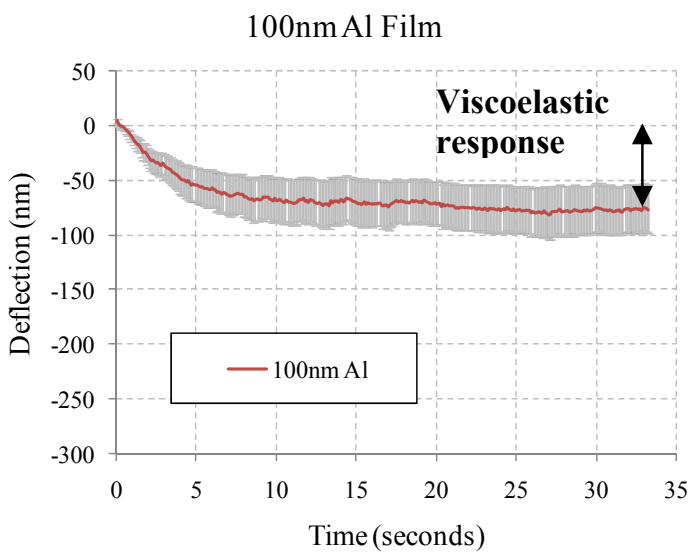

a)

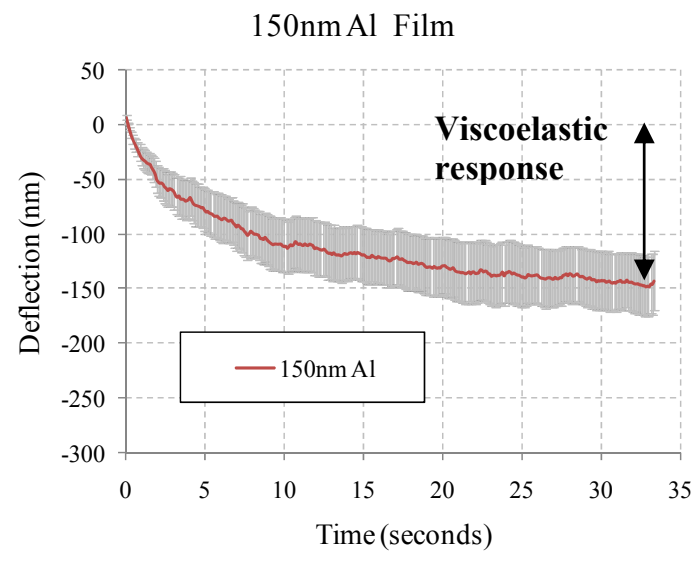

b)

Figure 29. a) Viscoelastic response of the $100 \mathrm{~nm}$ Al film b) Viscoelastic response of the $150 \mathrm{~nm} \mathrm{Al}$ film. 
Figure 30 highlights the increase in the viscoelastic response due to the increase in film thickness.

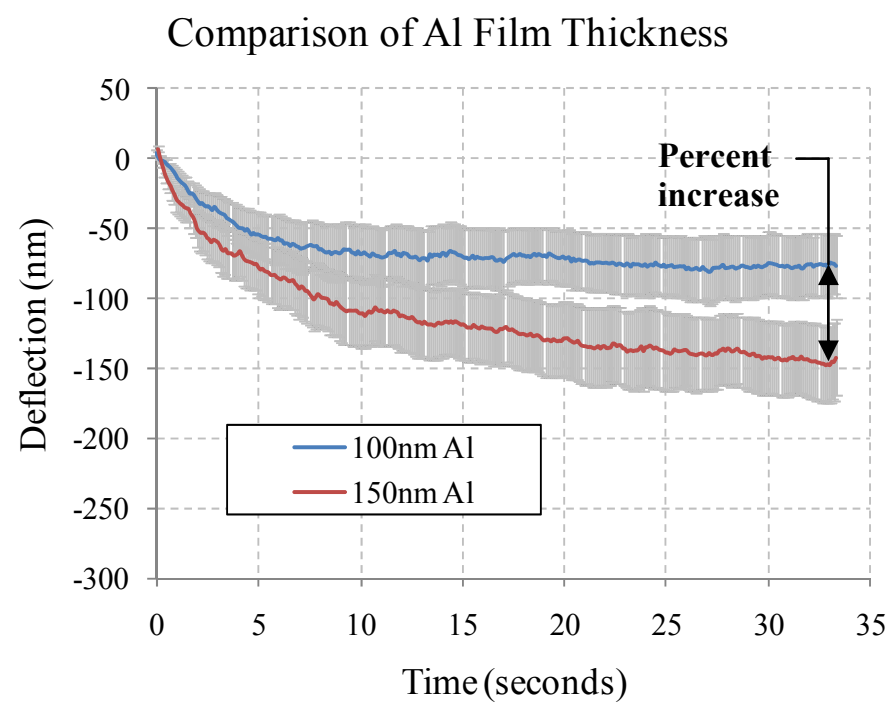

Figure 30. Comparison of the Al film thickness on the amount of viscoelastic response.

\section{Table VI. Viscoelastic Data From Al Thin Films}

\begin{tabular}{|l|r|r|r|r|}
\hline Film Type & Average Deflection (nm) & StdDev Deflection & Average $\Delta \theta$ (degrees) & StdDev $\Delta \theta$ \\
\hline $100 \mathrm{~nm} \mathrm{Al}$ & -76.43 & 22.06 & -0.0169 & 0.0049 \\
\hline $150 \mathrm{~nm} \mathrm{Al}$ & -144.14 & 26.40 & -0.0319 & 0.0058 \\
\hline Percent Increase & 88.6 & & & \\
\hline
\end{tabular}

\section{Grain size analysis}

BSE images reveal a fine grain structure in both film thicknesses. Figure 31a indicates that grains average $100 \mathrm{~nm}$ in diameter for both the $100 \mathrm{~nm}$ and $150 \mathrm{~nm}$ thick aluminum films. The dark outlines were added to the images to indicate the outline of individual grains. 


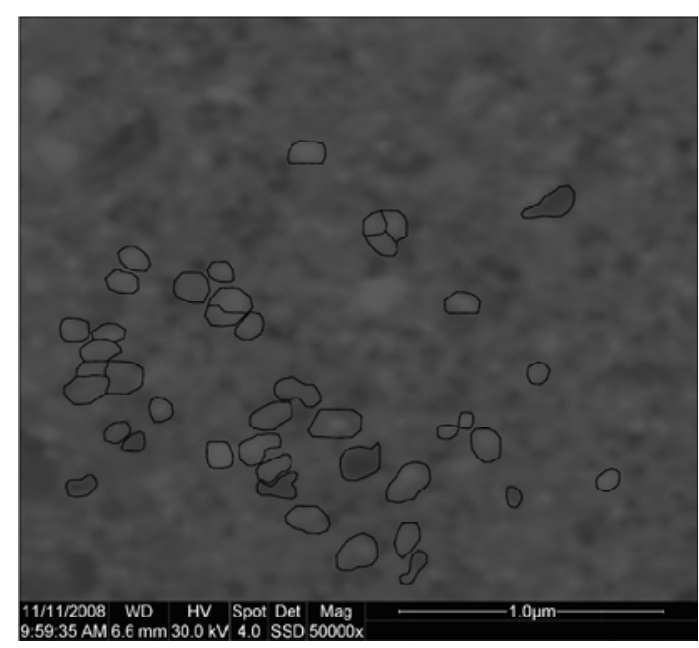

a)

Figure 31. BSE images of the thin aluminum films indicating grain size is similar between the two films thickness. The dark outlines are added to indicate grain size. a) $100 \mathrm{~nm}$ Al film b) $150 \mathrm{~nm} \mathrm{Al}$

films thickness. The dark outlines are added to indicat
film.

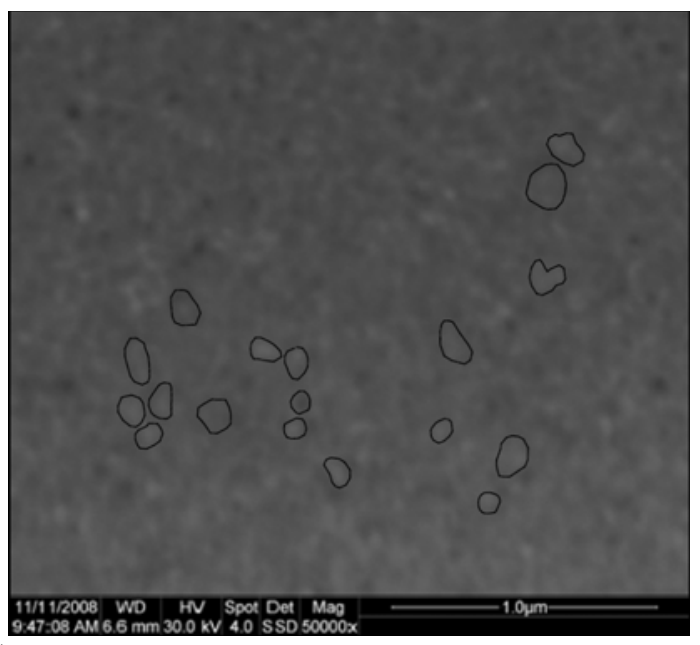

b) 


\section{Chapter 7 Discussion}

\section{Viscoelastic Mechanism - Theory}

\section{Point Defect Diffusion at Grain Boundaries}

In order to reduce or eliminate this viscoelastic effect it is necessary to understand the mechanisms responsible for creating the time dependent response. The following are two theoretical models that have been introduced to explain the fundamental mechanism responsible for this effect. Both models deal with a time dependent process where defects within the thin film crystal structure are responsible for the effect.

The first, introduced by Zener in 1948, describes a mechanism where point defects along grain boundaries diffuse along the grain boundary (8). These point defects pile up at points of intersecting grains called triple points. The point defects are then inhibited from further diffusion along the boundary by the triple points. This allows for mass transport along the grain boundary which is time dependent and will eventually reach an equilibrium point where defect diffusion will no longer take place and the material will no longer deform. When the force is removed from the material, the pile up of point defects will diffuse back along the grain boundary. This re-distribution of point defects allows for the material to fully recover the viscous deformation. Figure 32a depicts the triple point intersection of three grains, the point defects which create the grain boundary surfaces and the dislocation defects within the grain boundary when no stress is applied. The model assumes that the boundaries are pinned only at the triple points which allows 
for the defects at the surface of the grain boundaries to diffuse toward the triple points under an applied stress, Figure 32b. With the assumption that the grains are only pinned at the triple points, it is expected that as grain size decreases, the length that is available for defects to diffuse along will also decrease, thereby decreasing the viscoelastic response.

a)

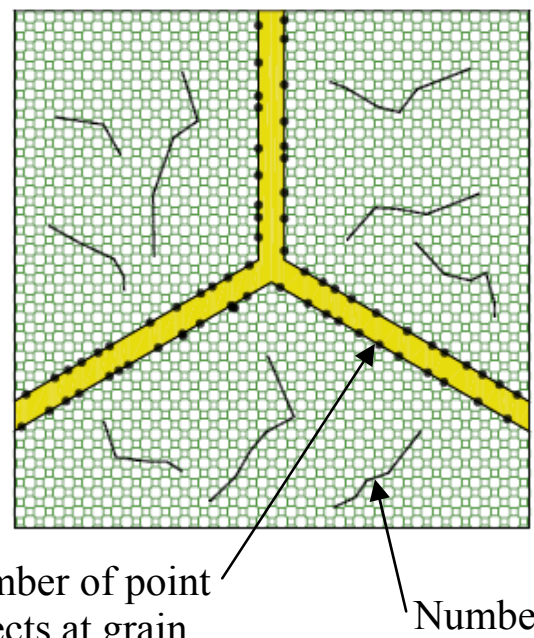

defects at grain

boundary $=\mathrm{N}_{\mathrm{b}}$
Point defects diffuse to triple points

$$
\text { within grains }=\mathrm{N}_{\mathrm{g}}
$$

b)
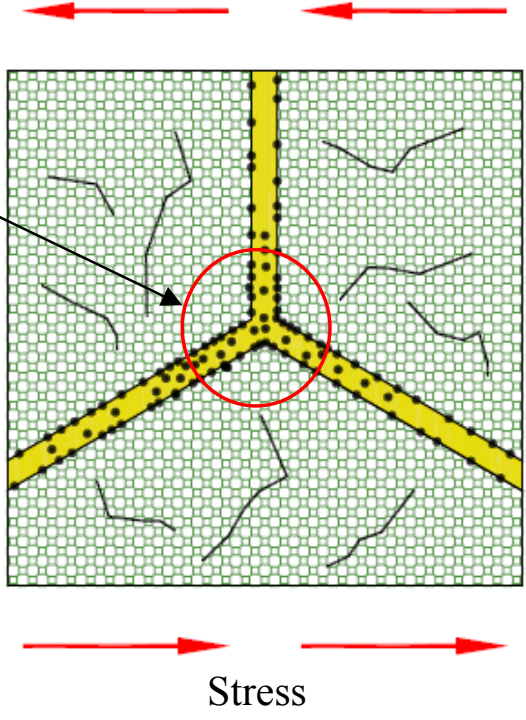

Figure 32. Point defects along grain boundaries diffuse to grain triple points under applied stress.

Conversely, for a large grain diameter, the viscoelastic response would increase indicating that materials with large grains such as bulk materials would exhibit large amounts of viscoelastic response. This is not the case with bulk materials. This incongruity can be resolved when looking at the ratio of the number of defects at the grain boundary to the number of defects within the crystal lattice. Looking at the surface area to volume ratio of various grain sizes, the ratio increases as grain diameter decreases, Figure 33. 


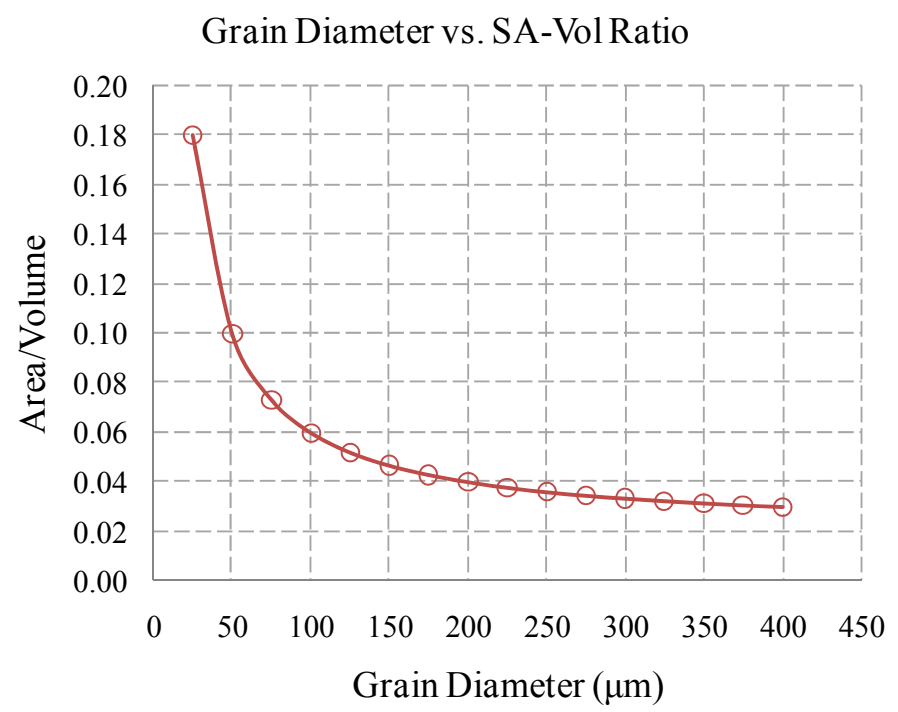

Figure 33. Surface area to volume ratio for various grain diameters.

As this ratio increases, the ratio between the number of defects at the surface of the grain to the number of defects within the grain will also increase. The ratio between the number of defects at the grain boundary $\left(\mathrm{N}_{\mathrm{b}}\right)$ to the number of defects within the grain $\left(\mathrm{N}_{\mathrm{g}}\right)$ will follow the same trend as the grain size decreases. This indicates that there will be a greater viscoelastic effect as grain size decreases due to the number of defects available at the grain boundary surface versus the number of defects within the grain itself. Additionally, a larger grain will have a greater number of facets, or pinning points, available to limit the viscoelastic effect in bulk scale materials. Overall, the Zener model suggests that the viscoelastic effect scales in relation to the ratio of defects at the boundary and within the grain. This resolves the observation of the viscoelastic effect measured within the thin films but does not resolve why the effect increases with film thickness. Backscattered electron (BSE) images of the films show that grain size between the film types and thicknesses does not change. Since the viscoelastic effect scales with the ratio between defects, $\mathrm{N}_{\mathrm{b}} / \mathrm{N}_{\mathrm{g}}$, if the grain diameters are similar between 
film thicknesses, it is expected that the viscoelastic effect would remain constant and independent of film thickness. This is not shown to be the case, however; data from both film types shows an increase in viscoelastic behavior as the film thickness increases. This signifies that there must be an additional mechanism beyond viscous flow of grain boundary defects. This additional mechanism can be accounted for by considering the defects within the grains.

\section{Dislocation Bowing}

The second theory considers the motion of dislocations within the crystal structure of the metal grains. Nowick and Berry introduced the model of dislocation bowing within the crystal lattice under an applied shear stress (9). The theory describes that a dislocation that is pinned at two points within the lattice will be able to bow under an applied shear stress by atomic diffusion of vacancies and atoms along the dislocation line. In order for the dislocation line to bow outward, atoms jump from adjacent lattice sites into the dislocation core. Tension along the dislocation line increases as the dislocation bows outward as a result of this diffusion process. The driving mechanism for this diffusion process is the applied shear stress that results from the material deforming under an applied force. When the shear stress is removed, the line tension of the dislocation bow provides the mechanism for deformation recovery as the line tension causes the dislocation to straighten to its original configuration. Figure 34 depicts how the dislocation can bow outward under an applied shear stress. 


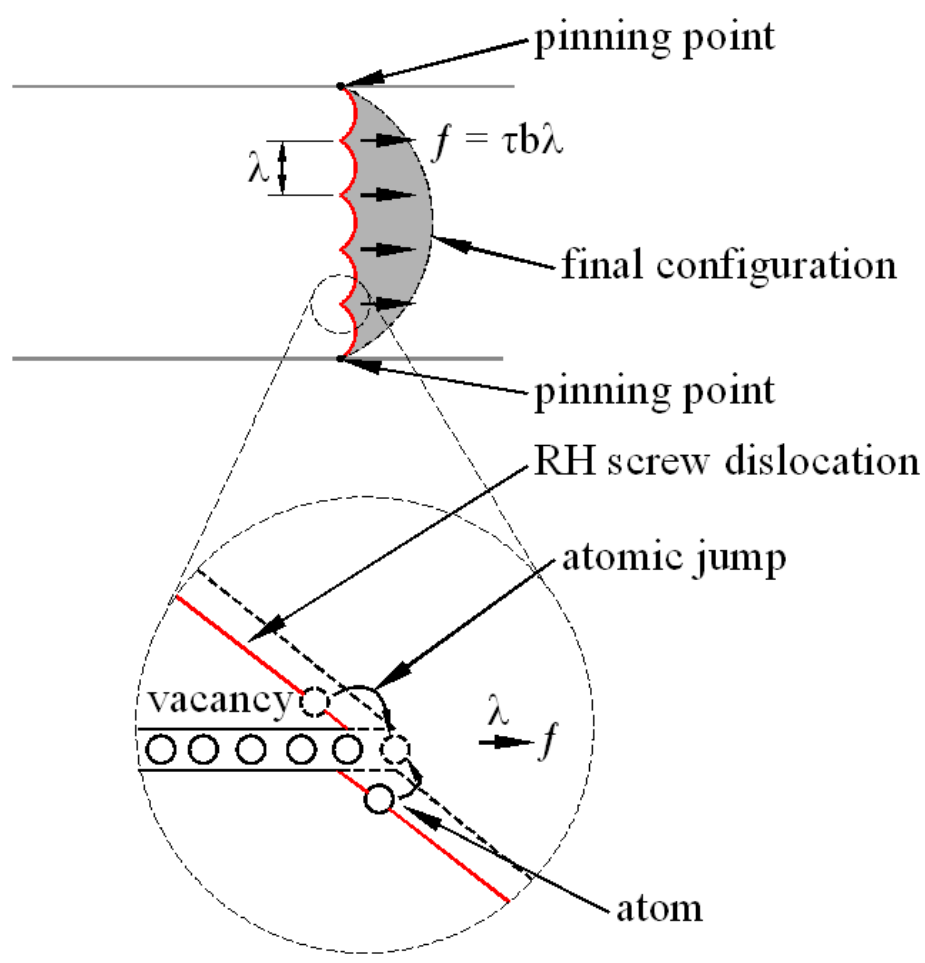

Figure 34. Dislocations within grains bow under an applied stress through pipe diffusion along the dislocation core where atomic jumps leave vacancies behind (10).

The red line in Figure 34 depicts a dislocation extending between two pinning points. A force $(f)$ will develop along the length of the dislocation at positions separated by a distance $(\lambda)$. The force is a function of the shear stress, Burgers vector of the dislocation and the distance between the points at which the forces are acting along the dislocation core. The dislocation will bow in the direction of the force by the process of atomic diffusion at the dislocation core. As the dislocation advances, atoms jump into the dislocation core and leave vacancies behind. This allows the process to continue until the dislocation has reached an equilibrium point where the line tension of the dislocation is equal to the driving force of the shear stress. Upon release of the shear stress, the dislocation line tension developed during bowing pulls the dislocation back to its original configuration in a reversal of the process. This second theory of dislocation bowing 
allows for an increase in viscoelastic response due to film thickness. Since the driving force is only dependent on the shear stress, Burgers vector of the dislocation and the distance between the points at which the forces are acting along the dislocation core and not dependent on grain size, for a given amount of shear stress the viscoelastic response will increase with the number of grains. Since the grain diameter between the films is similar, adding thickness to the film increases the number of grains. As a consequence, the number of dislocations that are available to undergo bowing is increased resulting in an increase in the amount of deformation.

Ultimately, the viscoelastic response observed in the test devices and the viscoelastic response observed in the electrostatic actuators is a combination of these two mechanisms. In attempting to control the response there are several avenues that are available. As mentioned earlier, the device position of the electrostatic actuators can be controlled through the manipulation of the electrostatic force acting on them. This method has several drawbacks, however. The viscoelastic effect would need to be characterized for each actuation scheme. This type of characterization is time intensive and if any of the deposition processes which influence grain size and/or film thickness change during fabrication, the characterization would need to be repeated. Once the viscoelastic response was fully characterized, a program would need to be created that could adjust the electrostatic force controlling the position of the actuator continuously to counteract the viscoelastic response. While this type of characterization and control are possible, an alternative method to reduce or eliminate the viscoelastic response is possible. Both mechanisms responsible for the viscoelastic response depend on the 
motion of defects within the material. If the defect motion was limited, then the viscoelastic response would be reduced or possibly eliminated. A common method used to inhibit dislocation motion is through the formation of a second phase precipitate that acts to block defect motion. By sputtering a thin film alloy that has a solute concentration greater than the limits of solubility and then heat treating the film, a fine dispersion of particles will be created within the crystal lattice and at the grain boundaries. These fine particles will act to block defect motion along the grain boundary and limit the amount of dislocation bowing that can occur. Additionally, a portion of the alloying element will remain in solid solution. These solid solution atoms will act to distort the crystal lattice, thereby creating localized stress fields. These stress fields will also act to inhibit defect motion and dislocation bowing. 


\section{Al-Ti Thin Films}

AlTi thin films were sputter deposited onto the test devices to investigate if the viscoelastic response could be reduced or eliminated through the addition of an alloying component. A sputtering target consisting of 0.30 at $\% \mathrm{Ti}$ was used to create the thin films. In a study investigating dilute titanium in aluminum thin films, it was shown that Ti will remain in solid solution up to 0.20 at $\%$ (11). Following the sputtering process, the test devices were heat treated at $500 \mathrm{~K}$ for 45 minutes to form an $\mathrm{Al}_{3}$ Ti precipitate (11).

Figure 35 shows the test results for the $100 \mathrm{~nm} \mathrm{Al} \mathrm{thin} \mathrm{film} \mathrm{and} \mathrm{the} 100 \mathrm{~nm} \mathrm{AlTi}$ thin film. The addition of Ti effectively eliminated the viscoelastic response observed in the $100 \mathrm{~nm}$ aluminum thin films.

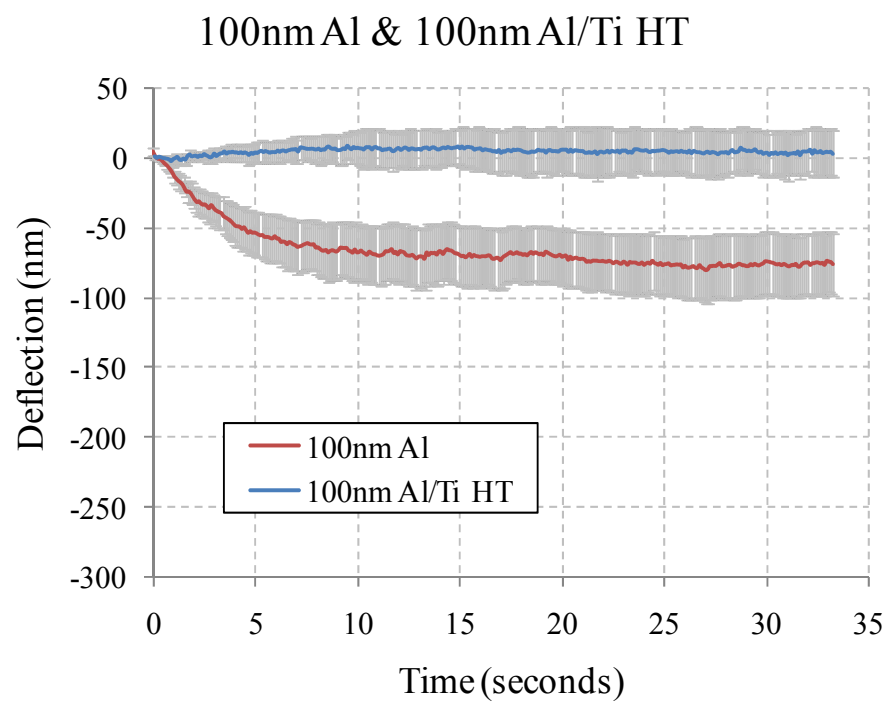

Figure 35. 100nm Al film compared to the 100nm Al-Ti film. 
Figure 36 displays the results from the $150 \mathrm{~nm} \mathrm{Al}$ and AlTi thin films. A viscoelastic response was observed in the $150 \mathrm{~nm}$ AlTi film; however, this response was reduced by $85.8 \%$ as compared to the aluminum thin film.

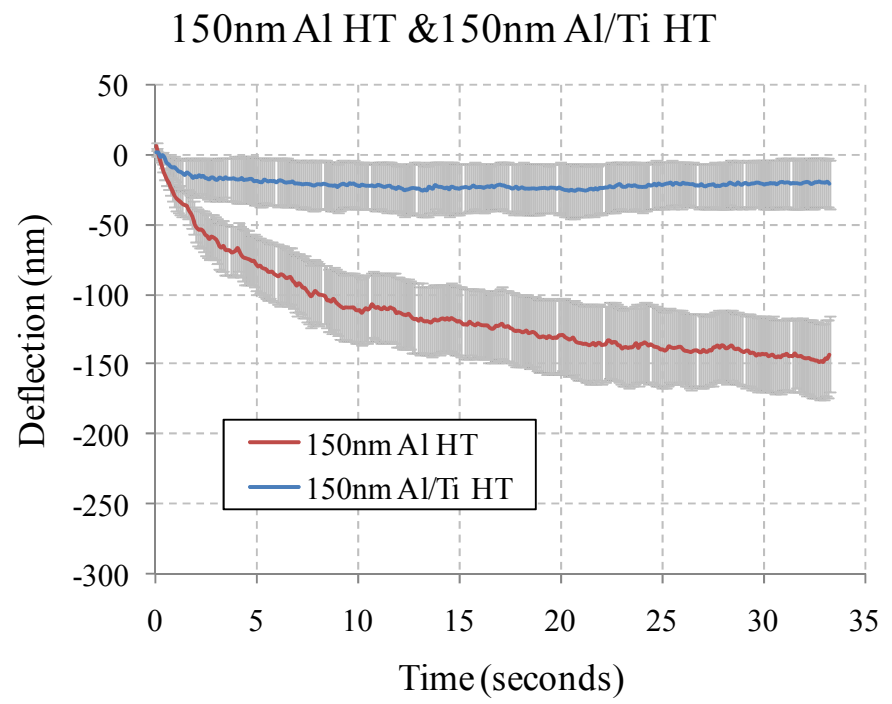

Figure 36. Comparison of the $150 \mathrm{~nm} \mathrm{Al} \mathrm{film} \mathrm{and} \mathrm{the} 150 \mathrm{~nm}$ Al-Ti film showing a reduction in viscoelastic response.

Table VII summarizes the effect of adding the titanium to the aluminum thin films. In both cases the viscoelastic response was reduced. The verification of the precipitate formation could not be accomplished with existing characterization techniques available.

Table VII. Results From Al Thin Films Compared To Alti Thin Films

\begin{tabular}{|l|r|r||l|r|r|}
\hline Film & Deflection & StdDev & Film & Deflection & StdDev \\
\hline $100 \mathrm{~nm} \mathrm{Al}$ & -76.43 & 22.06 & $150 \mathrm{~nm} \mathrm{Al}$ & -144.14 & 26.40 \\
\hline $100 \mathrm{~nm} \mathrm{A1} / \mathrm{Ti}$ & 3.23 & 16.04 & $150 \mathrm{~nm} \mathrm{Al} / \mathrm{Ti}$ & -20.48 & 17.33 \\
\hline Percent Decrease & $\sim 100 \%$ & & Percent Decrease & 85.8 & \\
\hline
\end{tabular}


The observation of the viscoelastic response in both the gold and aluminum thin films identifies the mechanical element of the devices as the source of this phenomenon. This is further supported by the reduction of the viscoelastic response observed in the AlTi thin films where the addition of titanium acted to reduce the viscoelastic response. While the direct measurement of defect diffusion along grain boundaries or dislocation bowing within the crystal lattice is beyond the scope of this study, the reduction of the viscoelastic response observed in the AlTi thin films strongly suggests that defect motion is the fundamental cause of this phenomenon. The verification of precipitate formation could have been accomplished through electrical resistivity measurements of the AlTi thin film before and after heat treatments. This data was not collected, however. It is expected that the resistivity of the thin films will increase as a result of precipitation formation. Increasing the at $\%$ of $\mathrm{Ti}$ in the aluminum thin films will allow for additional precipitates to form. This would further inhibit defect motion and could eliminate the viscoelastic response observed in the $150 \mathrm{~nm}$ thick AlTi films. An alloying element for gold thin films was not investigated; however, experiments relating to stress relaxation in RF micro switches indicate that 5 at $\%$ vanadium acts to impede defect motion in gold thin films (12). 


\section{Chapter 8 Future work}

The viscoelastic response measurements were limited by the amount of background noise and drift over time associated with the profilometer. Ultimately, this created a time boundary of 33 seconds past which any additional viscoelastic response was not detectable. The measurement capability of the RTS is substantially better with the ability to resolve $0.003^{\circ}$ over a 50 minute time period as compared to the profilometer with $0.011^{\circ}$ over a 33 second time period. The next step is to design a electrostatically actuated test device that can be evaluated using the RTS to measure the viscoelastic response. While the addition of titanium to the aluminum thin films showed a significant reduction of the viscoelastic response, a suitable alloying element for gold thin films was not accomplished with this study. Vanadium has been shown to reduce stress relaxation in gold films in the application of RF micro switches at room temperatures. Since gold is a common thin film used in microsystems an $\mathrm{Au} / \mathrm{V}$ thin film should be studied to identify its potential to reduce or eliminate the viscoelastic response seen in the gold films.

In addition to the increased resolution of the RTS, it is also capable of temperature control to within $\pm 0.35^{\circ} \mathrm{C}$. Additional studies of the viscoelastic response of Al-Ti films at temperatures up to $90^{\circ} \mathrm{C}$ could be conducted to identify any temperature dependence associated with the viscoelastic response.

Considerable effort was made to characterize the grain structure of the thin films using electron backscatter diffraction (EBSD). Due to the grain size of the films, $100 \mathrm{~nm}$, the EBSD system was unable to provide repeatable data. By increasing the grain size to a 
minimum diameter of $500 \mathrm{~nm}$ either through post sputtering heat treatment or by heating the substrate during deposition, additional studies of the effect of grain size on the viscoelastic response could be combined with grain orientation and size analysis using the EBSD system. 


\section{References}

1. Liu, Chang. Foundations of MEMS. New Jersey : Pearson Prentice Hall, 2006.

2. Lakes, Roderic S. Viscoelastic Solids. Boca Raton, FL : CRC Press LLC, 1998.

3. Elastic and Anelastic Behavior of Materials in Small Dimensions. Baker, Shefford P., P.Vinci, Richard and Arias, Tomás. 2002, Materials Research Society, pp. 26-29.

4. DMD reliability: a MEMS success story. Douglass, Michael R. Plano : Society of Photo-Optical Instrumentation Engineers Proceedings, 2003, Vol. 4980.

5. Linear viscoelasticity in aluminum thin films. Seungmin Hyun, Tejpal K. Hooghan, Walter L. Brown, and Richard P. Vinci. Bethlehem : Applied Physics Letters, 2005, Vol. 87.

6. Free Space Path Loss of UWB Communications. Pichaya Supanakoon, Sathit Aroonpraparat, Sathaporn Promwong and Jun-ichi Takada. Tokyo : Tokyo Institute of Technology, 2005.

7. Huigens, Brent. Mechanical Reliability of Micro Electrostatic Actuation. San Luis Obispo : California Polytechnic State University, 2007.

8. Zener, Clarence. Elasticity and Anelasticity of Metals. Chicago : The University of Chicago Press, 1948.

9. Berry, A. S. Nowick and B. S. Anelastic Relaxation in Crystalline Solids. New York and London : Academic Press, 1972.

10. Anelastic behavior of copper thin films on silicon substrates: Damping associated with dislocations. Nix, Dae-han Choi and William D. 679-687, s.l. : Acta Materialia, 2006, Vol. 54.

11. Nucleation and Precipitation Strengthening in Dilute Al-Ti and Al-Zr Alloys. Keith E. Knipling, David C. Dunand, and David N. Seidman. 2007, METALLURGICAL AND MATERIALS TRANSACTIONS A, VOLUME 38A, pp. 2552-2563.

12. Temperature-dependent microtensile testing of thin film materials for application to microelectromechanical system. Ming-Tzer Lin, Paul El-Deiry, Richard R. Chromik, Nicholas Barbosa, Walter L. Brown, Terry J. Delph, Richard P. Vinci. 2006, Microsystem Technologies Vol 12, pp. 10451051. 


\section{Bibliography}

Alred, Brusaw, Oliu. Handbook of Technical Writing. New York : St. Martin's Press, 2003.

Elastic and Anelastic Behavior of Materials in Small Dimensions. Baker, Shefford P., P.Vinci, Richard and Arias, Tomás. 2002, Materials Research Society, pp. 26-29.

Berry, A. S. Nowick and B. S. Anelastic Relaxation in Crystalline Solids. New York and London : Academic Press, 1972.

Bulk micromachining of silicon in TMAH-based etchants for aluminum passivation and smooth surface. Biswas, K., et al. 2006, Microelectronics Journal 37, pp. 321-327.

Etch characteristics of $\mathrm{KOH}, \mathrm{TMAH}$ and dual doped TMAH for bulk micromacbining of silicon.

Biswas, K., Kal, S. 2006, Microelectronics Journal 37, pp. 519-525.

Brown, and Richard P. Vinci. Bethlehem : Applied Physics Letters, 2005, Vol. 87.

Choi and William D. 679-687, s.l. : Acta Materialia, 2006, Vol. 54.

Anisotropic Etching CHaracteristics of Si in Tetramethylammonium Hydroxide : Isopropyl Alcohol : Pyrazine Solutions. Chung, Gwiy-Sang. 2005, Korean Physical Society Vol 46 No 5, pp. 1152-1156.

Nucleation and Precipitation Strengthening in Dilute Al-Ti and Al-Zr Alloys. Keith E. Knipling, David C. Dunand, and David N. Seidman. 2007, METALLURGICAL AND MATERIALS TRANSACTIONS A, VOLUME 38A, pp. 2552-2563.

Physical Origins of Intrinsic Stresses in Volmer-Weber Thin Films. Floro, Jerrold A., et al. 2002, Materials Research Society, pp. 19-25.

Huebner, Kenneth H., et al. The Finite Element Method for Engineers. New York: John Wiley \& Sons, Inc., 2001. 
Huigens, Brent. Mechanical Reliability of Micro Electrostatic Actuation. San Luis Obispo: California Polytechnic State University, 2007.

Diffusional Creep: Stresses and Strain Rates in Thin Films and Multilayers. Josell, D., Weihs, T.P. and Gao, H. 2002, Materials Research Society, pp. 39-44.

Lakes, Roderic S. Viscoelastic Solids. Boca Raton, FL : CRC Press LLC, 1998.

DMD reliability: a MEMS success story. Douglass, Michael R. Plano : Society of PhotoOptical Instrumentation Engineers Proceedings, 2003, Vol. 4980.

Liu, Chang. Foundations of MEMS. New Jersey : Pearson Prentice Hall, 2006.

Temperature-dependent microtensile testing of thin film materials for application to microelectromechanical system. Ming-Tzer Lin, Paul El-Deiry, Richard R. Chromik, Nicholas Barbosa, Walter L. Brown, Terry J. Delph, Richard P. Vinci. 2006, Microsystem Technologies Vol 12, pp. 1045-1051.

Ott, R. Lyman, Longnecker, Michael. Statistical Methods and Data Analysis 5th. Pacific Grove : Duxbury - Thomson Learning, 2001.

Phillips, Rob. Crystals, Defects and Microstructures Modeling Across Scales. Providence, RI : Cambridge University Press, 2004.

Linear viscoelasticity in aluminum thin films. Seungmin Hyun, Tejpal K. Hooghan, Walter L. Free Space Path Loss of UWB Communications. Pichaya Supanakoon, Sathit Aroonpraparat, Sathaporn Promwong and Jun-ichi Takada. Tokyo : Tokyo Institute of Technology, 2005.

Smooth etching of silicon using TMAH and isopropyl alcohol for MEMS applications. Sundaram, Kalpathy B., Vijayakumar, Arun and Subramanian, Ganesh. 2005, Microelectronic Engineering 77, pp. 230-241.

Young, Warren C. Roark's Formulas for Stress \& Strain 6th. New York : McGraw-Hill Book Company, 1989. 
Zener, Clarence. Elasticity and Anelasticity of Metals. Chicago : The University of Chicago Press, 1948. 
Appendix A. Lithography Masks

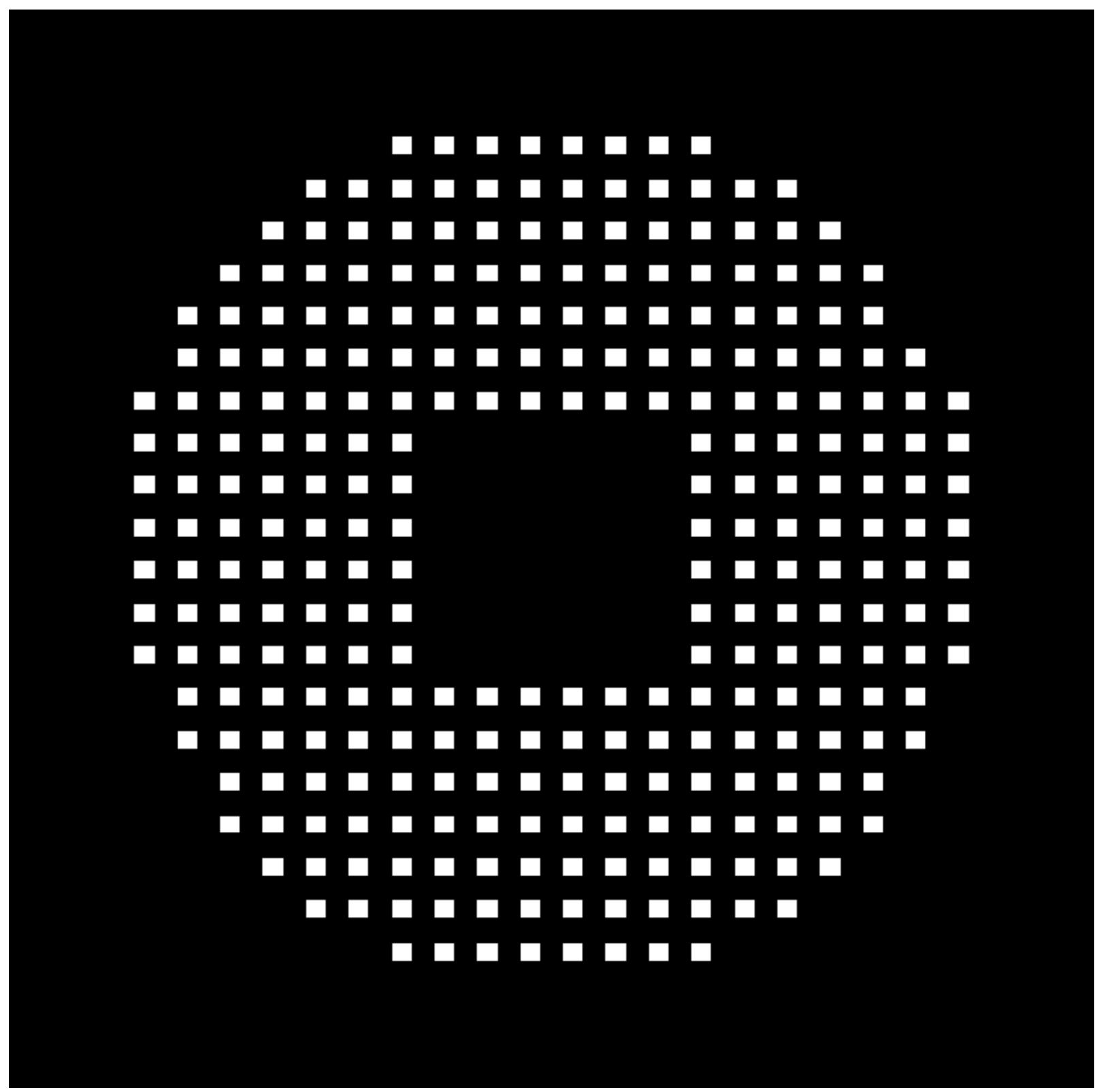

Figure 37 Mask 1 - Used to create the exposed silicon areas for deep etch wells on the back side of the wafer. 


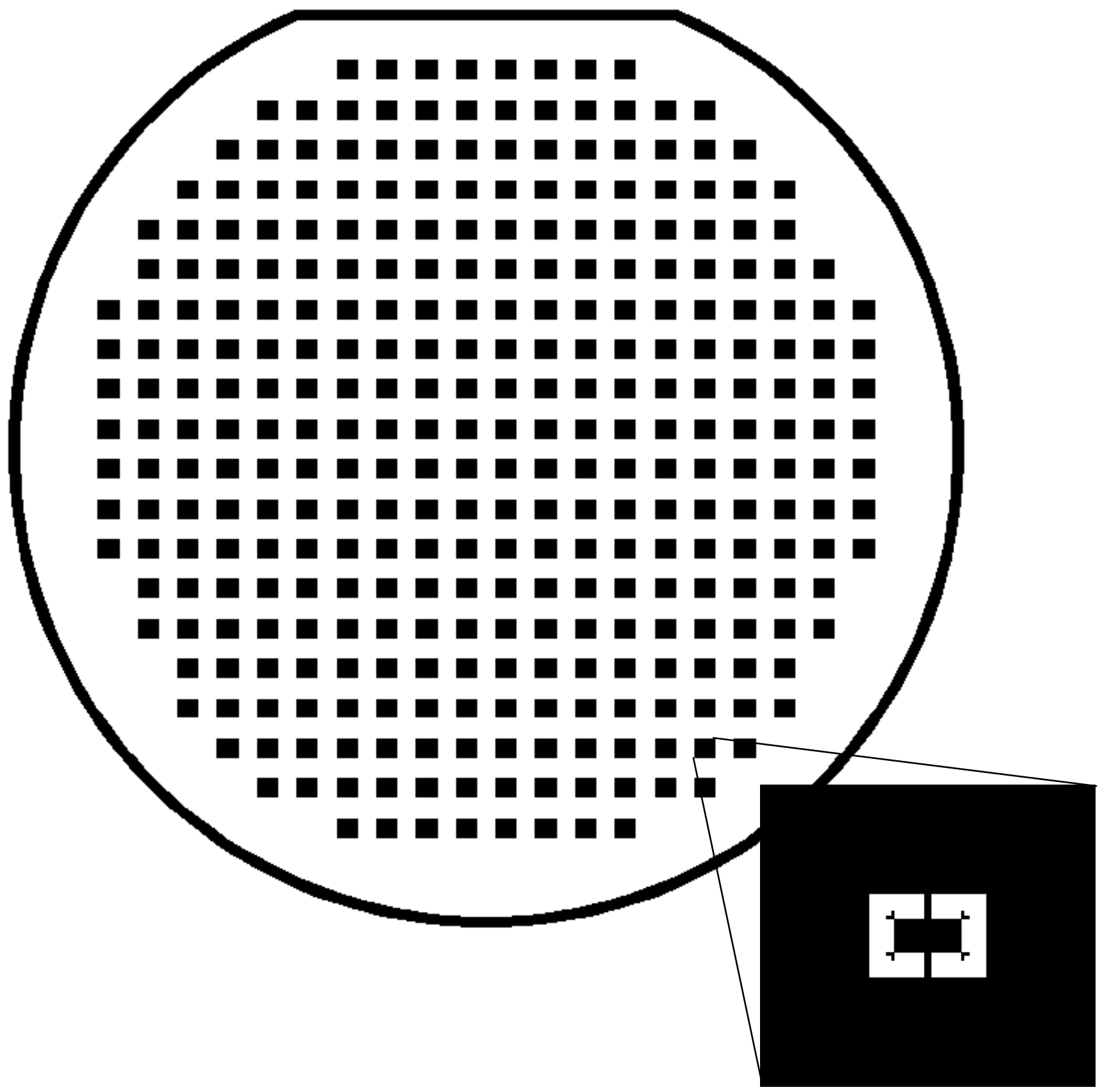

Figure 38 Mask 2 applied to top side of wafer to create the

exposed silicon areas for through etching. 


\section{Appendix B. Finite Element Analysis Data}

\section{Fixed-Fixed torsional hinge test device model}

Element type:

Linear quadrilateral, type S4R

Linear triangle, type S3R

Number of nodes: 93671

Number of elements: 93149

Element types: S3R S4

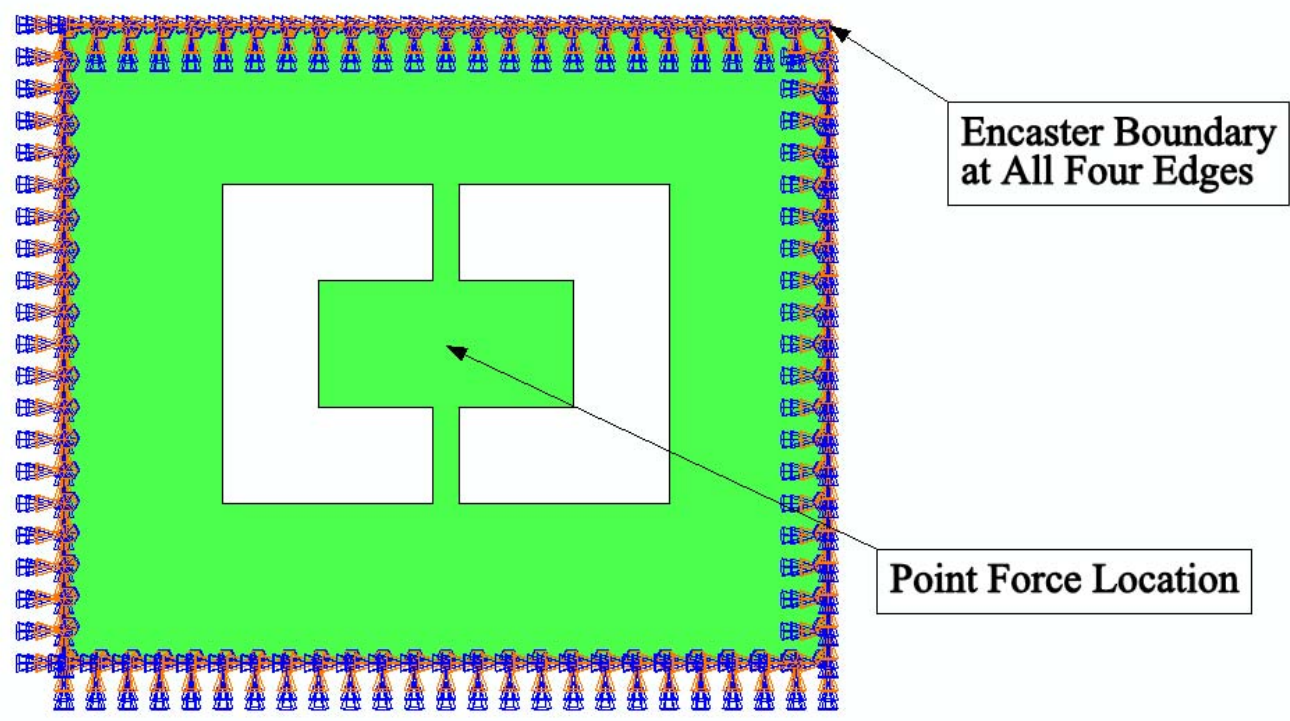

Figure 39. Boundary conditions applied to center deformation test device model. 


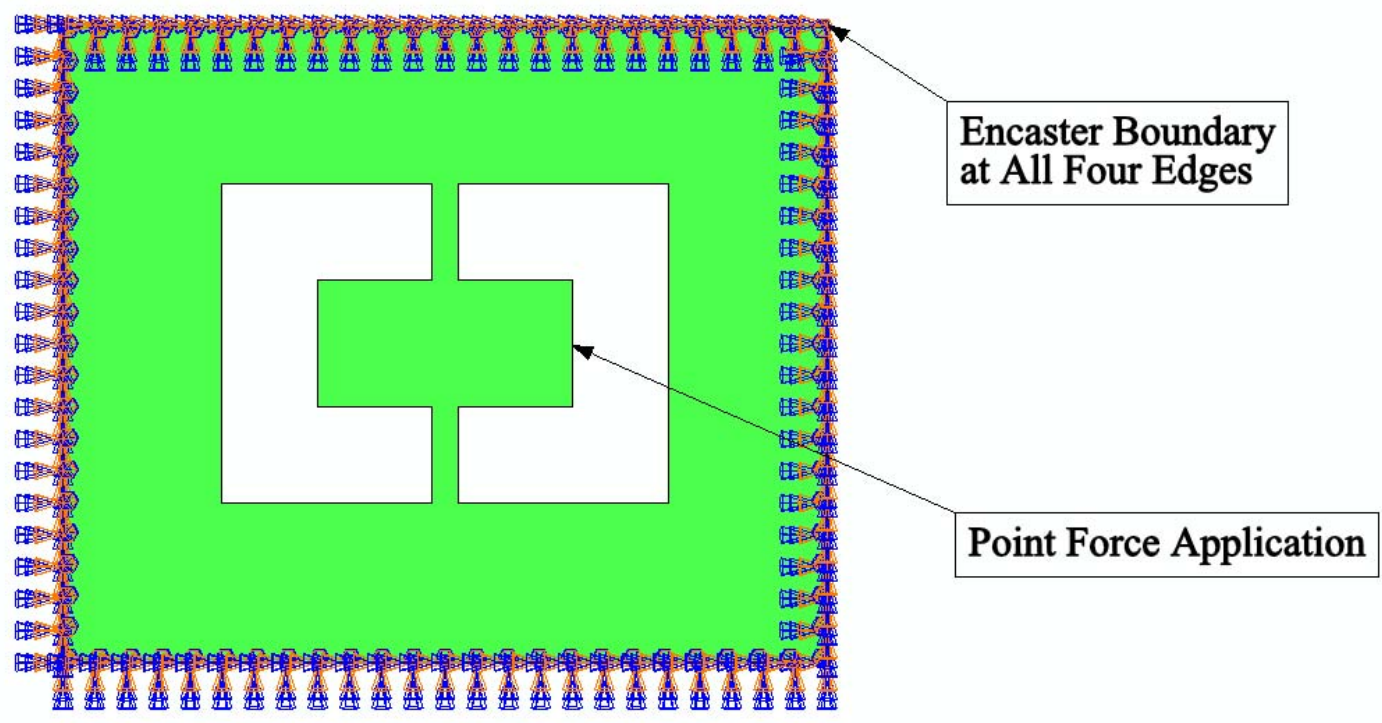

Figure 40. Boundary condition on torsional test device model.

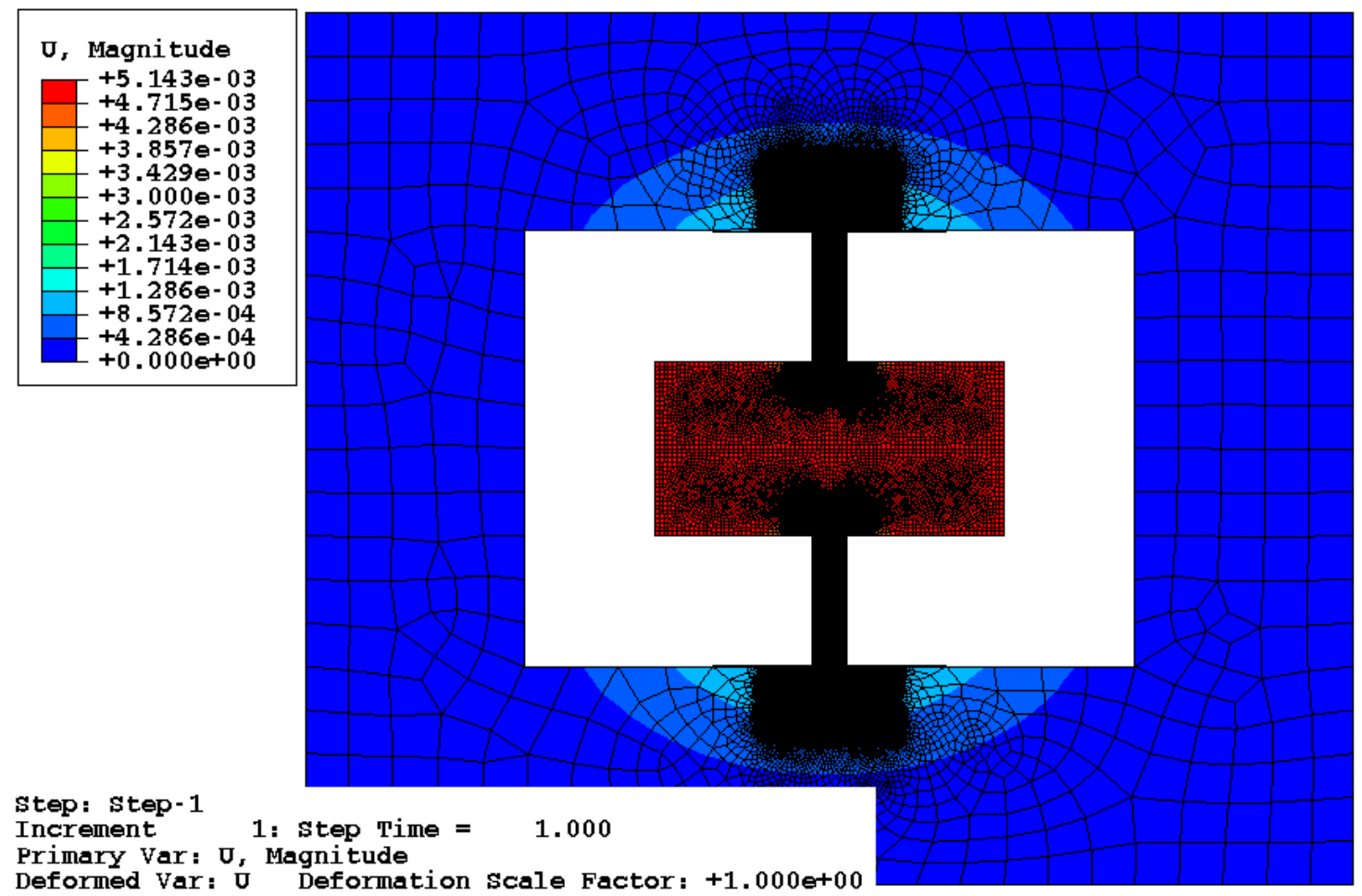

Figure 41. Deformation plot with applied load at center of device of the test device showing mesh density. 


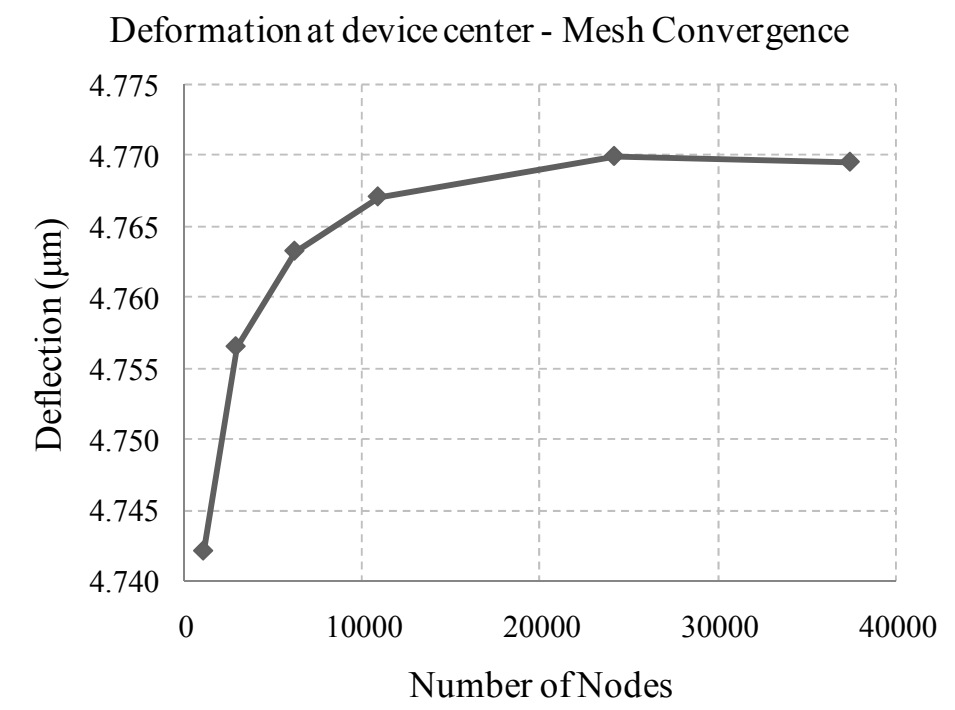

Figure 42. Mesh convergence of FEA model with applied force at the center of the device.

Table VIII. Comparison Of FEA Model To Hand Calculation And Measured Values

\begin{tabular}{|l|r|r|}
\hline & $\begin{array}{c}\text { Deflection at center } \\
\text { of device }(\mathrm{nm})\end{array}$ & $\begin{array}{c}\text { Percent Difference } \\
\text { from FEA }\end{array}$ \\
\hline FEA (nm) & 4769.8 & 4.58 \\
\hline Measured $(\mathrm{nm})$ & 4556.3 & 14.23 \\
\hline Calculation $(\mathrm{nm})$ & 4136.0 & \\
\hline
\end{tabular}

\section{Torsional Hinge Spring Constant}

The torsional spring constant is a function of the shear modulus of the materials used to create the actuators along with the physical dimensions of the spring.

$$
\begin{gathered}
\qquad \theta=\frac{T L}{J G} \\
\mathrm{~T}=\text { Applied moment }(\mathrm{Nm}) \\
L=\text { Length of actuator hinge } \\
\mathrm{J}=\text { Polar moment of inertia of the cross section of the hinge } \\
\mathrm{G}=\text { Modulus of rigidity (Shear modulus) } \\
\theta=\text { Angle of rotation in radians }
\end{gathered}
$$


For a rectangular cross section, the polar moment of inertia can be found using Equation 10.

$$
\begin{aligned}
J=a b^{3}\left[\frac{16}{3}-3.36 \frac{b}{a}\left(1-\frac{b^{4}}{12 a^{4}}\right)\right] \quad \text { for } a \geq b \\
2 \mathrm{a}=\text { hinge width } \\
2 \mathrm{~b}=\text { hinge thickness } \\
-2 \mathrm{a}-2 \mathrm{~b}
\end{aligned}
$$

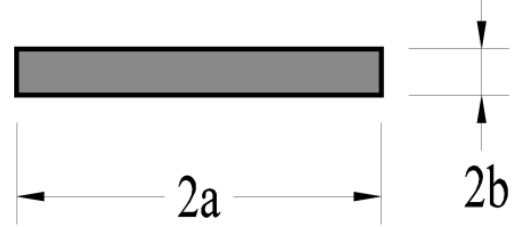

Figure 43. Cross section of torsional hinge.

Using Equations 9 and 10, the rotation of the actuator is calculated to be $5.33^{\circ}$ and is listed in Table IX. The FEA model predicts $5.37^{\circ}$ of rotation which compares well with the calculated value with less than $1 \%$ difference.

Table IX. Data Comparison Between Rotational FEA Model And Hand Calculation

\begin{tabular}{|l|r|c|}
\hline & $\begin{array}{c}\text { Rotation Deflection } \\
\text { (degrees) }\end{array}$ & $\begin{array}{c}\text { Percent Difference } \\
\text { from FEA }\end{array}$ \\
\hline FEA (nm) & 5.37 & \\
\hline Calculation $(\mathrm{nm})$ & 5.33 & 0.75 \\
\hline
\end{tabular}




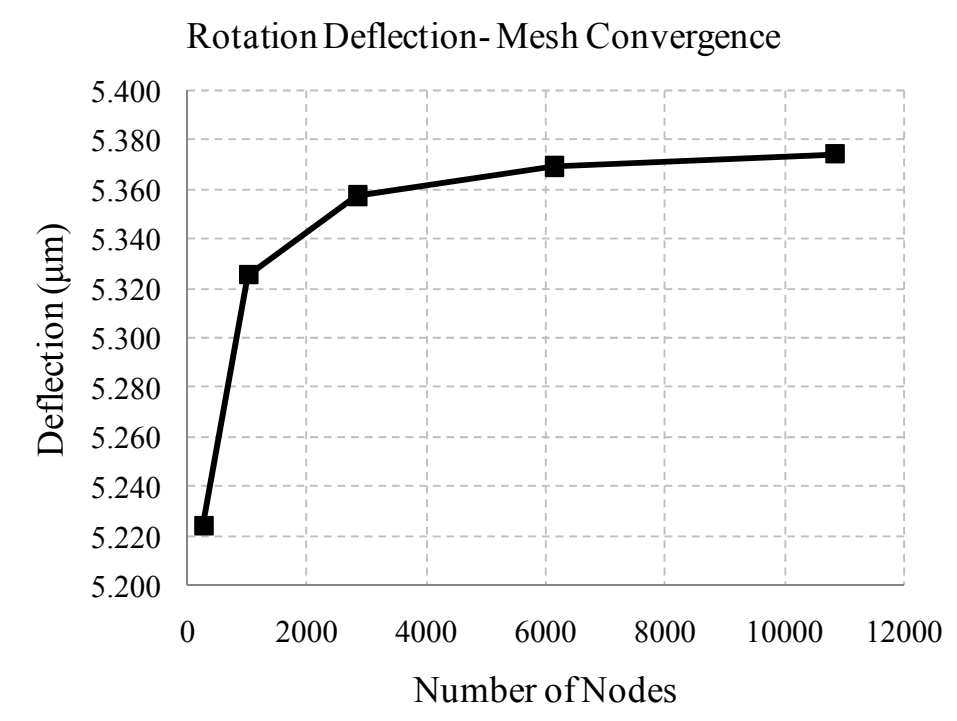

Figure 44. Mesh convergence of FEA model under a torsional load.

\section{Electrostatic actuator model}

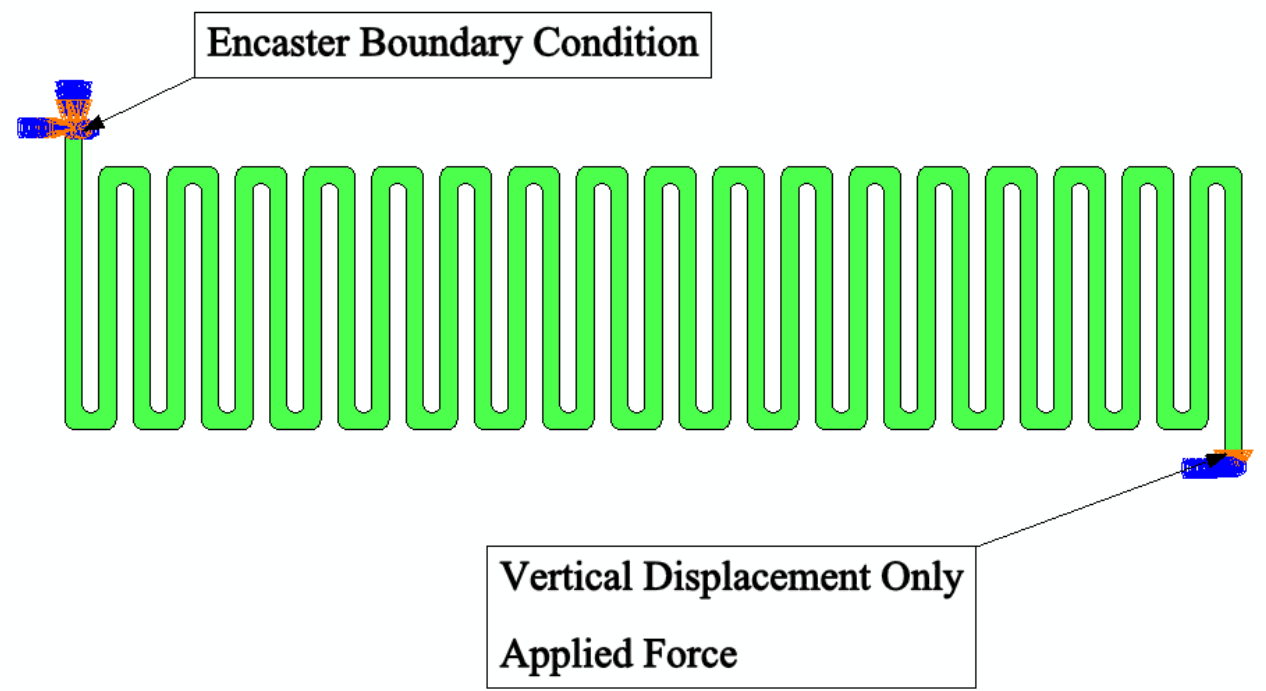

Figure 45. Boundary conditions applied to electrostatic model hinge.

Element type:

Linear quadrilateral, type S4R 
Linear triangle, type S3R

Number of nodes: 16469

Number of elements: 13968

13672 quadrilateral elements

296 triangular elements

Mesh Convergence - Electrostatic Actuator

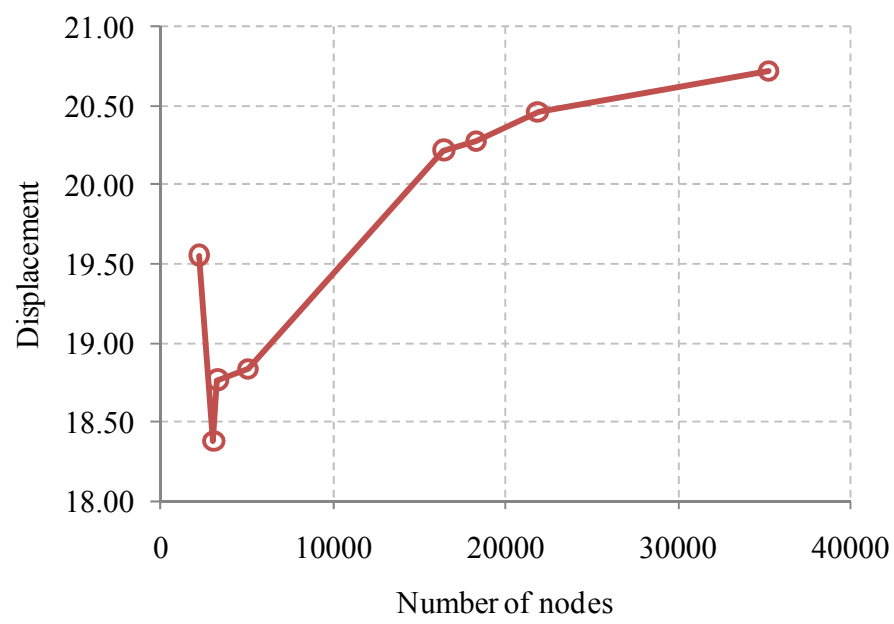

Figure 46. Mesh convergence for the electrostatic actuator model. 
Appendix C. Mechanical Drawings and RTS BOM

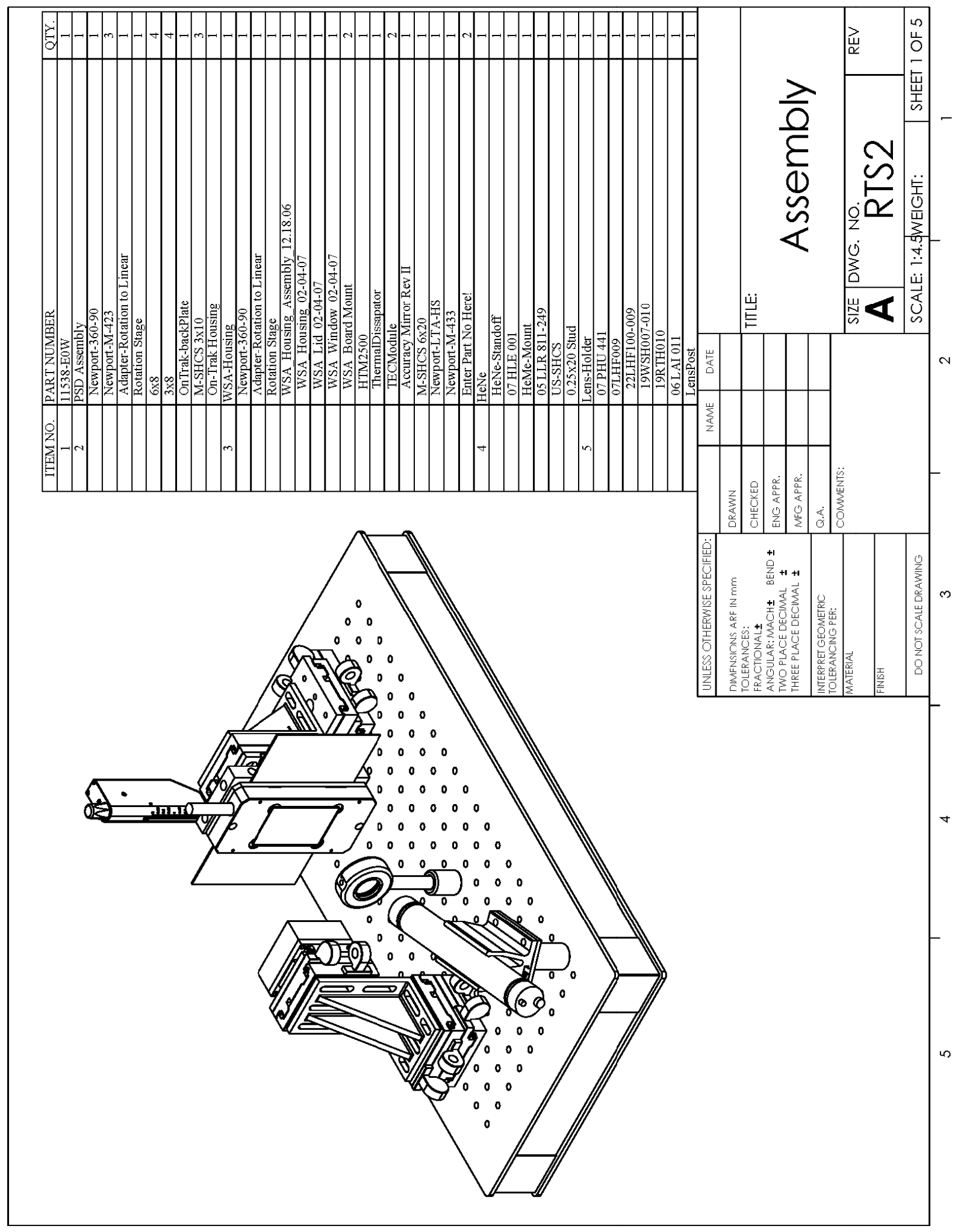

C-1 


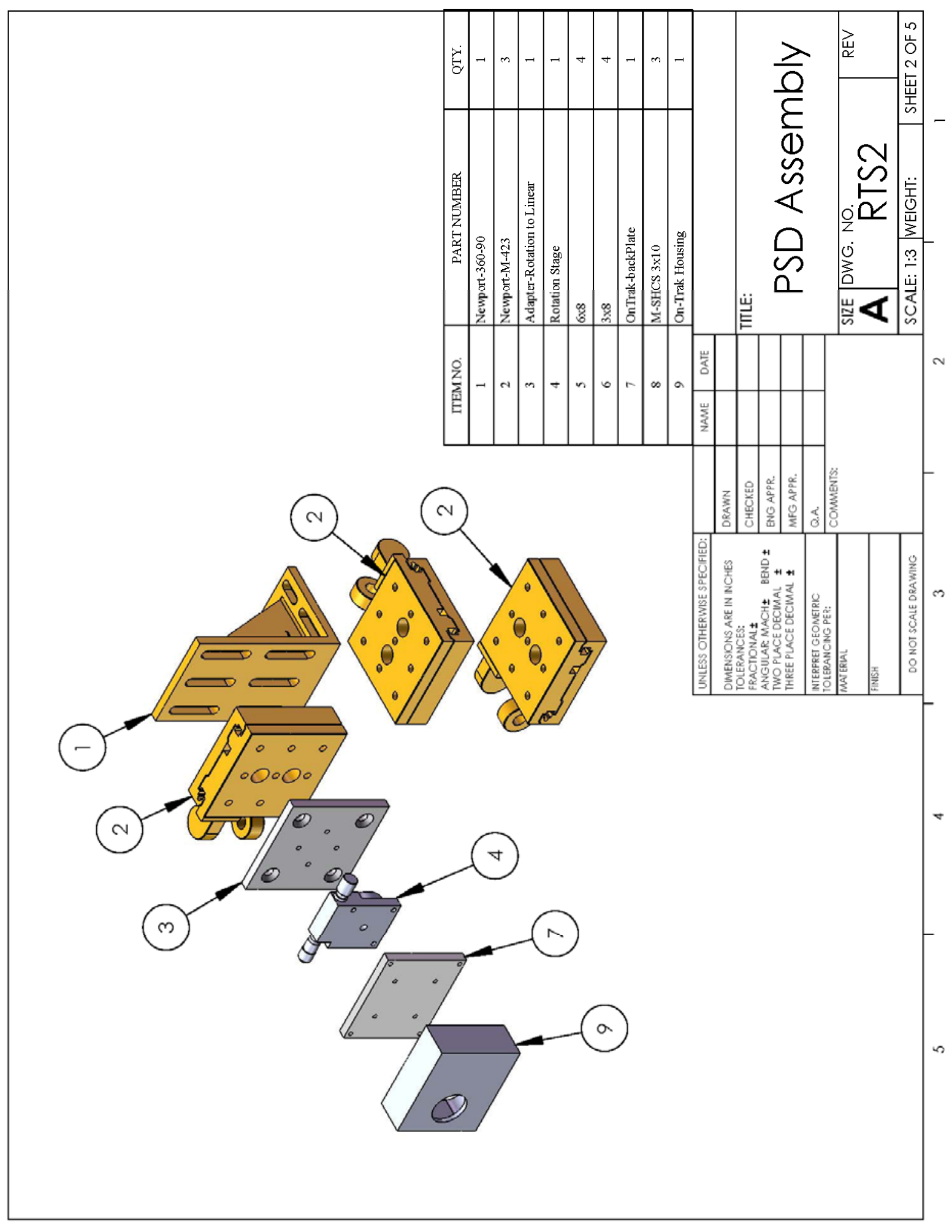

C-2 


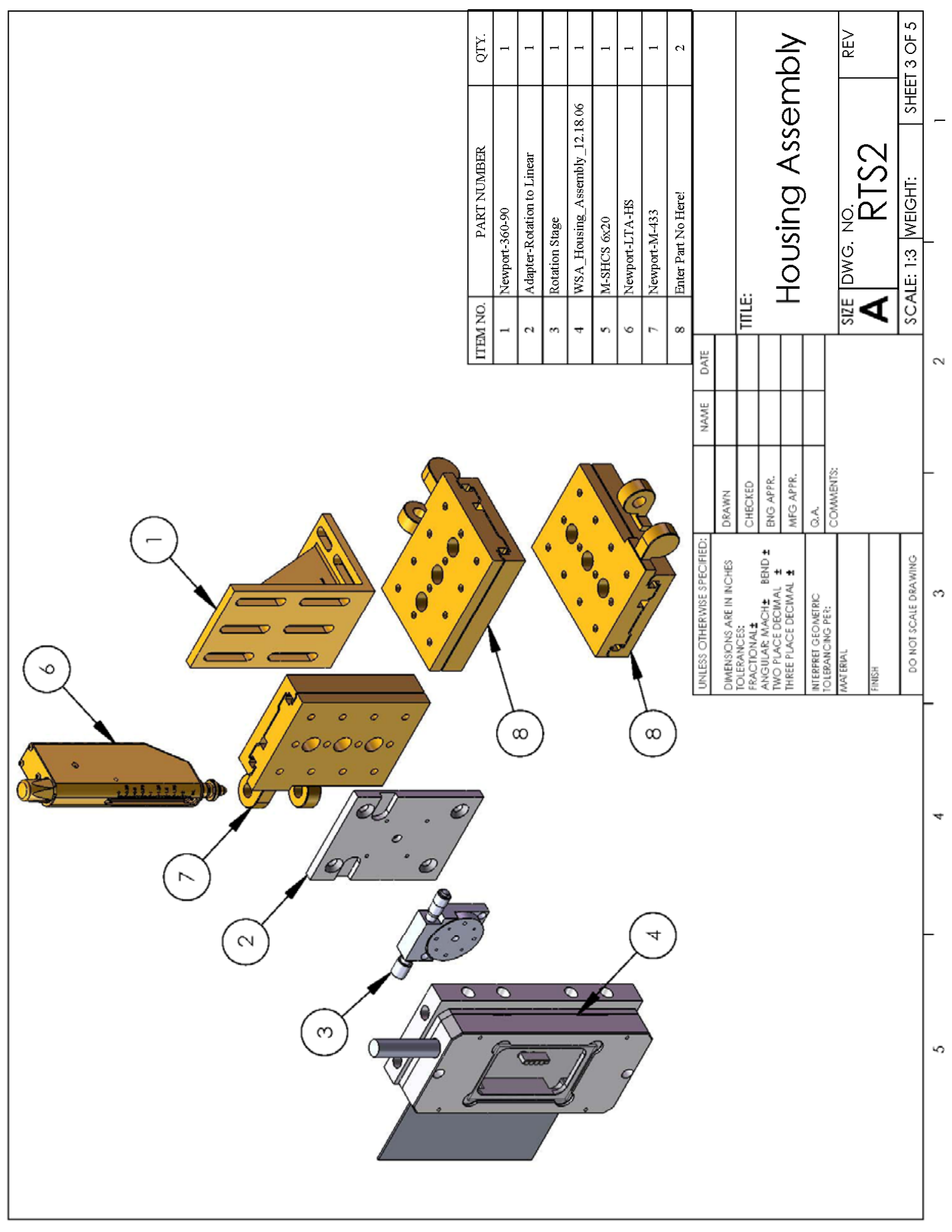

C-3 


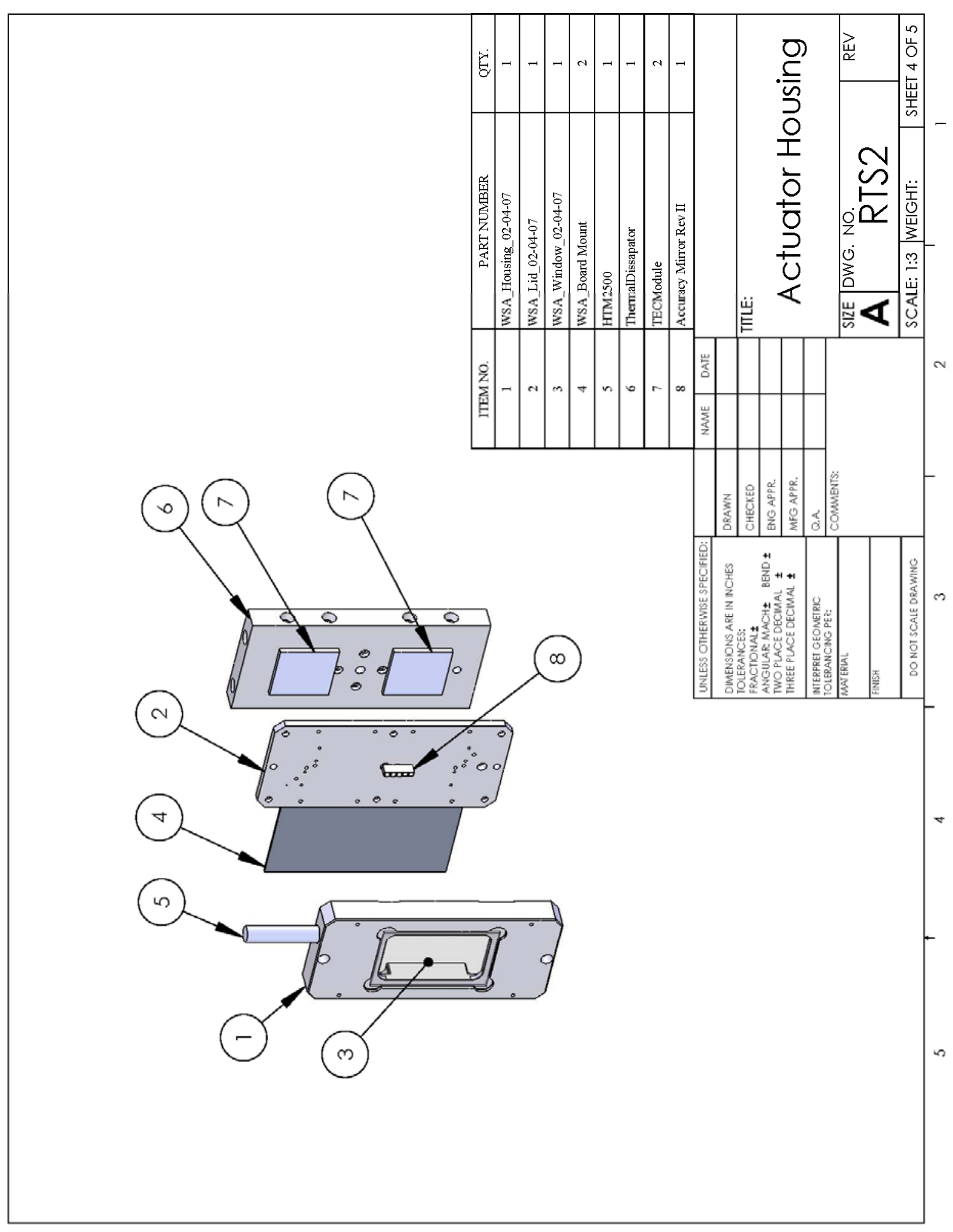

C-4 


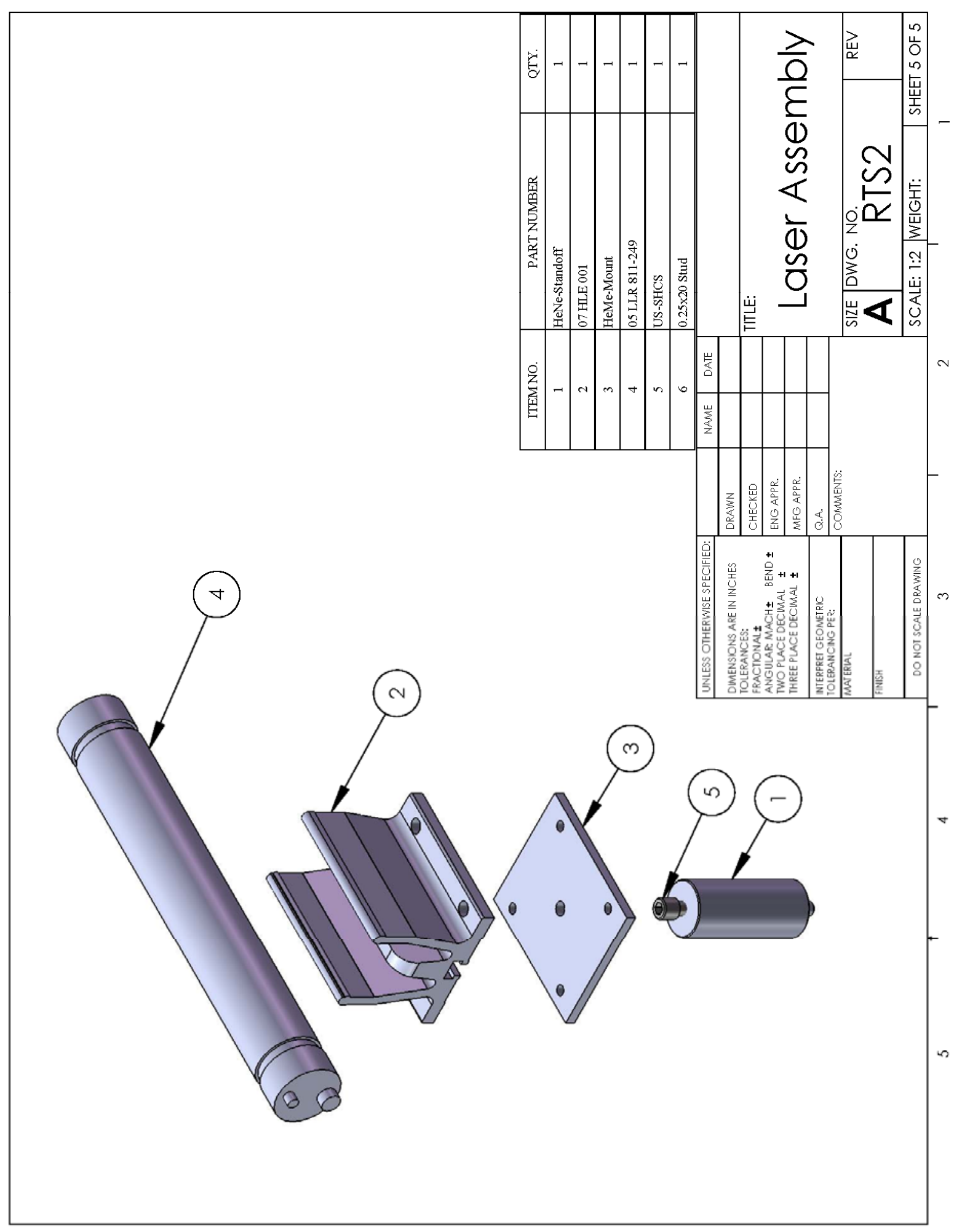

C-5 


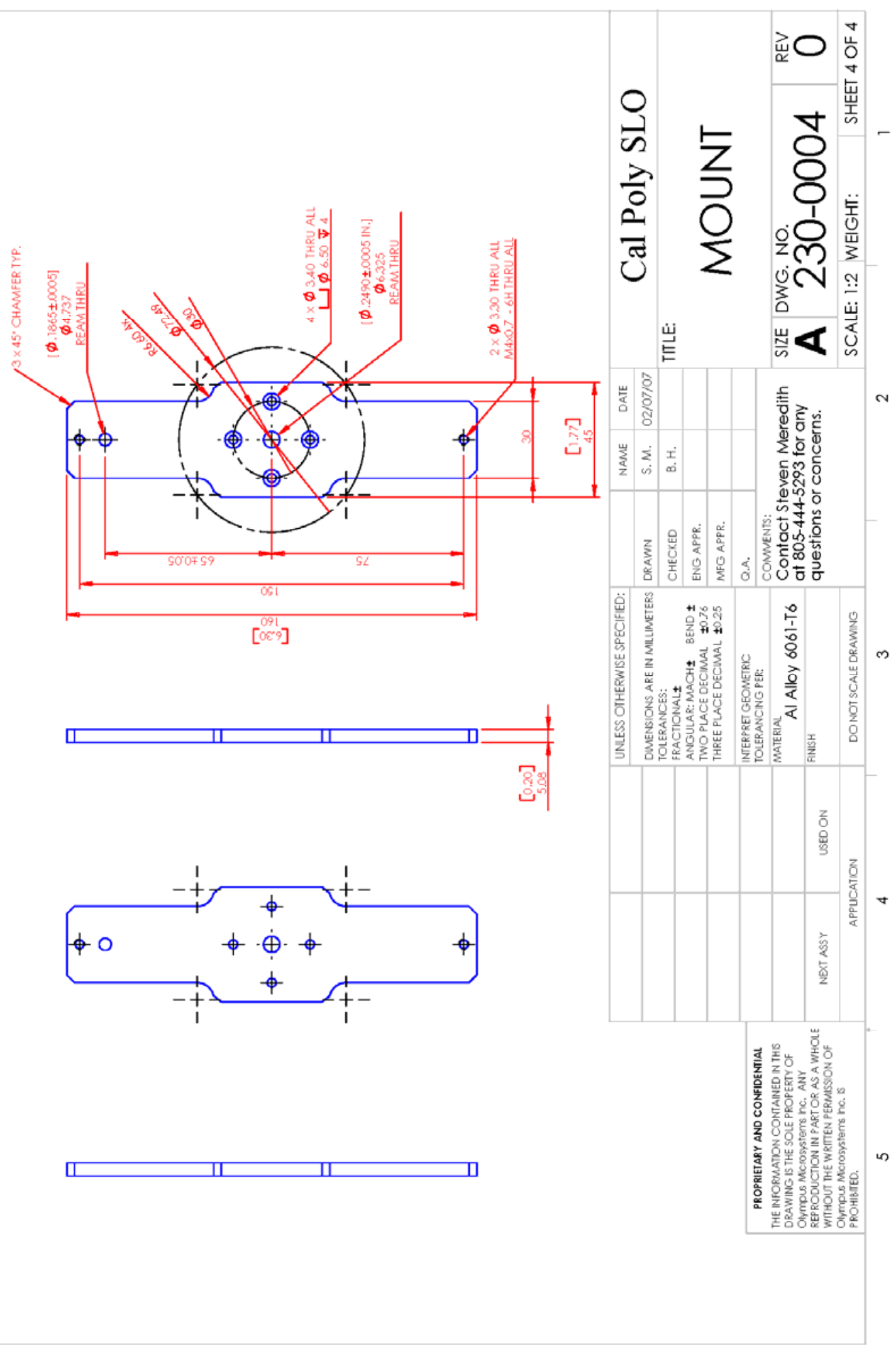

C-6 


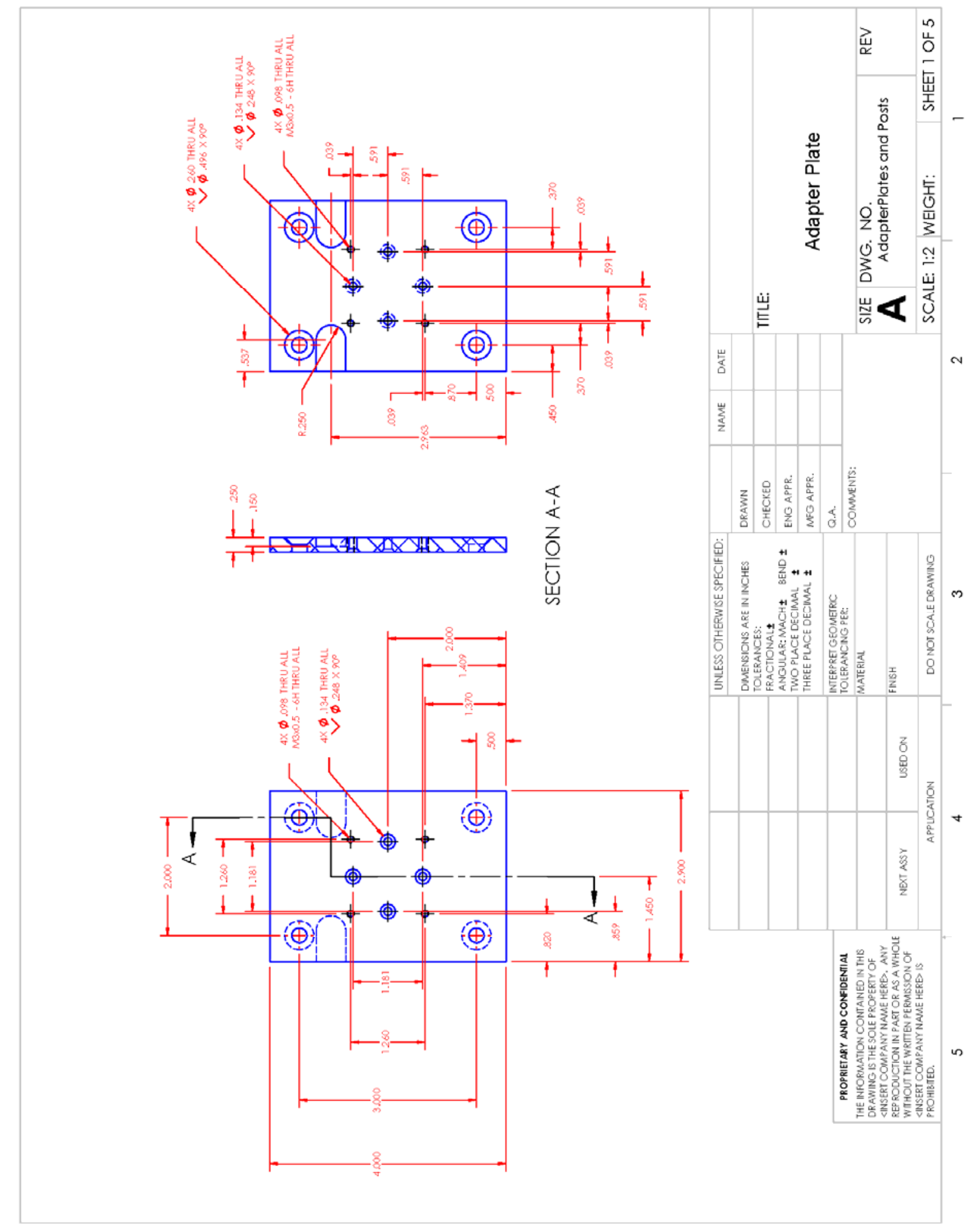

C-7 


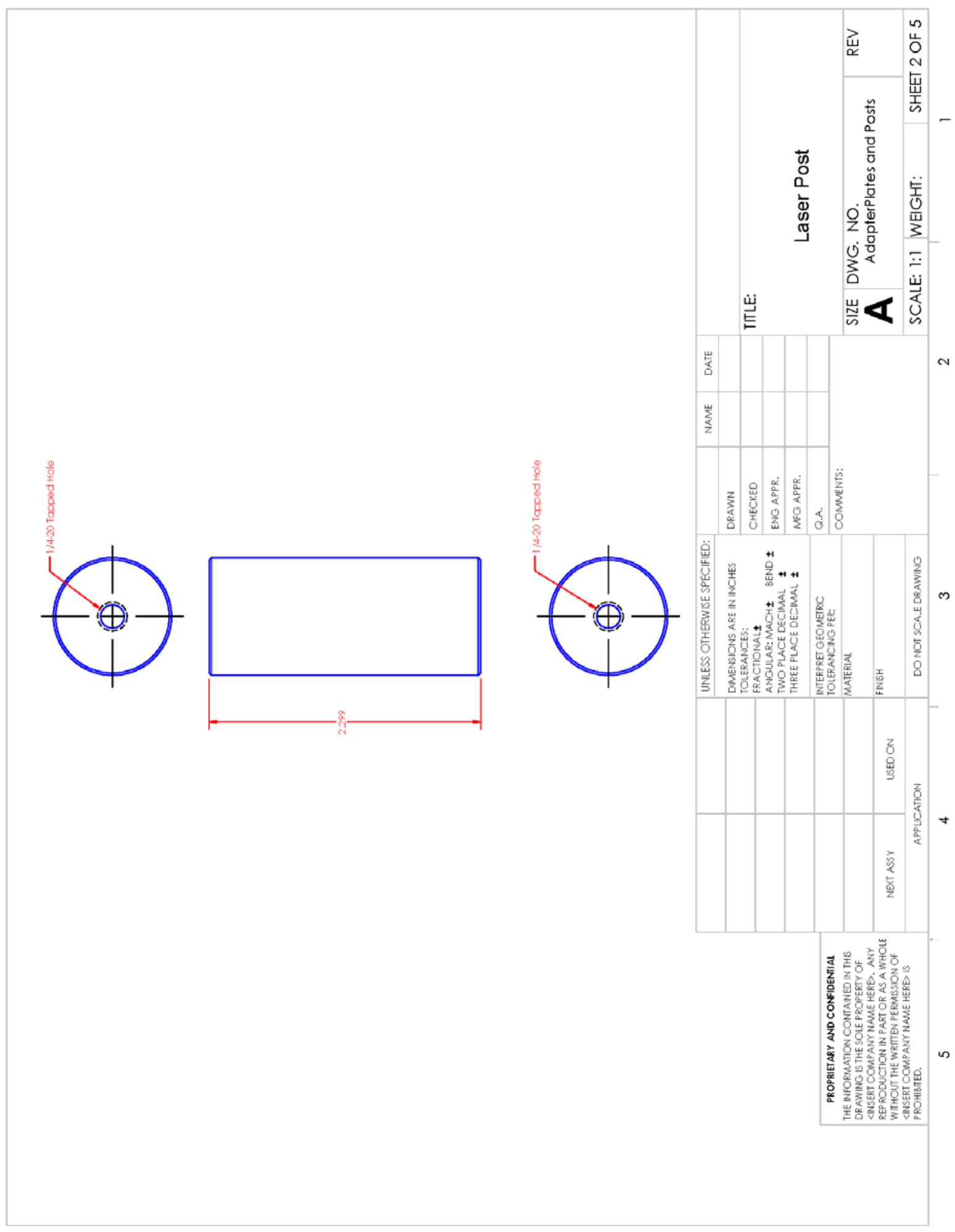

C-8 


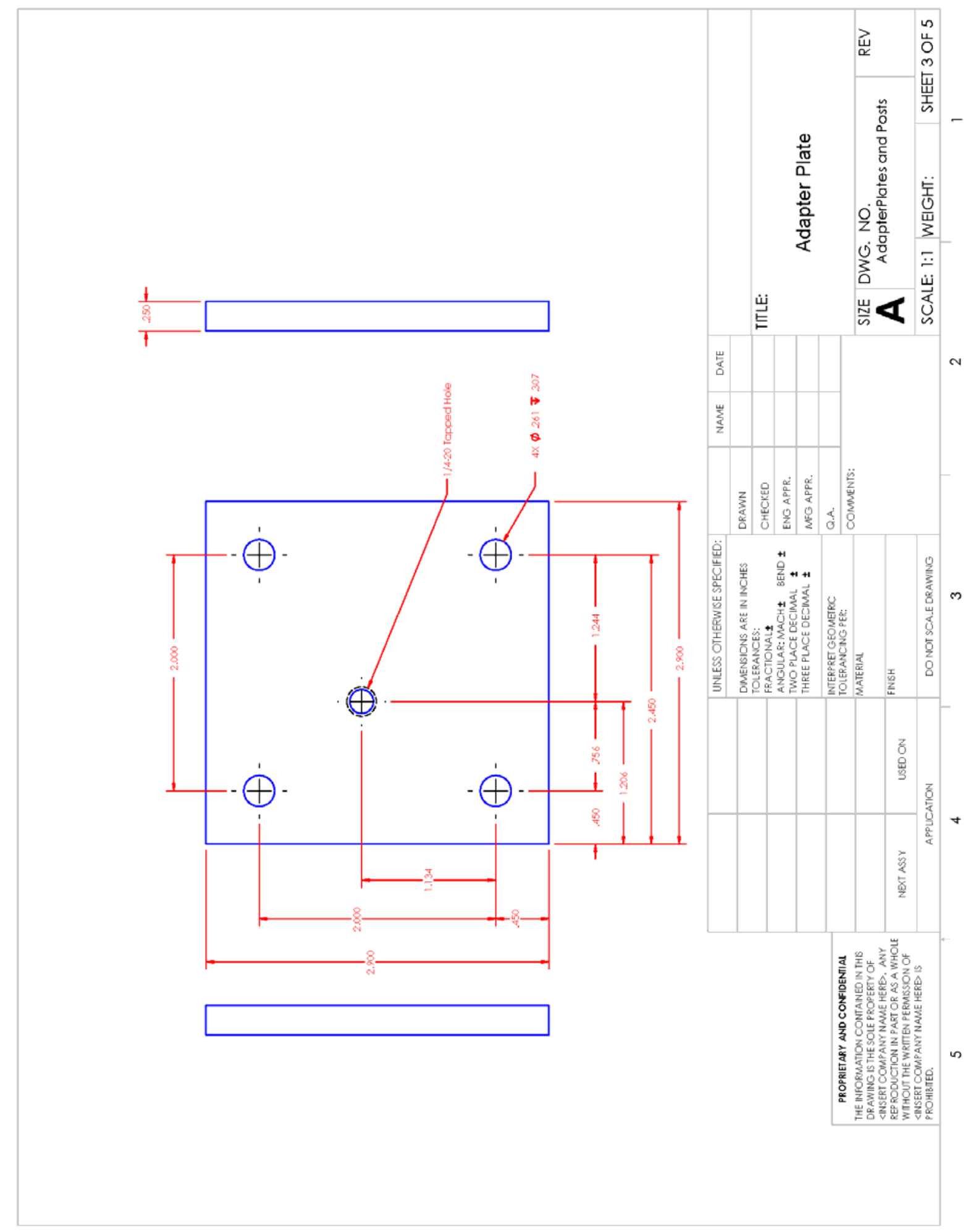

C-9 


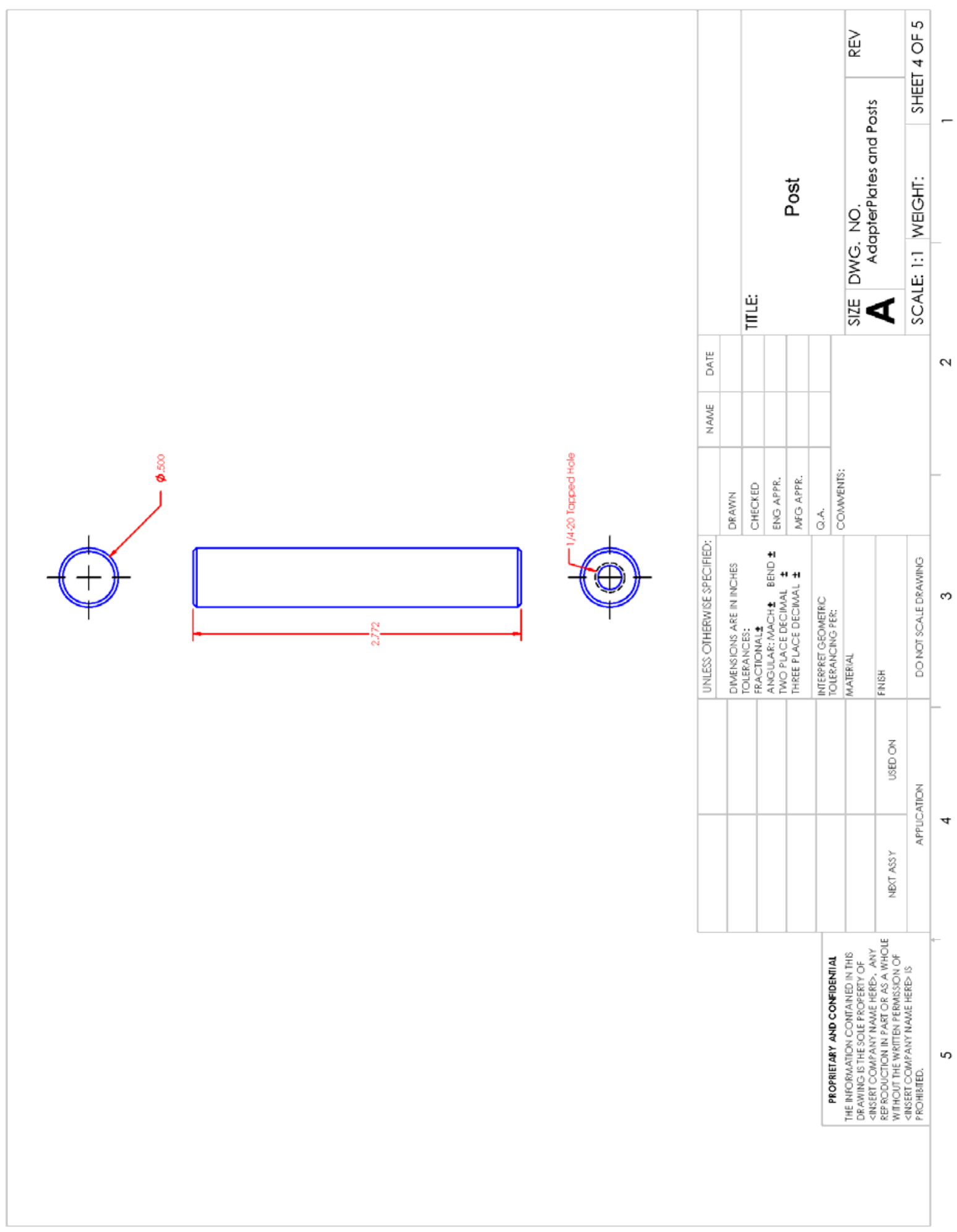

C-10 


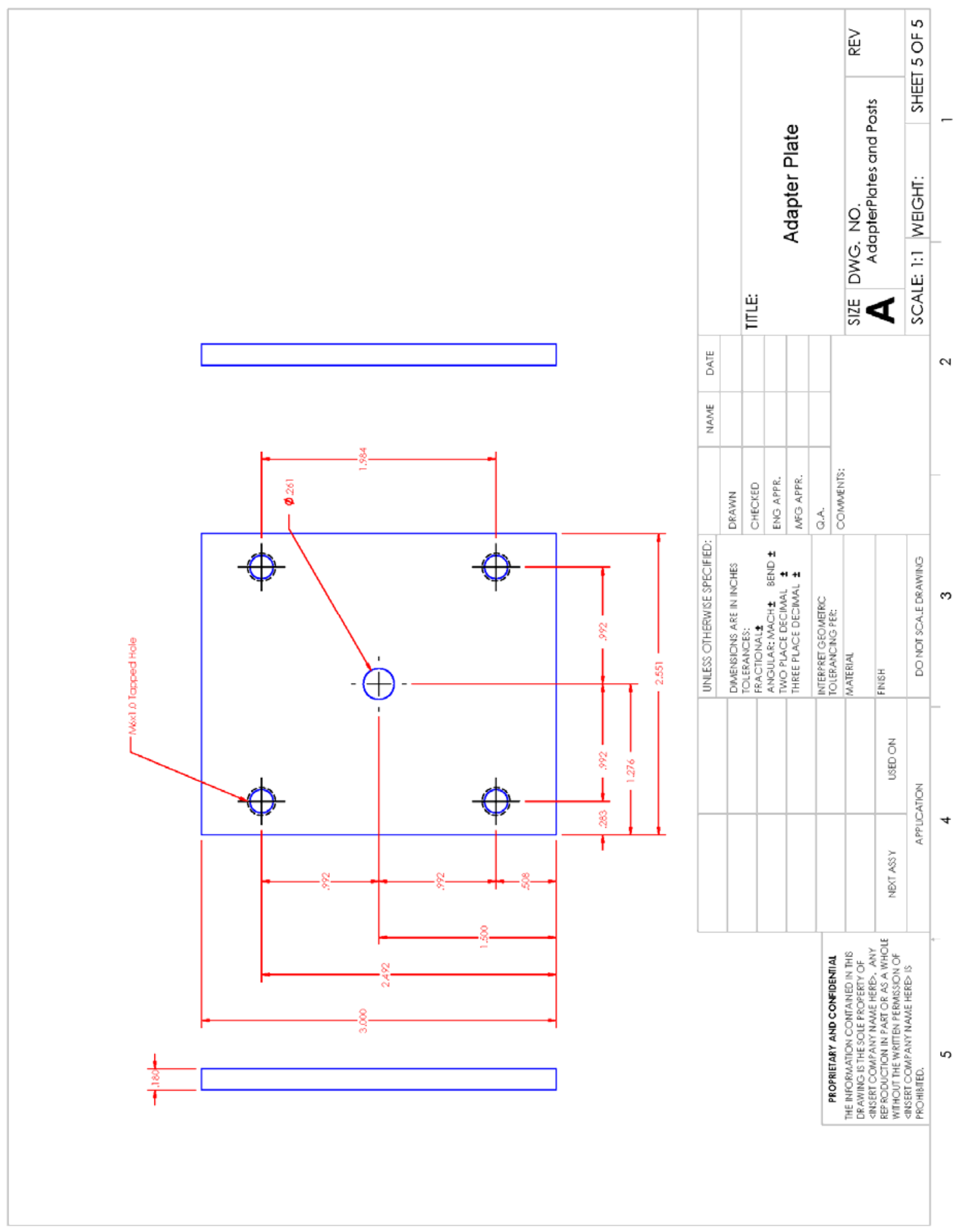

C-11 


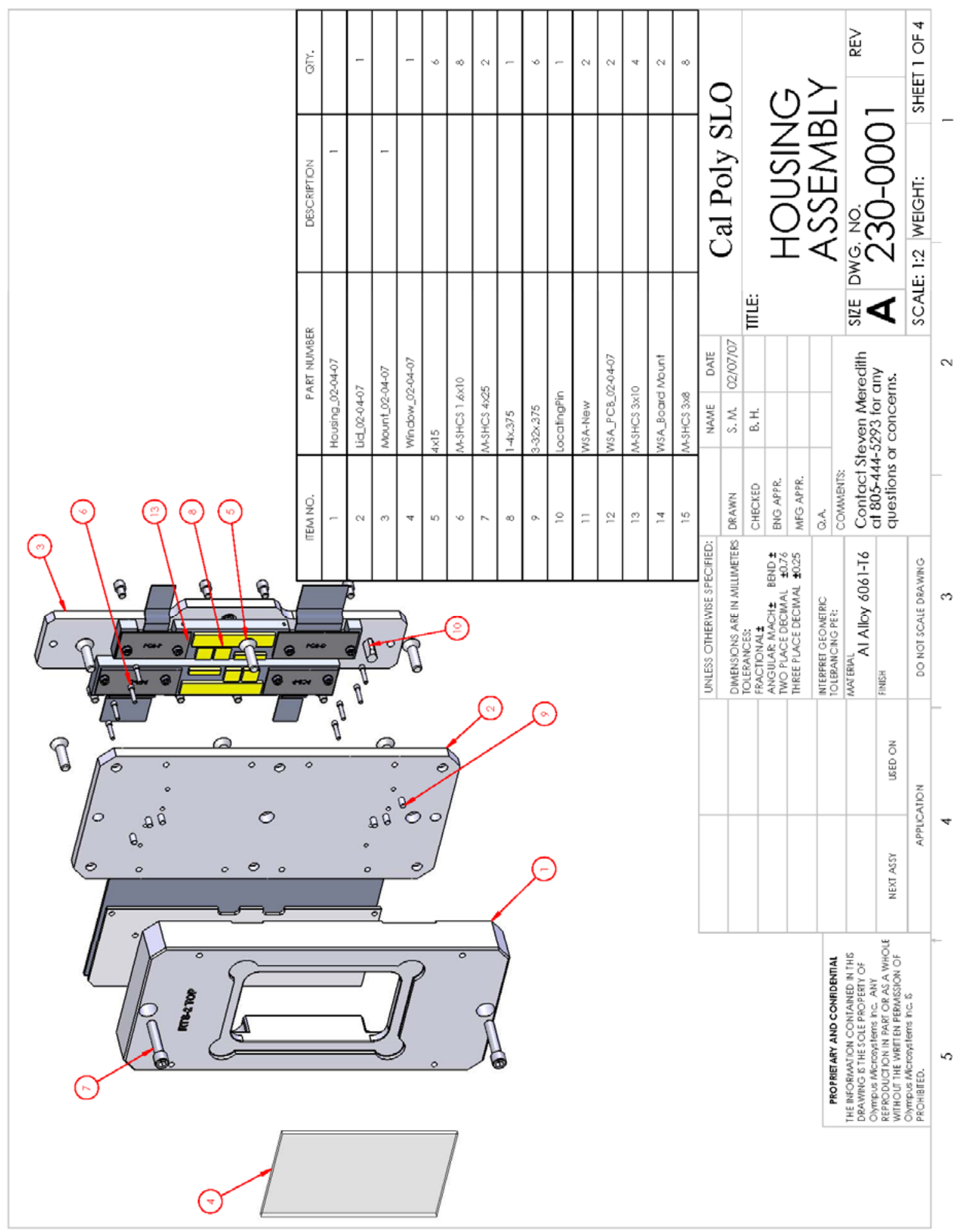

C-12 


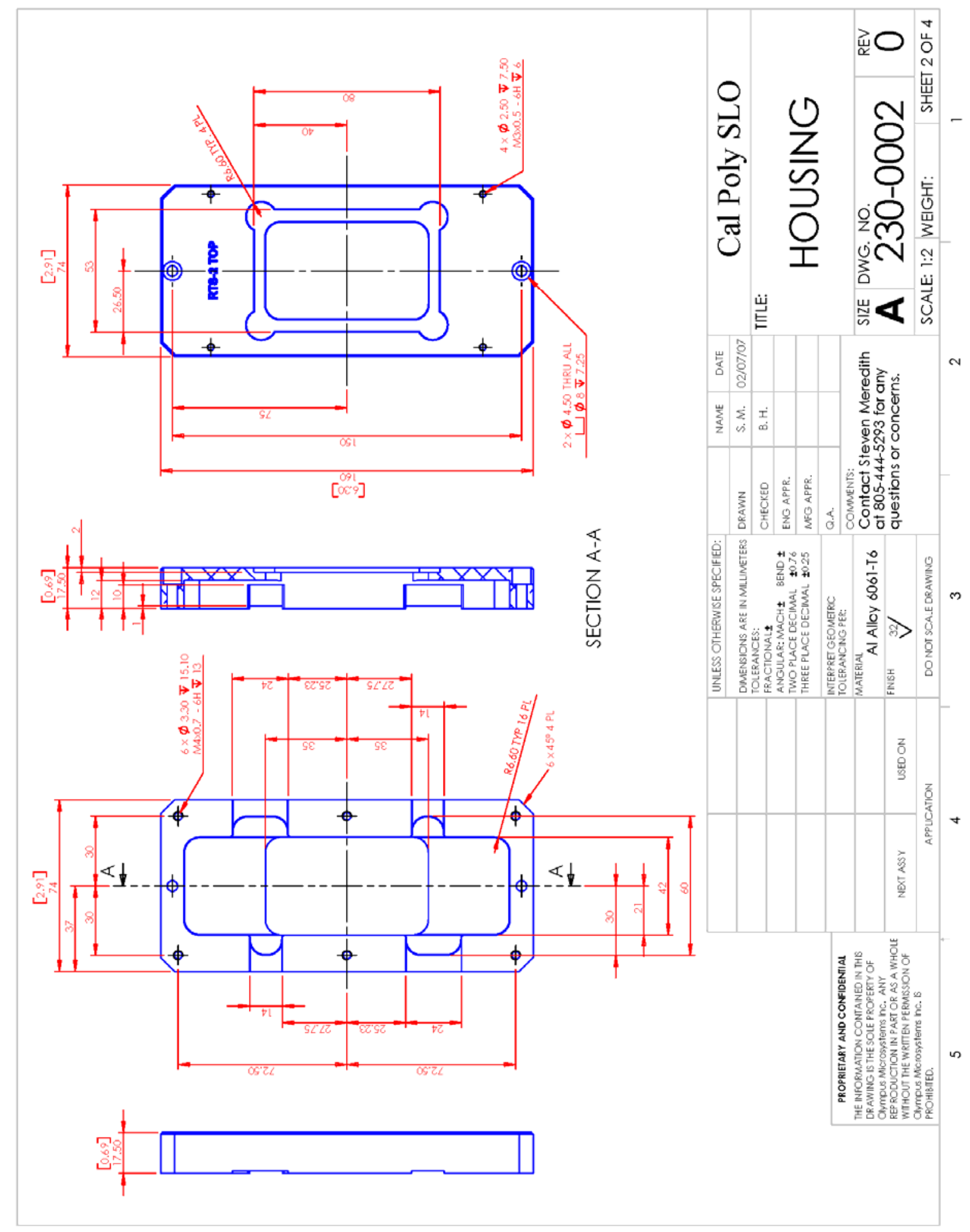

C-13 

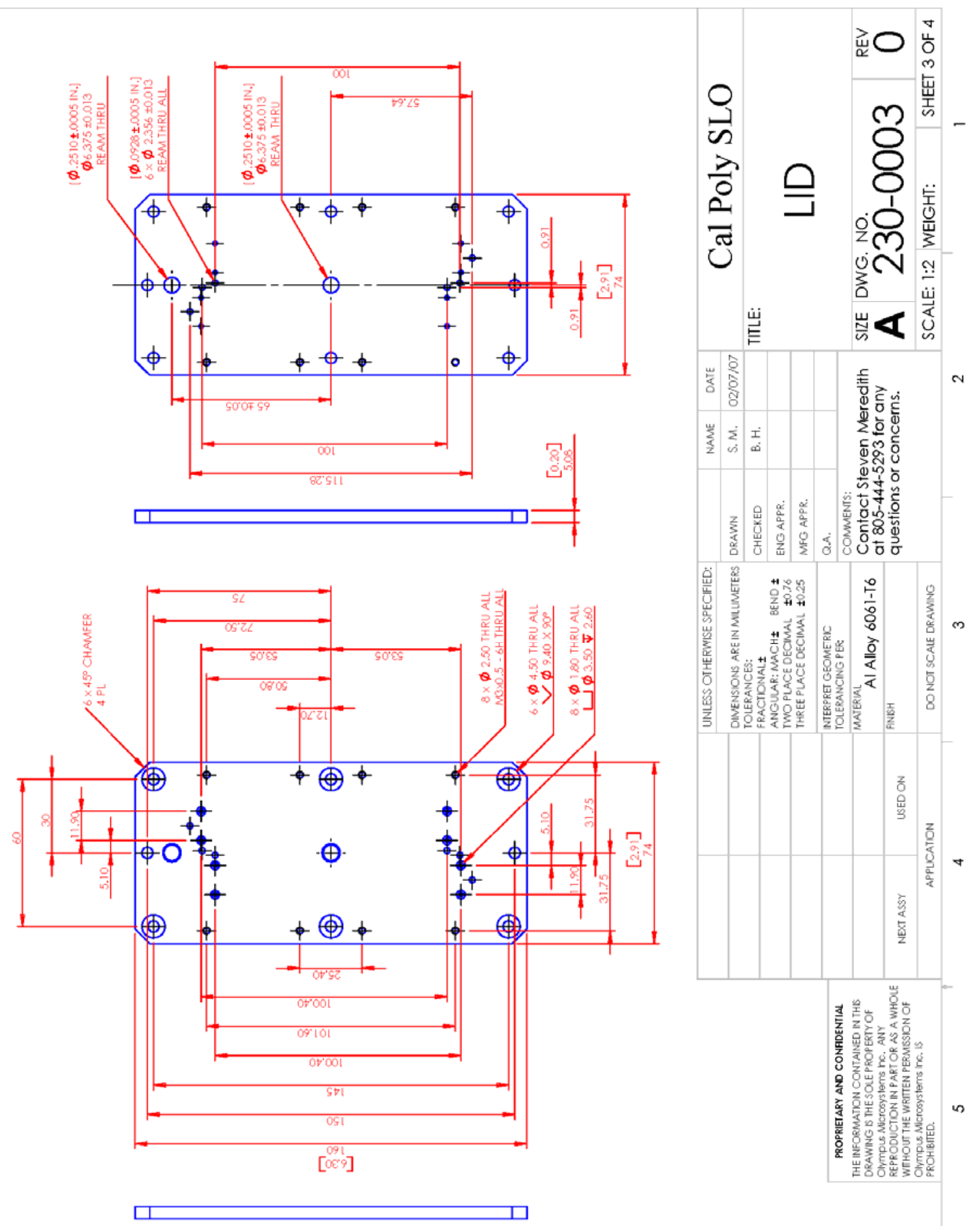

C-14 


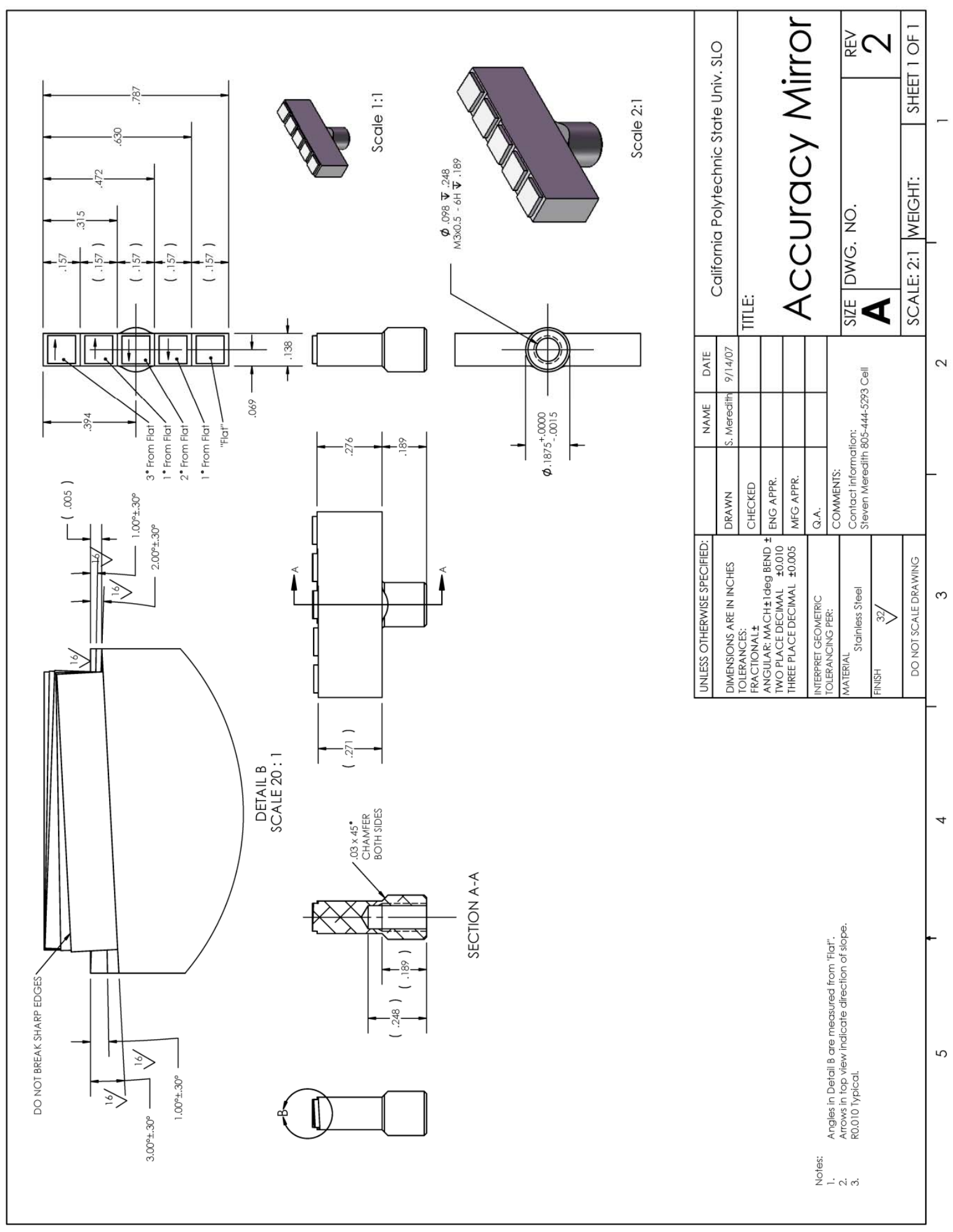

C-15 